University of Tennessee Health Science Center

UTHSC Digital Commons

$12-2007$

\title{
Neuroadaptive Changes in the Mesocortical Glutamatergic System during Nicotine Self-administration and after Extinction in Rats
}

Fan Wang

University of Tennessee Health Science Center

Follow this and additional works at: https://dc.uthsc.edu/dissertations

Part of the Medical Neurobiology Commons, Neurosciences Commons, and the Substance Abuse and Addiction Commons

\section{Recommended Citation}

Wang, Fan , "Neuroadaptive Changes in the Mesocortical Glutamatergic System during Nicotine Selfadministration and after Extinction in Rats" (2007). Theses and Dissertations (ETD). Paper 283.

http://dx.doi.org/10.21007/etd.cghs.2007.0340.

This Dissertation is brought to you for free and open access by the College of Graduate Health Sciences at UTHSC Digital Commons. It has been accepted for inclusion in Theses and Dissertations (ETD) by an authorized administrator of UTHSC Digital Commons. For more information, please contact jwelch30@uthsc.edu. 


\title{
Neuroadaptive Changes in the Mesocortical Glutamatergic System during Nicotine Self-administration and after Extinction in Rats
}

\author{
Abstract \\ The mesocorticolimbic pathway is critical in almost all aspects of drug abuse, including nicotine. Though \\ many of the neurochemical and molecular effects of nicotine have been well studied, nicotine's long-term \\ neuroadaptive effects, specifically within the mesocorticolimbic pathway, are largely undefined. Thus, in \\ current study, we determined the neuroadaptive changes in the mesocortical glutamatergic system during \\ chronic nicotine self-administration (SA), which emulates important aspects of nicotine intake by \\ humans, and after extinction. In the initial study, after 18 days of nicotine $S A$, in the medial prefrontal \\ cortex (mPFC), NMDA receptor subunit 2A (NR2A) and NR2B were increased by $67 \%$ and $83 \%$, \\ respectively. In the ventral tegmental area (VTA), glutamate receptor subunit $2 / 3$ (GluR2/3) was increased \\ by $34 \%$. These findings suggest the glutamate neurotransmission between mPFC and VTA may be \\ enhanced during chronic nicotine SA. Thus, we determined the effects of nicotine SA and extinction on \\ NMDA-induced glutamate neurotransmission between MPFC and VTA. On d 19 of ad lib access to \\ nicotine $S A$, both brain regions were microdialyzed for glutamate while $\mathrm{MPFC}$ was sequentially perfused \\ with: Kreb's Ringer Buffer (KRB), 200 mM NMDA, KRB, 500 mM NMDA, KRB, 100 mM KCl. Basal glutamate \\ levels were unaffected, but nicotine SA potentiated mPFC glutamate release to $200 \mathrm{mM}$ NMDA, which \\ was ineffective in controls. Furthermore, in VTA, nicotine SA amplified glutamate responses to both mPFC \\ infusions of NMDA. After extinction, glutamate responses were no longer enhanced, and the expression \\ of glutamate receptor subunits reverted to control levels. Behavioral studies showed that an mPFC \\ microinjection of AP-5, an NMDA-R antagonist, did not affect nicotine or sucrose SA on d 19. In contrast, \\ in VTA, NBQX, an AMPA-R antagonist, attenuated both nicotine and sucrose SA. In summary, chronic \\ nicotine SA amplified both MPFC and VTA glutamate responses to MPFC NMDA. This hyper- \\ responsiveness, along with the up-regulation of glutamate receptors, reverted to control levels after \\ extinction. Blockade of VTA AMPA-R but not MPFC NMDA-R, decreased nicotine and sucrose SA. \\ Collectively, these studies indicate that mesocortical glutamate neurotransmission adapts to chronic \\ nicotine SA and up-regulation of VTA AMPA-R may be involved in the maintenance of nicotine SA. \\ Document Type \\ Dissertation \\ Degree Name \\ Doctor of Philosophy (PhD) \\ Program \\ Pharmacology \\ Research Advisor \\ Burt M. Sharp M.D. \\ Keywords \\ medial prefrontal cortex (mPFC), ventral tegmental area (VTA), in vivo microdialysis, GABA, glutamate \\ Subject Categories \\ Medical Neurobiology | Medical Sciences | Medicine and Health Sciences | Neurosciences | Substance \\ Abuse and Addiction
}




\title{
NEUROADAPTIVE CHANGES IN THE MESOCORTICAL GLUTAMATERGIC SYSTEM DURING NICOTINE SELF-ADMINISTRATION AND AFTER EXTINCTION IN RATS
}

\author{
A Dissertation \\ Presented for \\ The Graduate Studies Council \\ The University of Tennessee \\ Health Science Center
}

\author{
In Partial Fulfillment \\ Of the Requirements for the Degree \\ Doctor of Philosophy \\ From The University of Tennessee
}

\author{
By \\ Fan Wang \\ December 2007
}


Chapter 2 @ 2007 by Nature Publishing Group. All other material @ 2007 by Fan Wang 


\section{Dedication}

This dissertation is dedicated to my parents

Mr. Yongbo Wang

And

Mrs. Jianping Li

who have given me invaluable educational opportunities.

Also this dissertation is dedicated to my wife

Yixin Liang,

for her love and support.

Without these things, this dissertation could not have been possible. 


\section{Acknowledgments}

I would like to thank Dr. Burt M. Sharp, for his guidance, patience, and giving me the opportunity to learn from him and work in his laboratory. I would also like to thank my committee members, Dr. Danniel Goldowitz, Dr. Shannon G. Matta, Dr. Jeffery D. Steketee, and Dr. Steven J. Tavalin for taking time to assist in my research. I'd like to thank Dr. Hao Chen for the help throughout my last 5 years. Finally, I would like to thank everyone who helped me during my project. 


\begin{abstract}
The mesocorticolimbic pathway is critical in almost all aspects of drug abuse, including nicotine. Though many of the neurochemical and molecular effects of nicotine have been well studied, nicotine's long-term neuroadaptive effects, specifically within the mesocorticolimbic pathway, are largely undefined. Thus, in current study, we determined the neuroadaptive changes in the mesocortical glutamatergic system during chronic nicotine self-administration (SA), which emulates important aspects of nicotine intake by humans, and after extinction. In the initial study, after 18 days of nicotine SA, in the medial prefrontal cortex (mPFC), NMDA receptor subunit 2A (NR2A) and NR2B were increased by $67 \%$ and $83 \%$, respectively. In the ventral tegmental area (VTA), glutamate receptor subunit $2 / 3$ (GluR2/3) was increased by $34 \%$. These findings suggest the glutamate neurotransmission between $\mathrm{mPFC}$ and VTA may be enhanced during chronic nicotine SA. Thus, we determined the effects of nicotine SA and extinction on NMDAinduced glutamate neurotransmission between mPFC and VTA. On d 19 of ad lib access to nicotine SA, both brain regions were microdialyzed for glutamate while $\mathrm{mPFC}$ was sequentially perfused with: Kreb's Ringer Buffer (KRB), $200 \mu \mathrm{M}$ NMDA, KRB, $500 \mu \mathrm{M}$ NMDA, KRB, $100 \mathrm{mM} \mathrm{KCl}$. Basal glutamate levels were unaffected, but nicotine SA potentiated $\mathrm{mPFC}$ glutamate release to $200 \mu \mathrm{M}$ NMDA, which was ineffective in controls. Furthermore, in VTA, nicotine SA amplified glutamate responses to both $\mathrm{mPFC}$ infusions of NMDA. After extinction, glutamate responses were no longer enhanced, and the expression of glutamate receptor subunits reverted to control levels. Behavioral studies showed that an mPFC microinjection of AP-5, an NMDA-R antagonist, did not affect nicotine or sucrose SA on d 19. In contrast, in VTA, NBQX, an AMPA-R antagonist, attenuated both nicotine and sucrose SA. In summary, chronic nicotine SA amplified both $\mathrm{mPFC}$ and VTA glutamate responses to mPFC NMDA. This hyperresponsiveness, along with the up-regulation of glutamate receptors, reverted to control levels after extinction. Blockade of VTA AMPA-R but not mPFC NMDA-R, decreased nicotine and sucrose SA. Collectively, these studies indicate that mesocortical glutamate neurotransmission adapts to chronic nicotine SA and up-regulation of VTA AMPA-R may be involved in the maintenance of nicotine SA.
\end{abstract}




\section{Table of Contents}

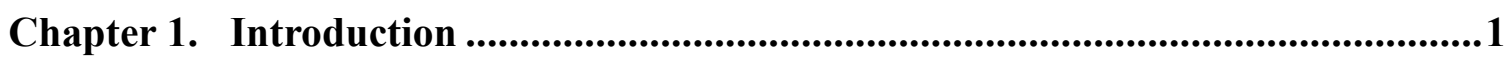

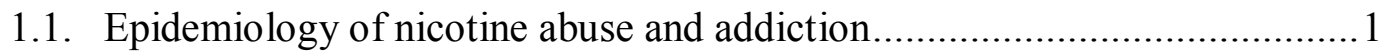

1.2. Neuropharmacology of nicotine addiction ............................................. 1

1.2.1. General mechanisms of nicotine addiction: from molecular to cellular level .....................................................................

1.2.2. Neuroanatomy of the mesocorticolimbic system and neurotransmitter and receptor systems important for nicotine

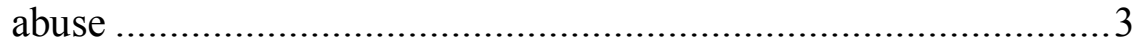

1.2.3. Neuroadaptive changes induced by nicotine SA and withdrawal.. 10

1.3. Neurotransmission in specific mesocorticolimbic regions.......................... 12

1.3.1. The functional role of glutamate system in the mPFC ..................12

1.3.2. The functional role of glutamate system in the VTA .....................13

1.3.3. The effects of the mPFC on the VTA......................................... 14

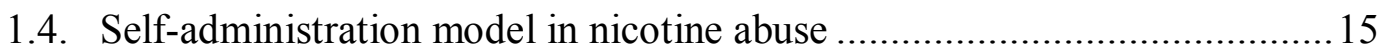

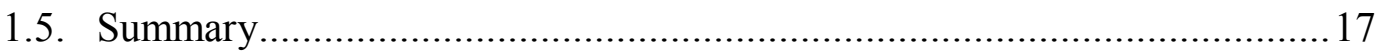

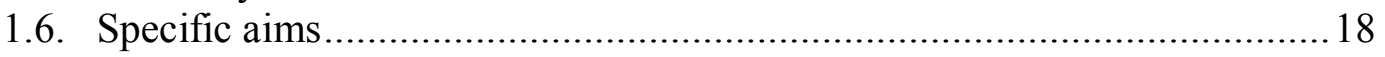

\section{Chapter 2. Selective Up-Regulation of Ionotropic Glutamate Receptor Subunits} within Mesocorticolimbic Regions during Chronic Nicotine Self-

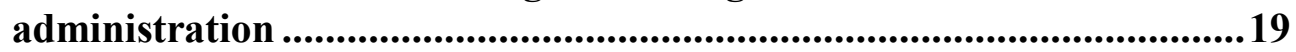

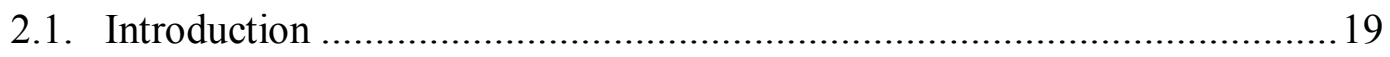

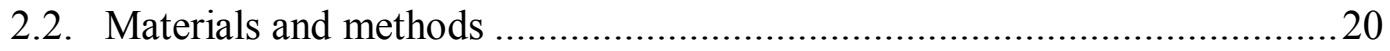

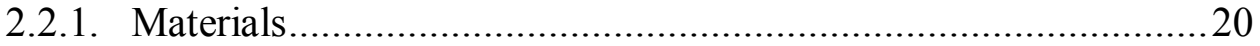

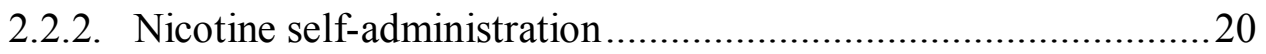

2.2.3. Brain punches and tissue preparation .......................................21

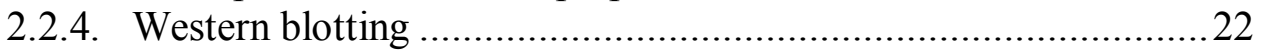

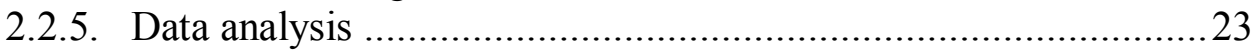

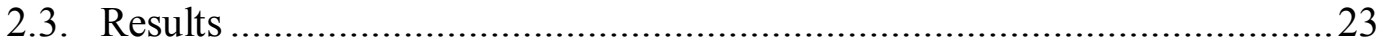

2.3.1. Nicotine self-administration...................................................23

2.3.2. Chronic nicotine self-administration-induced up-regulation of NMDA and AMPA receptor subtypes within mesocorticolimbic

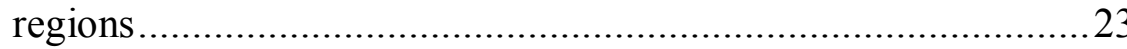

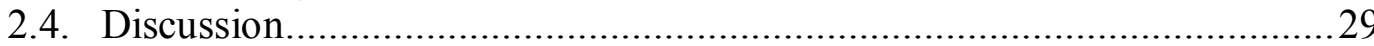

Chapter 3. Neuroadaptive Changes in the Mesocortical Glutamatergic System during Chronic Nicotine Self-Administration and after Extinction in Rats.

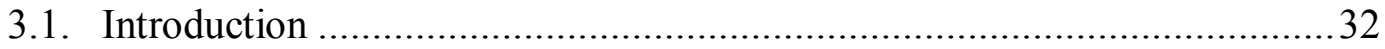

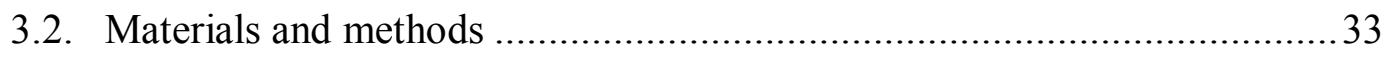

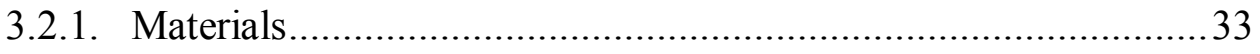

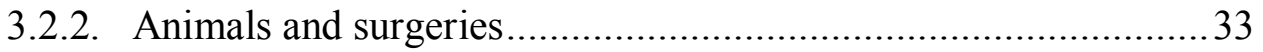

3.2.3. Procedures for chronic nicotine self-administration and extinction followed by microdialysis.............................................34 


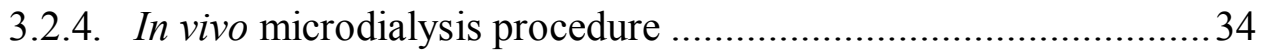

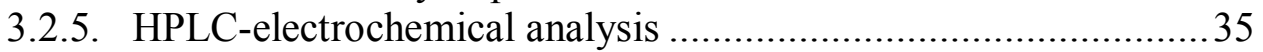

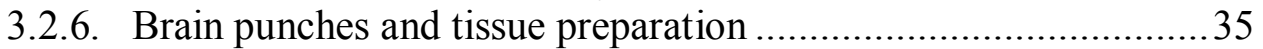

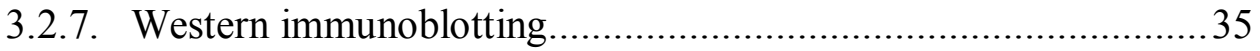

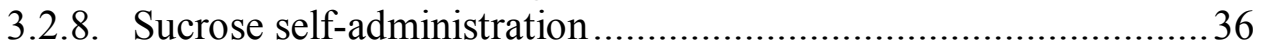

3.2.9. Intraparenchymal microinjections after chronic nicotine selfadministration or sucrose self-administration ...............................37

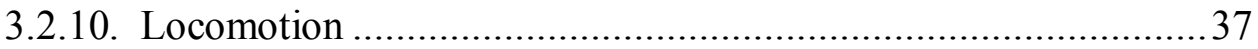

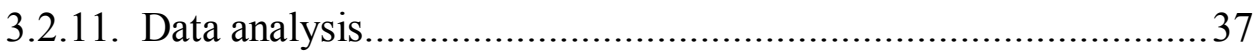

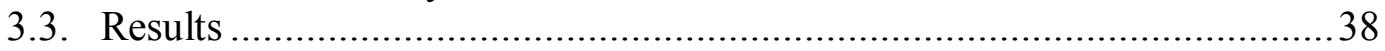

3.3.1. Chronic Nicotine SA and extinction from nicotine SA ................38

3.3.2. $\mathrm{mPFC}$ and VTA glutamate responsiveness to intra-mPFC NMDA during chronic nicotine SA............................................40

3.3.3. $\mathrm{mPFC}$ and VTA glutamate responsiveness to intra-mPFC NMDA after extinction from chronic nicotine SA...................... 40

3.3.4. $\mathrm{mPFC}$ and VTA GABA responsiveness to intra-mPFC NMDA during chronic nicotine SA …………...................................... 43

3.3.5. Expression of ionotropic glutamate receptor subunits in the mPFC and VTA after extinction from nicotine SA......................43

3.3.6. The effects of blockade of MPFC NMDA receptors on the maintenance of chronic nicotine SA and sucrose SA...................47

3.3.7. The effects of blockade of VTA AMPA receptors on the maintenance of chronic nicotine SA and sucrose SA ...................4 47

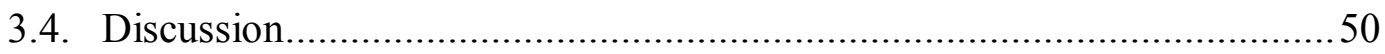

Chapter 4. Discussion ...................................................................................................54

4.1. The cellular distribution of up-regulated $\mathrm{mPFC}$ NMDA receptors and

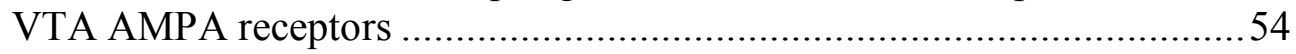

4.2. Monosynaptic and polysynaptic glutamatergic connections between

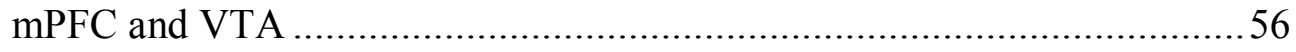

4.3. The potential functional significance of enhanced glutamate neurotransmission during chronic nicotine SA...

4.4. Enhanced glutamate neurotransmission implicated in behavioral paradigms affected by chronic administration of drugs of abuse ................58

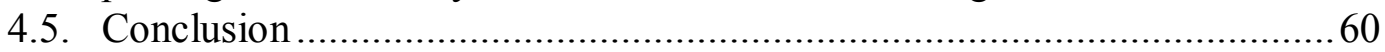

List of References ...............................................................................................................61

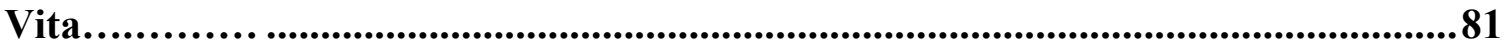




\section{List of Figures}

Figure 2.1. Lever-pressing activity during 18 days of nicotine (Nic) self-

administration compared to saline (Sal).

Figure 2.2. Nicotine self-administration (18 days) up-regulated NR2A and NR2B

subunit levels within the prefrontal cortex (PFC) of rat brain...... 25

Figure 2.3. Nicotine self-administration (18 days) increased GluR2/3 subunit immunoreactivity within the ventral tegmental area (VTA) of rat brain.....26

Figure 2.4. Nicotine self-administration (18 days) produced no statistically significant alterations in any of the subunits within the nucleus accumbens (NAcc) shell of rat brain.

Figure 2.5. Nicotine self-administration (18 days) produced no statistically significant alterations in any of the subunits within the striatum (ST) of rat brain.

Figure 3.1. Lever press activity during chronic nicotine self-administration (SA) and extinction.

Figure 3.2. Schematic representation of the positions of all microdialysis probes and microinjection cannulae in the rat $\mathrm{mPFC}$ and VTA.

Figure 3.3. Chronic nicotine SA enhanced glutamate release in the $\mathrm{mPFC}$ and VTA induced by intra-mPFC NMDA (administered by reverse microdialysis)...42

Figure 3.4. After extinction from chronic nicotine SA, glutamate release in the mPFC (panel A) and VTA (panel B), induced by intra-mPFC NMDA, was not enhanced by prior nicotine SA.

Figure 3.5. GABA responses in the $\mathrm{mPFC}$ (panel A) and VTA (panel B), induced by intra-mPFC NMDA, were not enhanced by prior nicotine SA.

Figure 3.6. After extinction of nicotine SA, the mPFC and VTA levels of the ionotropic glutamate receptor subunits, NR1, NR2A, NR2B, GluR1 and GluR2/3 were unaffected by prior nicotine SA.

Figure 3.7. The NMDA receptor antagonist, AP-5, did not affect the maintenance of chronic nicotine or sucrose SA.

Figure 3.8. The AMPA receptor antagonist, NBQX, reduced active lever pressing during the maintenance phase of chronic nicotine and sucrose SA when administered into the VTA. 


\section{Chapter 1. Introduction}

\subsection{Epidemiology of nicotine abuse and addiction}

Nicotine, an addictive substance, is the major psychoactive component in cigarette smoke. Despite the recent trend that suggests a declining prevalence of active tobacco users (Giovino et al., 1995; Hudmon et al., 2006), tobacco use in the form of cigarette smoking is still responsible for three million smoking related deaths in the United States and approximately $20 \%$ of all deaths in the western world (Smith and Fiore, 1999). According to the Centers for Disease Control and Prevention (CDC), nearly 438,000 deaths and $\$ 157$ billion in annual health-related economic losses are attributable to cigarette smoking in the United States (CDC, 2002; 2005). Worldwide, smokingrelated mortality is predicted to exceed 10 million individuals per year in the coming 30 40 years (Peto et al., 1996). All of these statistics yield one conclusion: nicotine abuse in the form of cigarette smoking is a global health epidemic. It is the leading preventable cause of morbidity and death in the United States (Koop and Luoto, 1982; Smith and Fiore, 1999).

\subsection{Neuropharmacology of nicotine addiction}

\subsubsection{General mechanisms of nicotine addiction: from molecular to cellular level}

Nicotine exerts its central and peripheral actions by binding to nicotinic acetylcholine receptors (nAChRs) that are cationic ligand-gated ion channels expressed throughout the central nervous system (CNS). Currently, twelve neuronal nAChRs subunits have been identified and cloned: $\alpha 2-\alpha 10$ and $\beta 2-\beta 4$ (Dani and Bertrand, 2007) . In contrast to the highly conserved primary structure of muscle type nAChRs (Le Novere and Changeux, 1995), the primary structures of neuronal nAChRs are highly diverse. In general, neuronal $\mathrm{nAChRs}$ can be divided into two categories: heteromeric and homomeric. $\alpha 7-\alpha 9$ subunits are capable of forming homooligomeric $\mathrm{nAChRs}$, but only $\alpha 7$ is present throughout the mammalian CNS (Dani et al., 2001). The functional properties of nAChRs vary depending on the composition of the subunits. For example, the relative permeability of calcium compared to sodium ranges from 0.1 for muscle type nAChRs to 2.0 for neuronal type heteromeric nAChRs and to greater than 10 for neuronal homomeric nAChRs (Dani and Bertrand, 2007). In addition, the potency of acetylcholine in an electrophysiological study varies as well; it has been shown that $\alpha 7$ subunit requires a higher concentration of acetylcholine for activation (EC50: approximately $200 \mu \mathrm{M}$ ) in comparison to $\alpha 4 \beta 2$ (EC50: $62 \mu \mathrm{M}$ and $1.6 \mu \mathrm{M}$ for low and high affinity component, respectively) (Buisson and Bertrand, 2001).

Distinct pharmacological profiles, dependent on subunit composition, have given rise to selective ligands. Highly selective $\alpha 7$ homomeric receptor antagonists such as $\alpha-$ 
bungarotoxin ( $\alpha$ Bgtx) (Katz and Miledi, 1973) and methyllycaconitine (MLA; at nM concentrations), which has very high affinity for the rat brain $\alpha \mathrm{Bgtx}$ binding site (Ward et al., 1990), have been discovered. Epibatidine, which binds with pM affinity to all of the receptors containing $\alpha 2, \alpha 3, \alpha 4, \alpha 6$ and $\alpha 8$ subunits, is used frequently in receptor binding studies as an agonist for heteromeric nAChRs (Gotti et al., 2000). The noncompetitive $\mathrm{nAChR}$ antagonist mecamylamine has been used widely; however, it also showed non-specific inhibition of the NMDA receptor at high micromolar concentrations. Dihydro-beta-erythroidine (DH $\beta E)$ and alpha-conotoxin-MII $(\alpha-\mathrm{CTx}-$ MII) have been identified as selective antagonists for $\alpha 4 \beta 2$ and $\alpha 3 \beta 2 / \alpha 6 \beta 2 \mathrm{nAChRs}$, respectively (Alkondon and Albuquerque, 1993; Cartier et al., 1996).

In the brain, upon binding of nicotine or endogenous acetylcholine, nAChR channels open and cations flux inward, resulting in neuronal depolarization. Depending on the location of the nAChRs, activation of nAChRs has different effects on neurons. The activation of nAChRs on post-synaptic neurons triggers some of the fast excitatory neurotransmission and many cellular processes including the modulation of cytoplasmic signal transduction affecting extracellular signal-regulated kinase (ERK) and cyclic adenosine 3',5'-phosphate (cAMP)-responsive element binding protein (CREB) (Dani and De Biasi, 2001). By using whole cell recording and optical recording techniques, fast postsynaptic currents mediated by acetylcholine were discovered first in the developing visual cortex of ferrets (Roerig et al., 1997). Under the condition of blockade of muscarinic, AMPA, NMDA, $\gamma$-aminobutyric acid receptor subtype A $\left(\mathrm{GABA}_{\mathrm{A}}\right)$, ATP, and 5-HT3 receptors, $\alpha 7$ subunit-containing nAChRs are thought to be the major nAChRs mediating the fast excitatory neurotransmission recorded in CA1 interneurons from hippocampal slice preparations (Alkondon et al., 1998; Frazier et al., 1998). The activation of $\alpha 7 \mathrm{nAChRs}$ results in a short-lasting phasic excitation of CA1 interneurons, while $\alpha 4 \beta 2 \mathrm{nAChRs}$ are thought to mediate the long-lasting tonic excitation of these interneurons (Alkondon, Pereira et al., 1999). Interestingly, $\alpha 4 \beta 2$ containinig nAchR has been shown to be involved in fast synaptic neurotransmission in the chick lateral spiriform nucleus (SpL) (Nong et al., 1999). Therefore, the excitability of neurons can be modulated by nicotinic cholinergic inputs.

The activation of presynaptic $\alpha 7$ subunit-containing nAChRs or preterminal nAChRs stimulates the release of a number of neurotransmitters by calcium influx. These include dopamine, glutamate and GABA (Dani and De Biasi, 2001). It has been reported that intravenous nicotine increases extracellular dopamine levels in the nucleus accumbens (NAcc) shell, which is thought to be a neurochemical effect common to drugs of abuse (Pontieri et al., 1996). Glutamate release was enhanced when presynaptic nAChRs on glutamatergic terminals between medial habenula nucleus (MHN) and the interpeduncular nucleus (IPN) were activated by the acute administration of nicotine (McGehee et al., 1995). Similarly, nicotine enhanced GABA release in cultured hippocampus neurons. The functional significance of interactions between nicotine and specific neurotransmitter systems is elaborated in the following section. 


\subsubsection{Neuroanatomy of the mesocorticolimbic system and neurotransmitter and receptor systems important for nicotine abuse}

\subsubsection{Neuroanatomy of the mesocorticolimbic system}

The mesocorticolimbic system is composed of the mPFC, VTA and NAcc, as well as other limbic regions including the striatum and amygdala. Pyramidal neurons in the mPFC send excitatory glutamatergic projections to the VTA and the NAcc as well as other subcortical regions (Sesack and Pickel, 1992; Taber et al., 1995). The mPFC receives dopaminergic innervations from the VTA (Thierry et al., 1973; Sesack et al., 1995). The mPFC also receives cortical glutamatergic inputs from the contralateral mPFC (Conde et al., 1995) and subcortical glutamatergic inputs from the medialdorsal thalamus (MD) (Thierry et al., 1973; Pirot et al., 1994), the CA1 region of hippocampus (Jay et al., 1989; Jay et al., 1996; Thierry et al., 2000) and the basolateral nucleus of the amygdala (BLA) (Bacon et al., 1996; McDonald, 1996). GABAergic inputs to the mPFC pyramidal neurons originate from $\mathrm{mPFC}$ GABAergic interneurons and GABA-containing neurons in the VTA (Pirot et al., 1992; Carr and Sesack, 2000).

The $\mathrm{mPFC}$ is a heterogenerous region and can be further divided into five subregions: infralimbic, dorsal and ventral anterior cingulate (areas $\mathrm{Cg} 1$ and $\mathrm{Cg} 2$, respectively), prelimbic (area Cg3) and medial precentral (Fr1) (Steketee, 2005). It has been shown that prelimbic and infralimbic cortices receive strong dopaminergic inputs from the A10 cell group of the VTA (Thierry et al., 1973; Lindvall and Bjorklund, 1978; Sesack et al., 1995). Quinolinic acid lesions of the prelimbic subregion selectively block the initiation of cocaine-induced sensitization while the same lesions of the infralimbic subregion disrupt spontaneous motor activity (Tzschentke and Schmidt, 2000). In general, the ventral mPFC projects mainly to the shell of the NAcc whereas the dorsal $\mathrm{mPFC}$ neurons impinge primarily on neurons in the core of the NAcc (Berendse et al., 1992). Furthermore, a recent tract tracing study demonstrated that the bulk of the NAcc glutamate input arises from the ventral mPFC (Gabbott et al., 2005), moreover, this same study suggested that VTA receives relatively homogenous input from the ventral and dorsal mPFC (Gabbott et al., 2005). These neuroanatomical differences extend to the functional differences between these two mPFC subregions. Functionally, lesions of the dorsal $\mathrm{mPFC}$ (dorsal prelimbic and anterior cingulate) blocked the expression of behavioral sensitization to cocaine (Pierce et al., 1998), while lesions of ventral mPFC (ventral prelimbic and infralimbic) failed to do so (Tzschentke and Schmidt, 1999).

Traditionally, the VTA is thought to be composed of two types of neurons: GABAergic and dopaminergic neurons (Johnson and North, 1992). VTA dopamine neurons project to the NAcc and the mPFC. VTA GABAergic neurons project to the mPFC (Carr and Sesack, 2000) and the NAcc (Van Bockstaele and Pickel, 1995), while GABAergic interneurons synapse primarily on VTA DA neurons (Steketee, 2005). Traditionally, the VTA is thought to receive excitatory inputs primarily from the mPFC (Hurley et al., 1991), however, a recent paper provided new evidence that subcortical 
regions (i.e., laterodorsal tegmental nucleus) are also important sources of glutamate to the VTA (Omelchenko and Sesack, 2007). Moreover, mPFC glutamatergic projections to the VTA seem to be target-specific: mPFC glutamatergic inputs selectively synapse on mesoaccumbens GABAergic neurons in the VTA and on mesocortical dopaminergic neurons (Carr and Sesack, 2000). Furthermore, a recent study showed that primary glutamatergic neurons also exist in the VTA as evidenced by the presence of vesicular glutamate transporter 2 (VGluT2) mRNA without co-expression of markers characteristic of dopaminergic or GABAergic neurons (Yamaguchi et al., 2007). However, the functional role of these intrinsic VTA glutamatergic neurons is unclear.

The mesolimbic dopaminergic pathway is thought to be a fundamental anatomical substrate that mediates natural reward and the reinforcing effects of drugs of abuse (Wise, 1996; Wise, 2006). The NAcc receives dopaminergic and GABAergic inputs from the VTA and receives glutamatergic inputs from the mPFC (Sesack and Pickel, 1992). The NAcc also sends GABAergic projections back to the VTA. Thus, the NAcc is regulated by the mPFC as well as the VTA. In the preceding sections, we have discussed the different functional roles of the core vs. shell of the NAcc as well as the preferential innervation from $\mathrm{mPFC}$ subregions (Groenewegen et al., 1990). Though this dissertation focuses on the mPFC-VTA glutamatergic circuitry, this pathway must be understood in the context of other neuroanatomical regions, regulatory circuits and neurotransmitters within the mesocorticolimbic system (e.g. NAcc and inputs from other brain nuclei such as serotonergics from dorsal raphe, and noadrenergics from locus coeruleus (LC), which will be discussed extensively in the Discussion chapter).

\subsubsection{Dopaminergic system}

The dopamine hypothesis postulates that the mesolimbic dopaminergic system has an important role in transducing the signals for reinforcement that are triggered by natural rewards and drugs of abuses including nicotine (Spanagel and Weiss, 1999). It is supported by the pivotal finding that drugs (e.g., nicotine, opiates, ethanol, amphetamine, cocaine) increased extracellular dopamine levels in the NAcc of freely moving rats (Di Chiara and Imperato, 1988). As mentioned above, nicotine is able to induce neurotransmitter release by binding to and activating nAChRs. In situ hybridization experiments have demonstrated that mRNAs for a majority of the neuronal nAChR subunits (e.g., $\alpha 2, \alpha 3, \alpha 4, \alpha 5, \alpha 6, \alpha 7, \beta 2$ and $\beta 3$ ) are expressed in mesolimbic dopamine regions (Di Chiara, 2000). Furthermore, $\beta 2, \alpha 6$ and $\alpha 4$ subunits were found on dopamine neurons in the VTA by immunohistochemistry (Di Chiara, 2000). These findings support the idea that nicotine is capable of modulating dopamine neurotransmission.

Several lines of evidence have demonstrated that the dopaminergic system is crucial for nicotine self-administration. The microinjection of 6- hydroxydopamine (6OHDA) into the NAcc, which selectively lesioned the mesolimbic dopamine system, significantly reduced nicotine self-administration (SA) (Corrigall et al., 1992).

Furthermore, it has been demonstrated that systemic administration of both a selective D1 receptor antagonist (SCH23390) and a D2 receptor antagonist (spiperone) were able to 
attenuate nicotine SA in a dose-dependent manner (Corrigall and Coen, 1991). Collectively, these experiments indicate that the mesolimbic dopamine system has a crucial role in mediating the reinforcing effects of nicotine.

Conditioned place preference is another animal model to study the reinforcing effects of drugs of abuse. It is based on the observations that animals prefer the environment that is paired with a positive motivational stimulus. The effects of nicotine on conditioned place preference remain controversial. Both place aversion and preference were reported when different doses of nicotine were directly infused into the VTA (Laviolette and van der Kooy, 2003). Interestingly, both systemic and intra-accumbens administration of the dopamine receptor antagonist, $\alpha$ - flupenthixol, were able to potentiate nicotine place preference (Laviolette and van der Kooy, 2003). These observations suggest that mesolimbic dopamine signaling might mediate some of the aversive properties of nicotine, and thus blockade of mesolimbic dopamine signaling may enhance the reinforcing effects of nicotine (Laviolette and van der Kooy, 2003).

D1 and D2 receptors have been shown to be involved in the reinforcing effects of nicotine. For example, systemic administration of selective D1 (SCH23390) or D2 (spiperone) dopamine antagonists attenuated the maintenance of nicotine SA (Corrigall and Coen, 1991). The D3 receptor localizes predominantly in limbic areas, and a subpopulation appears to function as autoreceptors that modulate dopamine release (Heidbreder et al., 2005). However, less is known regarding to the roles of D3 receptor subtypes in nicotine addiction, largely due to the lack until recently of a selective D3 receptor antagonist. In 2003, the effects of a selective D3 receptor antagonist, SB277011-A (100 fold selectivity for D3 receptor subtype over D2), on the maintenance of nicotine SA and on nicotine induced nicotine reinstatement were reported (Andreoli et al., 2003). SB-277011-A (3-10 mg/kg, i.p.) substantially reduced nicotine priminginduced reinstatement, while it had no effect on the maintenance of nicotine SA (Andreoli et al., 2003). This suggests that different dopamine receptor subtypes might have distinct roles in nicotine abuse and addiction.

At the concentrations of nicotine found in the blood of smokers (Henningfield et al., 1993), nicotine depolarizes the VTA dopamine neurons in a rat midbrain slice preparation and then desensitizes within seconds (Pidoplichko et al., 1997). However, enhanced dopamine release can last for more than one hour, as shown by in vivo microdialysis (Imperato et al., 1986; Di Chiara and Imperato, 1988). This discrepancy suggests that additional mechanisms may contribute to the sustained dopamine release induced by transient nAChR activation.

\subsubsection{Glutamatergic system}

The glutamate system is the primary excitatory neurotransmitter system in the CNS and has been shown to regulate the mesolimbic dopamine system -specifically the A10 dopamine neurons in the VTA (Kalivas et al., 1989). Nicotine has also been shown to enhance glutamate release in a number of brain regions across multiple species, 
probably through presynaptic $\alpha 7$ subunit-containing nAChRs (Mansvelder and McGehee, 2000). Systemic nicotine $(0.5 \mathrm{mg} / \mathrm{kg}$, s.c. $)$ significantly increased extracellular levels of both aspartate and glutamate measured by in vivo microdialysis in the VTA (Schilstrom et al., 2000). This enhanced glutamate release was blocked by intra-tegmental infusion of the $\alpha 7 \mathrm{nAChR}$ antagonist methyllycaconitine (MLA; 0.3mM) (Schilstrom et al., 2000). In an electrophysiology study, the $\alpha 7 \mathrm{nAChR}$ antagonist, $\alpha-\mathrm{BgTx}$, blocked the nAChRmediated spontaneous postsynaptic glutamate currents (PSC) in the chick ventral lateral geniculate nucleus (Guo et al., 1998). Consistent with this study, it has been demonstrated that local application of nicotine into the VTA enhanced glutamatergic inputs (spontaneous EPSCs), which led to long-term potentiation (LTP) in a $\alpha 7$ receptordependent manner (McGehee et al., 1995; Mansvelder and McGehee, 2000). Similarly, local application of nicotine enhanced glutamate release from the thalamocortical terminals in a preparation of prefrontal cortex (Lambe et al., 2003).

A large number of reports have examined the functional significance of nicotineenhanced glutamate input to dopamine neurons. Several lines of evidences have suggested that nicotine may modulate DA release by influencing presynaptic nAChRs on glutamatergic inputs to dopamine neurons. In an in vivo microdialysis study (Schilstrom et al., 1998), the enhanced dopamine release in the NAcc after systemic nicotine injection was reduced by intra- tegmental perfusion of 2- amino- 5- phosphonopentanoic acid (AP5) but not by 6- cyano- 7- nitroquinoxaline- 2,3- dione (CNQX), suggesting a pivotal role of VTA NMDA receptors in mediating the reinforcing effects of nicotine. Furthermore, our laboratory has demonstrated that the increased dopamine release induced in the NAcc by a low dose of nicotine (i.v. $0.065 \mathrm{mg} / \mathrm{kg}$ ) or a higher dose $(0.09 \mathrm{mg} / \mathrm{kg}$ ) was abolished or reduced by intra-tegmental administration of AP-5 (Fu et al., 2000). Moreover, the level of VTA glutamate was only affected by higher doses of nicotine, indicating that the enhancement of NAcc DA release by lower doses of nicotine involves the tonic activation of VTA NMDA receptors (Fu et al., 2000). In line with these studies, systemic administration of another NMDA receptor antagonist, MK-801 (dizocilpine), also blocked nicotine-induced dopamine release in the NAcc (Sziraki, Sershen et al., 1998). Surprisingly, systemic administration of the NR2B receptor antagonist, Ro 25-6981 (10 $\mathrm{mg} / \mathrm{kg}$ i.p.), significantly potentiated nicotine-induced DA release in the NAcc, as well as nicotine-induced locomotor activity (Kosowski and Liljequist, 2004). It is difficult to reconcile this data that was obtained using a selective NMDA antagonist administered systemically. Taken together, these reports suggest that NMDA receptors in the VTA are pivotal in modulating nicotine-induced DA release in the NAcc, while studies using receptor subtype selective antagonists or agonists might reveal the role of specific NMDA receptor subtypes in nicotine-induced DA release.

There have been few studies to evaluate the role of the glutamate system in animal models of nicotine reinforcement. One study demonstrated that an NMDA receptor channel blocker, memantine, prevented acquisition of nicotine SA in mice (Blokhina et al., 2005). However, memantine is not a selective NMDA receptor antagonist, affecting other receptors as well, e.g., 5-HT3 receptor and nAChRs with similar or lower potency (Blokhina et al., 2005). Thus, its potential inhibitory effects on 
the nicotinic receptor channel needs to be taken into consideration to interpret the data accurately.

Metabotropic glutamate receptors (mGluR) are one of the G-protein coupled receptors (GPCR) that have been shown to regulate neurotransmitter release by modulating the activity of calcium and potassium channels (Cartmell and Schoepp, 2000). The mGluR5 receptor antagonist, MPEP, has been reported to reduce nicotine SA in rats and in mice (Paterson et al., 2003) and to diminish nicotine-seeking behavior induced by nicotine priming (Tessari et al., 2004), possibly by modulating mesolimbic dopamine neurotransmission. In a follow-up experiment, increased glutamate neurotransmission, induced by the inhibition of presynaptic inhibitory mGluR $2 / 3$ autoreceptors, was shown to diminish the effect of postsynaptic excitatory mGluR5 receptor blockade on nicotine SA (Liechti and Markou, 2007). Thus, metabotropic glutamate receptors are implicated in nicotine reinforcement.

In summary, nicotine increases glutamate release by binding to presynaptic $\alpha 7$ receptors on glutamatergic terminals in mesocorticolimbic regions, including the VTA, NAcc and PFC (Mansvelder and McGehee, 2000). Neuroanatomically, subcortical glutamatergic inputs to VTA innervate the mesoaccumbens DA neurons (Omelchenko and Sesack, 2007). Thus, NMDA receptors in the VTA, probably those expressed by mesoaccumbens DA neurons, are pivotal in regulating DA release in the NAcc (Schilstrom et al., 1998); however, only tonic activation of NMDA receptors in the VTA was involved under low dose nicotine administration (Fu et al., 2000). The acquisition of nicotine SA in mice was shown to be blocked by NMDA receptor channel blocker memantine, though its nonspecific effects on other receptors limits the interpretation of this data (Blokhina et al., 2005). Blockade of postsynaptic excitatory mGluR5 receptors reduces nicotine SA in rats and mice (Paterson et al., 2003), which can be antagonized by inhibiting presynaptic inhibitory mGluR2/3 receptors (Liechti and Markou, 2007). Collectively, these observations implicate both ionotropic and metabotropic glutamate receptors in mediating the reinforcing effects of nicotine, probably by regulating the activity of VTA dopamine neurons.

\subsubsection{Cholinergic and other systems}

It has been proposed that brainstem cholinergic, GABAergic, noradrenergic and serotonergic nuclei may be involved in mediating the reinforcing effects of nicotine (Picciotto and Corrigall, 2002). The VTA dopamine neurons receive cholinergic inputs from the caudal pedunculopontine tegmental nucleus (PPTg) and the adjacent laterodorsal tegmental nucleus (LDTg) (Oakman et al., 1995). Electrical stimulation of the laterodorsal tegmentum (LDT) evoked a three-component change in the NAcc DA efflux, characterized by a initial increase with a mean peak current of $+0.22 \pm 0.05 \mathrm{nA}$ followed by a decrease in the DA signal with a mean maximal current of -0.23 $\pm 0.05 \mathrm{nA}$, and a third prolonged enhancement of DA efflux with a mean peak current of $+0.88 \pm 0.05 \mathrm{nA}$ (Forster and Blaha, 2000). Both a intra-VTA microinjection of nAChR antagonist, mecamylamine $(5 \mu \mathrm{g} / 0.5 \mu \mathrm{L})$, and ionotropic glutamate receptor (iGluR) 
antagonist, kynurenate $(10 \mu \mathrm{g} / \mu \mathrm{L})$, attenuated the first facilitatory component by $77 \%$ and $59 \%$, respectively, while sparing the second and the third components, indicating the initial increase of DA efflux involves cholinergic and glutamatergic inputs to VTA DA neurons from LDT and the nAChRs and iGluRs in the VTA (Forster and Blaha, 2000). Intra-LDT infusion of either a nonselective muscarinic or a selective $\mathrm{M} 2$ receptor antagonists, scopolamine $(100 \mu \mathrm{g} / \mu \mathrm{L})$ or methoctramine $(50 \mu \mathrm{g} / \mu \mathrm{L})$, respectively, abolished the second component of NAcc dopamine release evoked by electrical stimulation of LDT. These muscarinic antagonists inhibited the muscarinic M2 autoreceptors on LDT cholinergic neuron cell bodies, reducing the negative cholinergic feedback (Forster and Blaha, 2000). The third component of DA efflux was reduced by an intra-VTA microinjection of the muscarinic antagonist, scopolamine $(200 \mu \mathrm{g} / \mu \mathrm{L})$ (Forster and Blaha, 2000). Collectively, these data support the notion that mesolimbic dopaminergic neurons are regulated by both cholinergic and glutamatergic projections from LDT to VTA. Both nAChRs and muscarinic cholinergic receptors in the VTA mediate the effects of this cholinergic input.

Systemic nicotine ( 0.3 or $1.0 \mathrm{mg} / \mathrm{kg}$, s.c.) has been shown to markedly increase cFos immunoreactivity in both PPTg and LDTg (Jose Lanca et al., 2000). To evaluate the role of cholinergic neurons in PPTg in the maintenance of nicotine SA, ethylcholine mustard aziridinium ion, a selective cholinergic toxin, was used to lesion the PPTg cholinergic neurons (Lanca et al., 2000). Histological examination revealed an approximate $70 \%$ reduction in NADPH-d positive neurons, which signify cholinergic neurons in the PPTg (Lanca et al., 2000), while non-cholinergic neurons in the PPTg were largely spared; this is indicative of a selective cholinergic lesion (Lanca et al., 2000). This lesion was found to reduce nicotine SA by $40 \%$ after rats acquired stable operant responding (Lanca et al., 2000). These experiments indicate that cholinergic neurons in the PPTg as well as the LDTg play a role in nicotine SA by regulating VTA dopamine neuronal activity.

It is known that VTA dopamine neurons receive GABAergic inputs not only from local GABA interneurons but also from descending GABAergic inputs originating in the ventral pallidum and NAcc (Walaas and Fonnum, 1979; Sugita et al., 1992). These inhibitory inputs directly regulate the activity of VTA dopamine neurons, indirectly affecting somatodendritic dopamine release and dopamine neuronal activity (Klitenick et al., 1992). The inhibition of dopaminergic tone in the VTA by the $\mathrm{GABA}_{\mathrm{A}}$ receptor agonist muscimol or $\mathrm{GABA}_{\mathrm{B}}$ receptor agonist baclofen has been shown to reduce the $\mathrm{DA}$ release in the NAcc (Westerink et al., 1996). Gamma vinyl-GABA (GVG), which increases GABA release, dose-dependently inhibited nicotine-induced dopamine release in the NAcc; 100 and $150 \mathrm{mg} / \mathrm{kg} \mathrm{GVG}$ both abolished nicotine-induced dopamine release (Dewey et al., 1999). A dose of $18.75 \mathrm{mg} / \mathrm{kg} \mathrm{GVG}$ completely blocked the expression of nicotine induced CPP, and $75 \mathrm{mg} / \mathrm{kg}$ dose abolished the acquisition of nicotine-induced CPP (Dewey et al., 1999). Furthermore, GVG has been shown to reduce nicotine SA by increasing GABA neurotransmission (Paterson and Markou, 2002). Somatodendritic VTA dopamine regulates VTA DA neurons via tonic auto-inhibition, mediated by D2 auto-receptors located on the VTA DA neurons. D2 receptor agonists reduce the firing rate of dopamine neurons and inhibit DA release in the VTA, while D2 receptor 
antagonists increase somatodendritic DA release in the VTA (Adell and Artigas, 2004). Thus, the regulation of somatodendritic DA release is another mechanism whereby, the GABA system is involved in regulating VTA dopamine neuronal activity.

The $\mathrm{GABA}_{\mathrm{B}}$ receptor has recently received greater attention than the $\mathrm{GABA}_{\mathrm{A}}$ receptor due to the more selective expression of the VTA GABA $A_{B}$ receptor and its effects on the reinforcing properties of drugs of abuse. These two receptors are differentially distributed in that $\mathrm{GABA}_{\mathrm{B}}$ receptors are largely expressed on the cell body of dopamine neurons (Kalivas et al., 1993) while $\mathrm{GABA}_{\mathrm{A}}$ receptors are expressed on GABA interneurons and the cell body of dopamine neurons (Klitenick et al., 1992). Indeed, intra-tegmental infusion of the $\mathrm{GABA}_{\mathrm{B}}$ receptor agonists, baclofen, significantly reduced nicotine SA (Corrigall et al., 2000). Acute systemic administration of the GABA agonists, baclofen (Fattore et al., 2002; Paterson et al., 2004) and CGP44532 (Paterson et al., 2004; Paterson et al., 2005) also reduced nicotine SA and cue- induced nicotine reinstatement, respectively. Moreover, repeated systemic administration of CGP44532 also decreased nicotine SA (Paterson et al., 2005). Taken together, these findings support the hypothesis that GABAergic mechanisms may influence the reinforcing effects of nicotine by modulating dopamine neuronal activity.

Nicotine is known to stimulate NE release in various brain regions including cortex (Summers and Giacobini, 1995), hypothalamic paraventricular nucleus (PVN) (Fu et al., 2001) and amygdala (AMG) (Fu et al., 2003). The studies from our laboratory showed a positive correlation between the operant nicotine SA behavior and the increment of NE release in the PVN and the AMG (Fu et al., 2001; Fu et al., 2003). Additionally, nAChRs in the brainstem nucleus tractus solitarius (NTS) along with the NMDA receptor and nitric oxide (NO) mediate the stimulatory effects of nicotine on NE release in the PVN and the AMG (Fu et al., 1997; Zhao et al., 2007). Although few studies provide a direct link between the NE system and the reinforcing effects of nicotine, systemic administration of $\alpha 2$ adrenergic agonists, at doses shown to block footshock-induced release of NE in the PFC and in the AMG, prevented the footshockinduced reinstatement of cocaine seeking but not cocaine-priming induced reinstatement. This suggests that the NE system may play a role in stress-induced reinstatement, perhaps through amygdaloid mechanisms (Erb et al., 2000; Kalivas and McFarland, 2003).

Studies have suggested that serotonin (5-HT) may be involved in the reinforcing effects of nicotine. A low dose of nicotine $(1 \mu \mathrm{M})$ was perfused locally via a microdialysis probe into several brain areas, resulting in a decrease in 5-HT levels in the hippocampus (Hippo) and the VTA, and an increase in 5-HT levels in the AMG (Shearman et al., 2005). Chronic nicotine administration ( 3 or $12 \mathrm{mg} / \mathrm{kg}$ /day) for 21 days increased tissue 5-HT levels in the frontal cortex and decreased levels in the hypothalamus (Kirch et al., 1987). Prenatal nicotine exposure was implicated in changing serotoninergic neurotransmission, which was evident during adolescence (Slotkin et al., 2006). Although 5-HT3 and 5-HT1A receptors do not seem to be involved in nicotine SA, as shown by the selective 5-HT3 antagonists, ICS205-930 and MDL 72222, and the 5-HT1A agonist, ipsapirone (Corrigall and Coen, 1994; Mosner et al., 1997), the 5-HT2C receptor antagonist, RO 60-0178, attenuated the maintenance of nicotine SA in rats (Grottick et 
al., 2001), indicating that specific serotonergic receptor subtypes might be involved in nicotine SA.

Several lines of evidence indicate that the opioid system might be involved in nicotine SA. First, a high density of $\mu$ opioid receptors has been identified in the NAcc (Tempel and Zukin, 1987). Second, systemic nicotine has been shown to elevate tissue levels of opioid peptides in the NAcc (Pierzchala et al., 1987; Houdi et al., 1991). Third, nicotine can release endogeneous opioid peptides (Davenport et al., 1990). The results obtained from a series of neuropharmacological studies revealed the differential involvement of opioid receptors in a region-specific manner. Naltrexone $(0.1-10 \mathrm{mg} / \mathrm{kg}$, s.c.), administered 20 min prior to the start of nicotine SA, had no effect on nicotine SA (Corrigall and Coen, 1991). However, at the highest dose (dose range: 0.005-0.05 $\mu \mathrm{g}$ ), an intra-tegmental infusion of the selective $\mu$ opioid receptor agonist, DAMGO, affected nicotine SA. Additionally, an intra- PPTg infusion of DAMGO reduced nicotine SA (Corrigall et al., 2002).

\subsubsection{Neuroadaptive changes induced by nicotine $\mathrm{SA}$ and withdrawal}

Nicotine activates and desensitizes nAChRs rapidly (Mansvelder and McGehee, 2000), but enhanced DA release by nicotine is sustained for up to two hours (Imperato et al., 1986; Di Chiara and Imperato, 1988). Following extinction, three forms of reinstatement have been described: cue-induced, stress-induced and drug-priming injection-induced reinstatement (Shaham et al., 2003). After extinction of nicotine seeking behavior, presentation of nicotine-associated stimuli (e.g. cue light) or the delivery of a stressor (e.g., intermittent footshock) is able to reinstate nicotine seeking behavior (Buczek et al., 1999; Liu et al., 2006). Prolonged neuroadaptive changes may underlie the relapse to drug seeking behavior. For example, the up-regulation of the cAMP pathway in the NAcc represents one key neuroadaptive change (Self and Nestler, 1998). Interestingly, it has been shown that modulating iGluR receptor expression facilitates extinction, thus reducing the risk of relapse. Viral-mediated overexpression of GluR1 and GluR2/3 receptors in the NAcc shell, which have been shown to be upregulated after extinction training, facilitates the extinction of cocaine seeking behavior (Sutton et al., 2003). Furthermore, disruption of actin cycling has been shown to facilitate cocaine-induced reinstatement of cocaine seeking behavior, indicating that neuroplastic changes in actin cycling might contribute to reinstatement of drug seeking behavior (Toda et al., 2006). In summary, multiple neuroadaptive changes might contribute to the long-term effects of nicotine and other drugs of abuse, thereby predisposing to relapse.

Approximately $70 \%$ of adult smokers in the United States have tried to quit, but these attempts are often unsuccessful due to the aversive aspects of the nicotine withdrawal syndrome (Kenny and Markou, 2001). Experiments found that withdrawal from chronic nicotine gradually reduced the nicotine-induced enhancement of NMDA responsiveness in the $\mathrm{CA} 1$ region of hippocampus, which returned to baseline by day 8 of withdrawal. This may underlie the cellular mechanism(s) of nicotine withdrawal (Yamazaki et al., 2006). In addition, up-regulation of $\alpha 7$ receptors (the chronic nicotine 
control group did not show up-regulation) and reduced GABA release in the striatum was observed in this withdrawal study, indicating neuroplastic changes in the glutamatergic, cholinergic and GABAergic systems are involved during nicotine withdrawal (Yamazaki et al., 2006). Taken together, these findings indicate that nicotine may induce neuroadaptive changes during the exposure to nicotine and after withdrawal.

Nicotine has been shown to facilitate long term potentiation (LTP) of glutamatergic inputs in the VTA (Mansvelder and McGehee, 2000). It is hypothesized that $\alpha 7 \mathrm{nAChRs}$ and NMDA receptors have complementary roles in synaptic plasticity because both receptors are highly permeable to $\mathrm{Ca}^{2+}$ (Broide and Leslie, 1999). Activation of presynaptic $\alpha 7$ nAchRs by nicotine in the VTA induces the influx of $\mathrm{Ca}^{2+}$, which in turn increases glutamate release. The increased glutamate release facilitates activation of postsynaptic NMDA and non-NMDA receptors, which depolarize DA neurons and contribute to the induction of LTP (Mansvelder and McGehee, 2000). In addition, activation of postsynaptic nAChRs by nicotine also contributes to the induction of LTP by removing $\mathrm{Mg}^{2+}$ blockade of the postsynaptic NMDA receptors (Pidoplichko et al., 1997). In this study, which utilized the concentration of nicotine present in a smokers' blood after one cigarette, nicotine $(0.5 \mu \mathrm{M})$ increased the spontaneous EPSC frequency by approximately 2 fold in 90\% of the neurons tested (Mansvelder and McGehee, 2000), and this effect was blocked by selective $\alpha 7$ antagonist MLA, indicating a predominant presynaptic mechanism underlying increased glutamate release by nicotine (Mansvelder and McGehee, 2000). Moreover, pairing nicotine ( $1 \mu \mathrm{M})$ with postsynaptic depolarization (200 stimulations at a frequency of $1 \mathrm{~Hz}$ ) increased the evoked EPSC amplitude to $116 \% \pm 2.3 \%$ of control for at least $40 \mathrm{~min}$ (Mansvelder and McGehee, 2000). Although the magnitude of this LTP was quite modest, the onset and duration were consistent with LTP. This is the first paper to implicate nicotine in the induction of long-term changes after a brief exposure.

Nicotine is also known to influence the structure of neurons (Robinson and Kolb, 2004). Repeated nicotine injections $(0.7 \mathrm{mg} / \mathrm{kg})$ increased dendritic length and spine density in the NAcc and the cingulate cortex (Brown and Kolb, 2001). This appears to be a phenomenon common to other drugs of abuse, including cocaine, amphetamine and morphine (Robinson and Kolb, 2004). Moreover, nicotine stimulated dendritic arborization in the motor cortex (Gonzalez et al., 2005). However, since these experiments were preformed under the condition of experimenter-administered (EA) nicotine, the outcomes might be different with nicotine SA (Robinson and Kolb, 2004).

There have been very few papers that have examined neuroadaptation after nicotine SA and withdrawal. Our lab has shown that nAChRs and ionotropic glutamate receptors are altered by nicotine SA (Parker et al., 2004; Wang et al., 2007). During nicotine SA, neuronal nAChRs in several brain regions were up-regulated, specifically, $\alpha 4, \alpha 6$ and $\beta 2$ (Parker et al., 2004). The up-regulation $\alpha 4 \beta 2$ by nicotine has been reviewed (Buisson and Bertrand, 2002). This upregulation of surface $\alpha 4 \beta 2 \mathrm{nAChRs}$ has been shown to reflect an increased rate of intracellular maturation (Sallette et al., 2005). Under similar experimental conditions, after 18 days of nicotine SA, we have shown that the levels of NMDA receptor subunit 2A (NR2A) and NR2B were increased by $67 \%$ and 
$83 \%$ in the $\mathrm{mPFC}$, respectively, and glutamate receptor subunit $2 / 3$ (GluR2/3) was increased by $34 \%$ in the VTA (Wang et al., 2007). However, the expression of ionotropic glutamate receptors was not changed in the striatum (ST) and in the NAcc, indicating a region-specific effect of nicotine SA (Wang, Chen et al., 2007). One study of dopamine neurotransmission after early abstinence from limited access chronic nicotine SA (25 days) observed reduced basal dopamine levels in the nicotine treatment groups (both nicotine SA and yoked nicotine group) compared to saline (Rahman et al., 2004). This was due in part to increased DA reuptake after chronic nicotine treatment. The reduced elevation of extracellular dopamine levels in the NAcc after nicotine injection indicated that $\mathrm{nAChR}$ desensitization may occur early in abstinence after chronic nicotine (Rahman et al., 2004). Overall, these observations provide clear evidence that neuroadaptive changes take place during nicotine SA and withdrawal.

\subsection{Neurotransmission in specific mesocorticolimbic regions}

\subsubsection{The functional role of glutamate system in the $\mathrm{mPFC}$}

Pyramidal neurons send glutamatergic projections to many subcortical regions including the NAcc and the VTA. They also receive glutamatergic inputs as well as GABAergic and dopaminergic inputs. Thus, the mPFC pyramidal neurons are highly regulated. Many laboratories have reported that $\mathrm{MPFC}$ is critical in regulating various behaviors during different phases of drug SA. These include the sensitization resulting from repeated psychostimulant treatment (Wolf et al., 1995; Li and Wolf, 1997; Li et al., 1999; Li et al., 1999) and reinstatement of cocaine seeking behavior (McFarland et al., 2003). Additionally, mPFC contributes to general physiological responses such as to restraint stress (Moghaddam, 1993; Jedema and Moghaddam, 1994).

Several studies have implicated mPFC glutamate in the development of sensitization to psychostimulants. Ibotenic acid lesions of the PFC prevented the development of sensitization of ambulatory hyperactivity, but not the sensitization of stereotypy, after six repeated daily amphetamine treatments (Wolf et al., 1995). However, this study did not differentiate the effects of these lesions on the acquisition vs. expression of locomotor sensitization. In another study (Li and Wolf, 1997), lesioning the PFC after the induction of sensitization failed to prevent the expression of sensitization to amphetamine (Li and Wolf, 1997). A similar PFC lesion also prevented the induction of cocaine sensitization ( $\mathrm{Li}$ et al., 1999), but was unable to block the expression of cocaine sensitization (Li et al., 1999). However, a specific lesion of dorsal mPFC, and not ventral mPFC prevented expression of cocaine sensitization (Pierce et al., 1998). Consistent with the idea that ventral and dorsal mPFC might have different roles in cocaine induced behavioral sensitization, it has been found that excitotoxic lesions of prelimbic $\mathrm{mPFC}$ regions, which provide dense projections to the VTA, blocked the induction of cocaine sensitization (Tzschentke and Schmidt, 2000; Tzschentke and Schmidt, 2000) Taken

together, these studies demonstrate that the glutamate system in different regions of the 
$\mathrm{mPFC}$ is specifically involved in regulating the induction vs. expression of behavioral sensitization to psychostimulants.

Recent data has shown that the mPFC glutamate system is involved in the mechanisms whereby stress influences the reinstatement of drug seeking behavior. Restraint stress induced a much larger increase of extracellular glutamate in the prefrontal cortex than in the hippocampus and the basal ganglia (Moghaddam, 1993). Stressinduced $\mathrm{mPFC}$ dopamine release has been shown to be blocked by an AMPA/kainate receptor antagonist (6-cyano-7-nitroquinoxaline-2,3-dione) administered into the $\mathrm{mPFC}$ (Jedema and Moghaddam, 1994). Additionally, dopamine levels in the mPFC were reduced upon blockade of $\mathrm{mPFC}$ AMPA receptors in the absence of stress (Takahata and Moghaddam, 1998). Furthermore, inactivation of prelimbic mPFC by tetrodotoxin (TTX) blocked both foot shock and cocaine-induced reinstatement of cocaine seeking behavior (Capriles et al., 2003). However, infusion of the D1 receptor antagonist, SCH 22390 into prelimbic mPFC, only blocked stress-induced but not cocaine-induced reinstatement (Capriles et al., 2003). Taken together, these studies indicate that the glutamate system in the $\mathrm{mPFC}$ is involved in regulating $\mathrm{mPFC}$ dopamine transmission under basal and stressinduced conditions, and in doing so, the mPFC glutamate system might contribute to the stress-induced reinstatement of drug seeking (Takahata and Moghaddam, 1998; Capriles et al., 2003).

It has been reported that glutamate and dopamine levels increased in the NAcc core when extinguished cocaine SA behavior was reinstated by a cocaine-priming injection (McFarland et al., 2003). Interestingly, dopamine levels increased when saline control and cocaine-yoked rats were given a cocaine priming-injection (McFarland et al., 2003). Moreover, the enhanced NAcc glutamate release was independent of lever pressing. In the absence of a lever in the SA chamber during the reinstatement session, animals displayed a time course of enhanced of glutamate release similar to a group of animals with access to the lever (McFarland et al., 2003). Most importantly, inactivating dorsal $\mathrm{mPFC}$ by microinjecting the $\mathrm{GABA}_{\mathrm{A}}$ receptor agonist, muscimol, and the $\mathrm{GABA}_{\mathrm{B}}$ agonist, baclofen, blocked cocaine-priming induced reinstatement and glutamate release in the NAcc core, indicating that dorsal mPFC glutamatergic projections to the NAcc core play an important role in cocaine induced reinstatement of drug seeking behavior (McFarland et al., 2003). On the other hand, blockade AMPA/kainate or NMDA receptors in the NAcc core dose-dependently reduced cue-induced reinstatement of cocaine seeking behavior (Backstrom and Hyytia, 2007), suggesting that dorsal mPFC glutamatergic neurotransmission to NAcc core also might be involved in this form of reinstatement.

\subsubsection{The functional role of glutamate system in the VTA}

GluR1 homomeric AMPA receptors are $\mathrm{Ca}^{2+}$ permeable, and $\mathrm{Ca}^{2+}$ is important in initiating intracellular signaling cascades. Thus, drugs of abuse that upregulate GluR1 levels in the VTA might alter the membrane potential and intracellular signaling events that contribute to sensitization (Carlezon and Nestler, 2002). It has been reported that rats 
that showed behavioral sensitization after repeated cocaine treatment had the highest GluR1 levels in the VTA (Churchill et al., 1999). Consistent with the idea that upregulated GluR1 is associated with sensitization, chronic exposure to the D1 receptor antagonist, SCH 23390, which has been shown to block behavior sensitization to amphetamine (Vezina, 1996) and cocaine (Shippenberg and Heidbreder, 1995), reduced GluR1 levels in the VTA (Carlezon and Nestler, 2002). Taken together, GluR1 in the VTA might be involved in the cellular mechanisms underlying sensitization (Carlezon and Nestler, 2002).

The glutamate system in the VTA has been shown to be critical in the reinforcing effects of drugs of abuse. For example, blocking NMDA and AMPA receptors before a cocaine conditioning trial abolished the induction of cocaine conditioned place preference (CPP) (Harris and Aston-Jones, 2003). Heroin SA behavior was dose-dependently blocked by intra-VTA administration of AP5 or ketamine, both of which are NMDA receptor antagonists (Xi and Stein, 2002), indicating that NMDA receptors in VTA are involved in the reinforcing effects of heroin SA as well.

Ionotropic glutamate receptors in the VTA are also thought to play an important role in the reinstatement of drug seeking behavior. Bilateral perfusion of a nonselective iGluR antagonist, kynurenate, attenuated stress-induced cocaine seeking behavior (Wang et al., 2005). Moreover, blockade of ionotropic glutamate receptors by kynurenate dosedependently decreased cocaine-priming induced cocaine seeking behavior (Sun et al., 2005).

The VTA glutamate system undergoes neuroadaptation during acute and chronic treatment with drugs of abuse. Five drugs of abuse including amphetamine, cocaine, morphine, ethanol and nicotine have been shown to enhance the AMPAR to NMDAR EPSC ratio, suggesting an enhancement of AMPAR-mediated excitatory neurotransmission in the VTA (Saal et al., 2003). As long as 90 days post-withdrawal from cocaine SA, adaptation of ionotropic glutamate receptor expression has been reported in the VTA. NR1 was up-regulated as late as 90 days post-withdrawal, while GluR2 was up-regulated after only one day of withdrawal (Lu et al., 2003).

\subsubsection{The effects of the mPFC on the VTA}

In light of the above anatomical evidence for innervation between the $\mathrm{mPFC}$ and the VTA, especially the glutamatergic projection from $\mathrm{mPFC}$ to VTA, functional studies have revealed a critical role for these efferents in the regulation of VTA neuronal activity. One of the major functions of this projection is to regulate the activity of dopamine neurons in the VTA and the subsequent DA release of VTA afferent DA neurons, although neuroanatomical studies indicate that this would be an indirect, polysynaptic effect of mPFC glutamatergic projections - most likely to laterodorsal tegmental nucleus (Omelchenko and Sesack, 2007). 
The firing pattern of DA neurons in the VTA has been examined extensively, and, thus, complicated results have been reviewed and categorized into three groups (Tzschentke, 2001). A fundamental observation pertaining to our study was obtained from a midbrain slice preparation that demonstrated the firing rate of VTA dopamine neurons was increased by glutamate, NMDA, AMPA, and kainate without changing the burst firing activity (Seutin et al., 1990; Wang and French, 1993; Wang and French, 1993). Interestingly, when the VTA was perfused with NMDA through a microdialysis probe, the regulation of DA release in the NAcc appeared to be biphasic: low dose NMDA $(<100 \mu \mathrm{M})$ increased DA release in the NAcc, while high dose NMDA $(>100 \mu \mathrm{M})$ decreased DA release. Furthermore, the DA release in the NAcc was strongly correlated with the VTA DA neuron firing rate (Wang et al., 1994). This study suggested that glutamatergic stimulation in the VTA was involved in regulating DA release in the NAcc and DA neuron activity in the VTA. Direct bilateral stimulation of mPFC at intensities which have been shown to support intracranial self stimulation (ICSS) increased DA release by about $38 \%$ and $69 \%$ in the NAcc at $50 \mu \mathrm{A}$ and $100 \mu \mathrm{A}$ stimulus, respectively (Taber and Fibiger, 1995). Application of AP5 and CNQX in the VTA blocked the enhanced DA release in the NAcc during the cortical stimulation (Taber et al., 1995), suggesting that the glutamatergic projections from $\mathrm{mPFC}$ regulate the activity of DA neurons in the VTA possiblely through indirect, polysynaptic mPFC glutamatergic projections (Omelchenko and Sesack, 2007).

By using antero- and retrograde tracers, it is known that glutamatergic projections to the VTA are target-specific: PFC synaptic inputs selectively synapse on GABAcontaining mesoaccumbens neurons or DA-containing mesocortical neurons (Carr and Sesack, 2000). Interestingly, a neurochemical study using in vivo microdialysis supported this neuroanatomical observation. Intra-VTA reverse microdialysis of $100 \mu \mathrm{M} L Y 293558$, an AMPA/kainate antagonist, increased DA release in the NAcc by reducing the activity of GABA interneurons and, thereby, disinhibiting mesoaccumbens VTA DA neurons. In contrast, this compound reduced DA release in the PFC due to the inhibition of AMPA receptors on mesocortical VTA DA neurons (Takahata and Moghaddam, 2000). This observation suggests that mesocortical DA neurons are primarily under direct excitatory control from mPFC glutatamatergic inputs, while mesoaccumbens DA neurons are also affected indirectly by mPFC glutamatergic inputs to GABA interneurons (Takahata and Moghaddam, 2000). Further experiments would be necessary to determine whether tonic glutamatergic inputs differentially regulate VTA DA neurons or GABA interneurons in a target specific manner, e.g. whether tonic glutamatergic inputs to mesocortical DA neurons might be greater than to mesoaccumbens DA neurons or, alternatively, tonic glutamatergic inputs to GABA interneurons regulating mesoaccumbens VTA DA neurons might be greater than to those regulating mesocortical VTA DA neurons.

\subsection{Self-administration model in nicotine abuse}

Nicotine has been shown to be self-administered by several animal species, including primates (Goldberg, Spealman et al., 1981), dogs (Risner and Goldberg, 1983) and rodents (Corrigall and Coen, 1989; Valentine et al., 1997). The significance of these 
animal models of nicotine self-administration can be addressed in four ways: first, the fact that nicotine is self-administered by multiple species indicates that nicotine is a reinforcer (Corrigall, 1999); second, these are valid models for the pre-clinical testing of medication because of the obvious parallels between the human smoking behavior and animal self-administration (Corrigall, 1999); third, these models can be used to examine the motivational effects of nicotine, which are difficult to study in human subjects because of ethical concerns (Corrigall, 1999); fourth, these models can contribute to understanding the neuronal and neurochemical mediators of nicotine dependence (Corrigall, 1999).

The majority of studies of nicotine self-administration have utilized the intravenous route, since oral self-administration does not emulate the pharmacokinetics of smoking, which has a very rapid uptake of nicotine into the circulation and rapid delivery to the brain. In essence, intravenous nicotine self-administration is the best nicotine self-administration model available for studying the motivational circuits involved in the behavioral effects of nicotine. Besides recognizing the research value of this animal model, we acknowledge that there are also differences between animal models of nicotine SA and human smoking behavior. For example, the intravenous route versus inhalation of tobacco smoke (Rose and Corrigall, 1997) lacks the rich sensorymotor stimulation of the respiratory tract and the aroma of the smoke. These appear to make significant contributions to the reinforcing efficacy of nicotine.

Based on the schedule of access to nicotine, there are two models of intravenous nicotine self-administration: intermittent access and unlimited access models. Intermittent access has the advantage of relatively higher response rates per hour for nicotine because of the relatively short session time, which is useful in examining the effects of a short acting drug on nicotine SA within a limited time interval. It does not, however, emulate the unrestricted nature of human smoking behavior insofar as humans have relatively unlimited access to nicotine in cigarettes. The first unlimited access model was developed in 1979 by the Hanson group (Hanson et al., 1979). In their model, rats were pretreated with various doses of nicotine ranging from 0 to $0.06 \mathrm{mg} / \mathrm{kg} /$ infusion every 30 minutes over 48 hours (Hanson et al., 1979). Then, animals were allowed to self-administer nicotine at the same dose that they had previously received and with 24 hour unlimited access at an FR1 reinforcement schedule, in which each lever press results in the delivery of one nicotine infusion (Hanson et al., 1979). They found that nicotine-maintained responding was greater than saline control and dose dependent with the peak response at $0.03 \mathrm{mg} / \mathrm{kg} / \mathrm{dose}$, which is the dose we used in the dissertation work. However, the pretreatment also complicated the interpretation of the reinforcing effect of nicotine. In another unlimited access schedule study but without nicotine pretreatment, nicotine was self-administered at several doses, particularly at $0.03 \mathrm{mg} / \mathrm{kg}$ dose (Cox et al., 1984). However, the rate of responding on the inactive lever was high and dose-dependent. Thus, the specificity of this operant behavior was not established in these studies (Cox et al., 1984).

Our laboratory reported a revised procedure of unlimited access to nicotine selfadministration (Valentine et al., 1997), showing that rats individually housed in operant 
chambers were able to acquire nicotine self-administration at doses as low as $0.00375 \mathrm{mg} / \mathrm{kg}$ per infusion without previous training, shaping or food deprivation (Valentine et al., 1997). Moreover, the nicotine intake of rats ranged from 0.18 to 1.38 $\mathrm{mg} / \mathrm{kg}$, which is similar to the quantities self-administered by humans (Valentine et al., 1997). Furthermore, our laboratory examined the effects of rat strain differences on nicotine self-administration behavior. Inbred Lewis rats were more susceptible to nicotine SA than Sprague- Dawley or Fisher 344 rats (Brower et al., 2002); based on this study, male Lewis rats are the subjects of this dissertation research project. Thus, my dissertation work utilized addiction prone Lewis rats in our established model of unlimited access to nicotine self-administration in order to emulate nicotine selfadministration in humans who might be genetically susceptible to becoming dependent on the drug.

\subsection{Summary}

Despite the intensification of efforts to reduce smoking, nicotine abuse and addiction through cigarette smoking is still the leading preventable cause of death in the United States. Accumulating data implicates the mesocorticolimbic system in mediating the reinforcing effects of drugs of abuse. Multiple neurotransmitter systems within the mesocorticolimbic system including dopamine, glutamate, GABA and serotonin have been shown to be involved. The mesocorticolimbic system undergoes robust neuroadaptation during acute as well as chronic nicotine treatment and post-withdrawal. $\mathrm{mPFC}$ and VTA are two critical neuroanatomical regions within the mesocorticolimbic system. The mPFC provides the primary excitatory inputs to subcortical areas including the NAcc and the VTA, while VTA DA neurons project to NAcc and mPFC as well. Thus, the $\mathrm{mPFC}$ and VTA are functionally connected and reciprocally regulated. Several drugs of abuse induce neuroadaptive changes in the glutamate circuitry within the mPFC and the VTA. It has been hypothesized that the glutamate system in the VTA is important for the induction of cocaine sensitization while the glutamate system in the $\mathrm{mPFC}$ is important for the expression of cocaine sensitization. In contrast, it is unclear what roles the glutamatergic projections from mPFC to the VTA play during chronic nicotine SA and post-withdrawal. It has been shown that the VTA glutamate system is critical in mediating the reinforcing effects of drugs of abuse. Thus, the firing pattern of dopamine neurons in the VTA, which are critical to the acquisition of operant drug selfadministration, can be regulated by glutamatergic inputs. Utilization of a chronic nicotine self-administration model enables us to emulate human nicotine intake behavior in rats. The long-term changes in the mesocorticolimbic glutamate system that occur with chronic nicotine SA have not been reported. Thus, this dissertation focuses on neuroadaptive changes in the glutamate pathways within the mesocorticolimbic sytem during chronic nicotine SA and post-withdrawal. 


\subsection{Specific aims}

In light of the studies described above, the mesocorticolimbic pathway is critical in almost all aspects of drug abuse. Pyramidal neurons in the $\mathrm{MPFC}$ project glutamatergic fibers to the VTA and, in turn, receive dopaminergic inputs from the VTA. Though many of the neurochemical and molecular effects of nicotine have been well studied, the drug's long-term neuroadaptive effects, specifically within the mesocorticolimbic pathway, are largely undefined. A series of experiments have been designed to investigate: (1) the molecular neuroadaptive changes in ionotropic glutamate receptors during chronic nicotine self-administration and post-withdrawal; and (2) the neurochemical and behavioral changes that might result from these receptor changes. The working hypothesis of this dissertation is that there exists a hyperglutamatergic state of neurotransmission between the $\mathrm{mPFC}$ and VTA during chronic nicotine SA. The following are the Specific Aims of this project, which were examined in Chapters 2 and 3:

- To determine the changes in the expression of ionotropic glutamate receptors within the mesocorticolimbic pathway during chronic nicotine self-administration.

- To investigate the neurochemical as well as behavioral changes associated with these alterations in receptor expression during chronic nicotine SA and postwithdrawal. 


\section{Chapter 2. Selective Up-Regulation of Ionotropic Glutamate Receptor Subunits within Mesocorticolimbic Regions during Chronic Nicotine Self-administration*}

\subsection{Introduction}

The mesocorticolimbic system, which is involved in cognition, stress, and goaldirected motor activity, as well as addiction, involves the ventral tegmental area (VTA), nucleus accumbens (NAcc), prefrontal cortex (PFC), striatum (ST), hippocampus, and amygdala (Le Moal and Simon, 1991). Nicotine, the major psychoactive component of cigarette smoke, has been shown to stimulate mesocorticolimbic dopamine pathways, which originate in the VTA, resulting in dopamine (DA) secretion within the NAcc and PFC (Corrigall et al., 1992). In so doing, nicotine activates various subtypes of nicotinic cholinergic receptors (nAChRs) located on dopaminergic neurons, GABAergic neurons, and glutamatergic terminals within the VTA, and on dopamine terminals in the projection regions (e.g. NAcc) (Corrigall et al., 1992). This nicotinic stimulation of dopamine secretion is essential for the reinforcing effects of nicotine (Corrigall et al., 1992). However, other neurotransmitter pathways, such as glutamatergic neurons projecting from PFC to VTA, also are involved in the motivational effects of nicotine (Watkins et al., 2000; Picciotto and Corrigall, 2002).

Synaptic glutamate binds to N-methyl-D-aspartate (NMDA) and $\alpha$-amino-3hydroxy-5-methyl-4-isoxazole propionic acid (AMPA) receptors, opening postsynaptic cation channels that mediate fast excitatory neurotransmission (Nakanishi et al., 1998). Recent reports have shown that nicotine stimulated VTA glutamate release through presynaptic nicotinic acetylcholine receptors, which in turn excited VTA dopaminergic neurons (Fu et al., 2000; Mansvelder and McGehee, 2000). Nicotine induced glutamate release through $\alpha 7$ subunit-containing nAChRs on glutamate terminals in a VTA slice preparation (Mansvelder and McGehee, 2000). Ultrastructural and in vivo microdialysis studies support the idea that the excitation of VTA dopamine neurons is primarily dependent on glutamatergic efferents from PFC (Sesack and Pickel, 1992; Taber et al., 1995). Using in vivo microdialysis, our lab also showed that nicotine dose-dependently stimulated DA release in NAcc, an effect that was abolished by infusing NMDA antagonists (AP-5 or CGS 19755) into VTA (Fu et al., 2000). These findings demonstrate the pivotal role played by glutamatergic pathways in the excitation of mesocorticolimbic dopaminergic neurons by nicotine.

* Chapter 2 reprinted by permission of the publisher: Wang, F., Chen, H., Steketee, J.D. and Sharp, B.M. (2007) Upregulation of ionotropic glutamate receptor subunits within specific mesocorticolimbic regions during chronic nicotine self-administration.

Neuropsychopharmacology, 32, 103-9. 
Nicotine, like other drugs of abuse, elevated the ratio of AMPA/NMDA excitatory post-synaptic currents (EPSC) in midbrain dopamine neurons, consistent with enhanced dopaminergic neurotransmission (Saal et al., 2003). Nicotine also enhanced NMDA and/or AMPA-induced synaptosomal release of norepinephrine from the hippocampus and dopamine from the striatum (Risso et al., 2004). These functional studies suggest that nicotine may up-regulate ionotropic glutamate receptors but do not provide insight into the effects of nicotine on the actual expression of ionotropic glutamate receptors in specific brain regions. The expression of GluR1 mRNA in VTA has been found to be increased after acute injection of nicotine directly into VTA (Ferrari et al., 2002). However, the effects of chronic nicotine self-administration on ionotropic glutamate receptor protein levels have not, to our knowledge, been studied. The aim of this study was to determine whether chronic nicotine self-administration modulates protein levels of ionotropic glutamate receptor subtypes within mesocorticolimbic regions. To accomplish this, Western blotting with subunit specific antibodies was used to obtain semi-quantitative comparisons within the VTA, PFC, NAcc and ST.

\subsection{Materials and methods}

\subsubsection{Materials}

(-)-Nicotine hydrogen tartrate (all doses calculated as free base) was purchased from Sigma (St. Louis, MO). Operant chambers, circuit boards, interface modules and SA software were from Coulbourn Instruments (Allentown, PA).

\subsubsection{Nicotine self-administration}

Self-administration was performed according to our previously published protocol with minor modifications (Valentine et al., 1997). Seven days after acclimation to a reverse light cycle and handling, male Lewis rats (Harlan Sprague Dawley, Indianapolis, IN), weighing 250-350g, received jugular cannulae under xylazine-ketamine anesthesia (13 and $87 \mathrm{mg} / \mathrm{kg}$, i.m., b.wt., respectively; Parke-Davis, Morris Plains, NJ) and then were immediately placed into individual operant chambers where they resided for the duration of the study. Rats were allowed to recover for $3 \mathrm{~d}$, during which time they received hourly injections of heparin $(100-200 \mathrm{U} / \mathrm{ml}$ in $50 \mu \mathrm{l})$ and a daily injection of the antibiotic Baytril (7.6 mg/kg in $0.1 \mathrm{ml}$, i.v.; Bayer Corp., Shawnee Mission, KS). Then rats were randomly assigned to treatment groups and jugular lines were filled with either $0.03 \mathrm{mg} / \mathrm{kg}$ nicotine in $200 \mathrm{U} / \mathrm{ml}$ heparinized saline $(50 \mu \mathrm{l}$ delivered over $0.81 \mathrm{sec}$ per 300 gm body weight) or heparinized saline alone. The nicotine solution was freshly prepared every 7-14 day and stored in a foil-wrapped glass bottle at $4^{\circ} \mathrm{C}$. Every day, the nicotine solution remaining in the syringe was discarded and the syringe was refilled.

Each sound-attenuating environmental enclosure was equipped with its own ventilation fan and house light; the light was scheduled to turn off daily at exactly 10:30 
A.M. and on again at 10:30 P.M. The interior operant chamber contained two levers positioned $5 \mathrm{~cm}$ above the floor and a green cue light $1 \mathrm{~cm}$ above each that signaled the availability of nicotine. One lever was randomly assigned as the active bar and would signal the computer-driven pump to deliver a $50 \mu 1 / 0.81 \mathrm{sec}$ bolus injection of nicotine or saline when pressed by the rat; pressing the other bar had no consequence. Injections were followed by a $7 \mathrm{sec}$ time-out, during which the green light above the active bar was not illuminated and nicotine or saline were unavailable. Rats were not shaped, conditioned, or food deprived in preparation for lever pressing and were allowed to acquire selfadministration behavior throughout the $23 \mathrm{hr}$.

Environmental enclosures, operant chambers, and syringe pumps were controlled by Coulbourn Habitest Universal LabLink interfaces and computers located in an adjacent room. Each morning, during the final hour of the lights-on cycle (i.e., at 9:30 A.M.), the clock for the computer program (Coulbourn L2T2) had to be manually reset, necessitating a short interruption in nicotine availability. To accommodate this, as well as to accomplish animal husbandry needs, measurements of body weight, and data downloading, all of the environmental enclosures were opened and the green cue lights were turned off to signal this interruption to the rats. Levers were not retracted, and lever press activity was not recorded. Exactly $1 \mathrm{~h}$ later (10:30 A.M.), house lights were switched off, cue lights were illuminated, nicotine (or saline) was again available, and enclosure doors were shut. All procedures were conducted in accordance with the $N I H$ Guidelines Concerning the Care and Use of Laboratory Animals and were approved by the Animal Care and Use Committee of the University of Tennessee.

Rats were housed in self-administration chamber for 18 days. By day 8, stable nicotine self-administration had been achieved in that active lever presses were two-fold greater than inactive in each animal. All animals continued to self-administer nicotine until day 18.

\subsubsection{Brain punches and tissue preparation}

On day 19, after rats had access to nicotine for $4 \mathrm{~h}$, they were anesthetized with isoflurane and decapitated. The average time interval between the last active lever press for nicotine and removal of the animals from operant chambers was $31.8 \mathrm{~min}$; thus, rats were untethered from their chambers $3 \mathrm{~h}$ and $28.2 \pm 18.6$ min (mean \pm SD) after the start of the session. Rat brains were removed and stored at $-80^{\circ} \mathrm{C}$. Frozen brain sections of 0.7 $\mathrm{mm}$ thickness were obtained using arrays of 20 double-edge razor blades (Professional Medical Products Inc. Greenwood, SC), designed and constructed in our laboratory. The blade arrays were assembled using two screws with matching wing-nuts. One $0.7 \mathrm{~mm}$ stainless steel washer per screw (Small Parts, Inc. Miami Lakes, FL) was used to separate each blade. Brains were first equilibrated at $-20^{\circ} \mathrm{C}$ for $10 \mathrm{~min}$. The ventral surface of the brain was then placed on top of the blade array and was gently and briefly pressed downward. Care was taken to ensure the rostral-caudal axis of the brain was perpendicular to the blades (for coronal sections). The brain and the blades then were turned upside down and set on top of a cold $\left(-10^{\circ} \mathrm{C}\right)$ metal plate (FTS systems, Stone 
Ridge, NY). Using a slight rocking motion, a metal block was applied to gradually press the entire brain into the blades, insuring that the brain was completely embedded within the blades. The assembly then was disassembled on powdered dry ice, and individual brain slices were laid flat on a cold $\left(-10^{\circ} \mathrm{C}\right)$ surface. Brain areas of interest were identified with a dissecting microscope, according to the atlas of Paxinos and Watson (1986), and punched out using a 20 -gauge syringe adapter. Excised tissue was immediately transferred to tubes embedded in dry ice and stored at $-80{ }^{\circ} \mathrm{C}$ until further processing. Dissected tissues were sonicated in 2\% sodium dodecyl sulfate (SDS) solution for $30 \mathrm{sec}$ in an ice water bath. Sonicated tissues were centrifuged at $800 \times \mathrm{g}, 5 \mathrm{~min}$. Protein concentrations were measured using the bicinchoninic acid assay (Pierce, Rockford, IL) with bovine serum albumin (BSA) as the standard.

\subsubsection{Western blotting}

To determine ionotropic glutamate receptor expression level differences after treatment, samples of the same brain region from both treatment groups (nicotine vs. saline) were loaded in alternating sequence on the same gel.

Samples were diluted in Laemmli buffer (Bio-Rad, Hercules, CA) at a ratio of $1: 2$, and were boiled for $5 \mathrm{~min}$., loaded, and then separated on $10 \%$ polyacrylamide gels in a buffer containing $0.1 \%$ SDS, $192 \mathrm{mM}$ glycine, and $25 \mathrm{mM}$ Tris, $\mathrm{pH}$ 8.3. Proteins were then transferred to polyvinylidene difluoride membranes (Bio-Rad, Hercules, CA) in a buffer containing $192 \mathrm{mM}$ glycine, $20 \%$ methanol and $25 \mathrm{mM}$ Tris, $\mathrm{pH} 8.3$ at $4^{\circ} \mathrm{C}$ overnight. After transfer, membranes were washed in Tris- buffered saline with Tween20 (TBST; $100 \mathrm{mM}$ Tris and $0.9 \%$ sodium chloride with $0.1 \%$ Tween 20). Membranes were blocked with agitation by $5 \%$ non-fat dried milk for 2 hours at room temperature (RT). Based on the molecular weight differences between NMDA subunits, AMPA subunits, and $\beta$-actin, blots were carefully cut into three separate sections by visualizing the protein molecular weight markers: upper blots were used to detect NMDA receptor subunits, middle blots to detect AMPA receptor subunits, and lower blots to detect $\beta$ actin in order to evaluate protein loading. These blots were incubated with selective antibodies against $\beta$-actin (1:1000; Sigma, St. Louis, MO), glutamate receptor subunit 1 (GluR1; 1:1000;Upstate, Lake Placid, NY), GluR2/3 (1:1000; Upstate, Lake Placid, NY), NMDA receptor subunit 1 (NR1; 1:400; Santa Cruz, CA), NR2A (1:200; Santa Cruz, CA), and NR2B (1:500; Upstate, Lake Placid, NY). Blots were then washed in TBST and incubated in horseradish peroxidase-conjugated secondary antibodies (1:3000 for $\beta$-actin, 1:1500 for detecting the others). All antibodies were diluted in the same blocking solution and incubated for $2 \mathrm{hr} \mathrm{RT}$ or overnight at $4^{\circ} \mathrm{C}$. Target proteins were visualized by Supersignal (Pierce, Rockford, IL) followed by imaging using a Bio-Rad ChemiDoc instrument with quantitation by Quantity One software (Bio-Rad, Hercules, CA). 


\subsubsection{Data analysis}

To determine the specificity of nicotine self-administration, the number of active versus inactive lever presses per day was compared within each treatment group (i.e., nicotine and saline). Data are presented as mean \pm S.E.M. Comparisons of active versus inactive lever presses within treatment groups were made by two-way analysis of variance, using SPSS statistical software, version 12.0 (SPSS Inc., Chicago, IL).

Each sample from a rat was run once on each of two gels. Optical density values for each lane were normalized to $\beta$-actin to control for variation in loading and transfer. For each sample, normalized values that had been averaged between the two blots were used to conduct statistical comparisons (independent samples t-test) to identify treatment effects within a region for each subunit. The mean values of nicotine treatment groups were expressed as a percentage of saline \pm S.E.M. Treatment differences were considered significant at $\mathrm{p}<0.05$.

\subsection{Results}

\subsubsection{Nicotine self-administration}

The numbers of daily active vs. inactive lever presses (mean \pm S.E.M.) are presented in Figure 2.1. In the nicotine treatment group, the mean level of active lever presses by rats receiving a programmed injection of i.v. nicotine immediately after each active lever press was approximately twice the inactive presses. In this group, active lever presses were significantly higher than inactive $\left(\mathrm{F}_{1,10}=51.49, p<0.001\right)$, whereas no difference was observed in the saline group $\left(\mathrm{F}_{1,10}=3.68, p>0.05\right)$. A comparison of active lever presses in the nicotine $v s$ saline groups also showed a significant treatment effect $(\mathrm{F}=9.57, p=0.002)$.

\subsubsection{Chronic nicotine self-administration-induced up-regulation of NMDA and AMPA receptor subtypes within mesocorticolimbic regions}

In all immunoblots, only one band was visualized. Representative gels from each group are shown in Figures 2.2-2.5. NMDA receptor NR2A and NR2B subunits were visualized at a molecular weight of $180 \mathrm{kDa}$. Chronic nicotine SA significantly increased NR2A and NR2B subunit expression levels in PFC by $67 \%(p=0.04)$ and $83 \%(p=$ 0.027 ), respectively (Figure 2.2 ). The GluR2/3 subunit was visualized at $110 \mathrm{kDa}$. In VTA, chronic nicotine SA preferentially up-regulated the GluR2/3 receptor expression level by $34 \%$ ( $p=0.011$; Figure 2.3). In contrast, Figures 2.4 and 2.5 show that NAcc and striatal NMDA and AMPA receptor subunit levels were unaffected by chronic nicotine SA. 


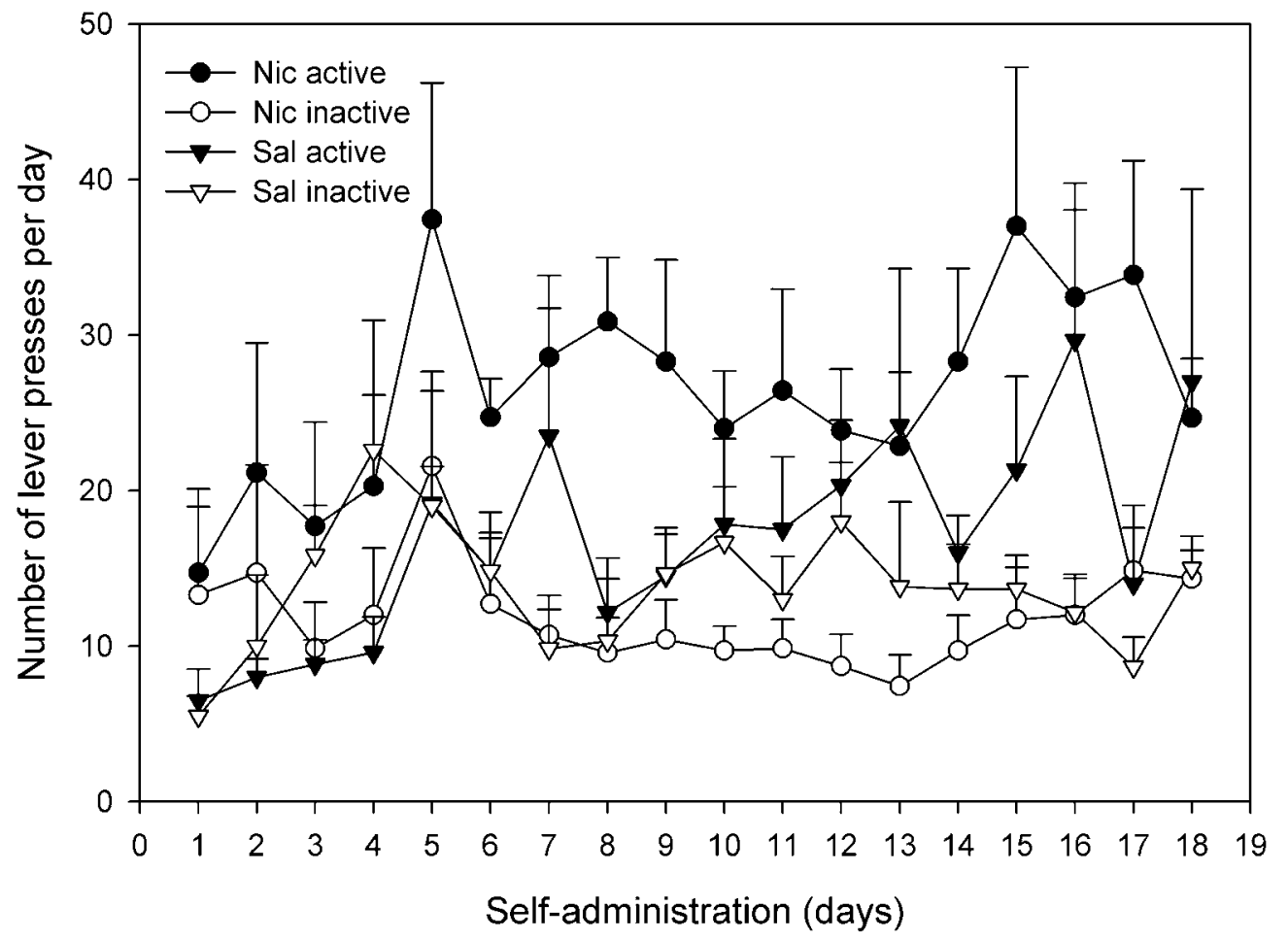

Figure 2.1. Lever-pressing activity during 18 days of nicotine (Nic) selfadministration compared to saline (Sal). The mean numbers ( \pm S.E.M.) of lever presses (active or inactive) per day for the four data sets were analyzed by two-way analysis of variance. In the nicotine group, the difference between active vs. inactive lever presses was significant $(\mathrm{F}=38.79, p=0.002 ; \mathrm{n}=7)$, but not in the saline group $(\mathrm{F}=$ $0.53, p>0.05 ; \mathrm{n}=6$ ). Brain tissues from these animals were used in the immunoblots shown in Figures 2.2-2.5. 


\section{PFC}

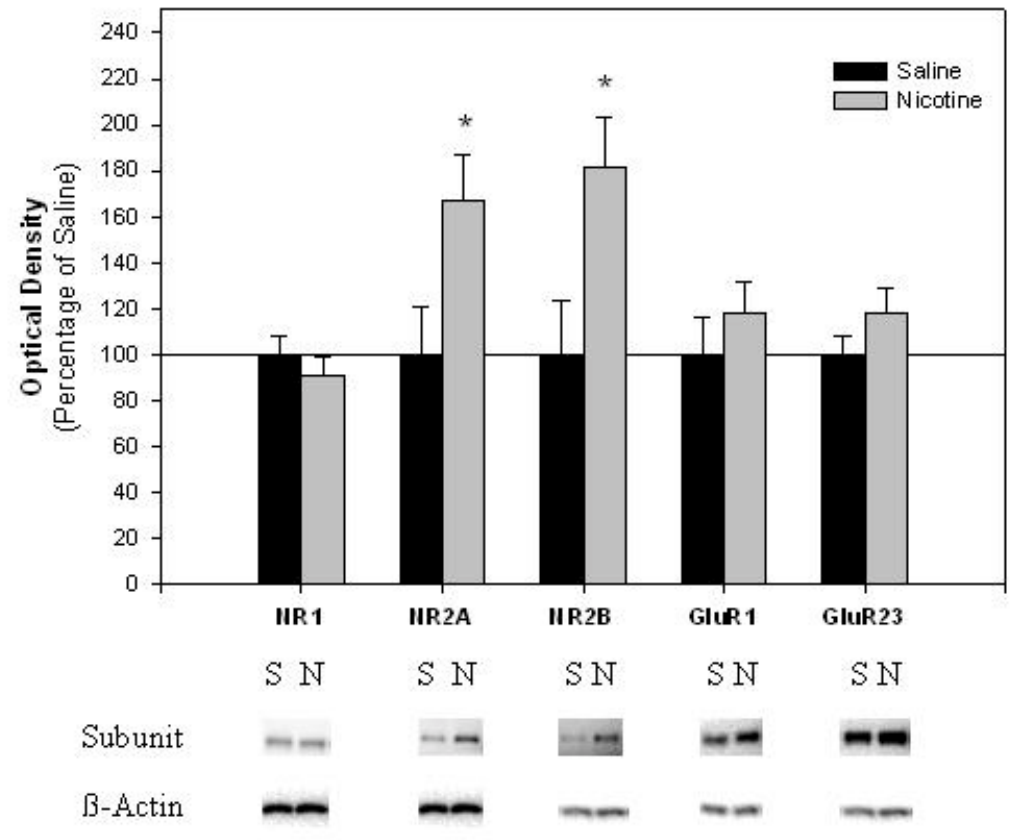

Figure 2.2. Nicotine self-administration (18 days) up-regulated NR2A and NR2B subunit levels within the prefrontal cortex (PFC) of rat brain. Nicotine induced significant increases (67\% and 83\%) in NR2A and NR2B subunit immunoreactivities within the PFC. Data were normalized to $\beta$-actin levels and converted to mean percent of saline \pm S.E.M. for each subunit (nicotine $n=7$, saline $n=6$ ). Representative blots comparing saline $(\mathrm{S})$ vs nicotine $(\mathrm{N})$ treated animals are shown for each subunit. *, significantly different from saline; $p<0.05$, unpaired t-test. 


\section{VTA}

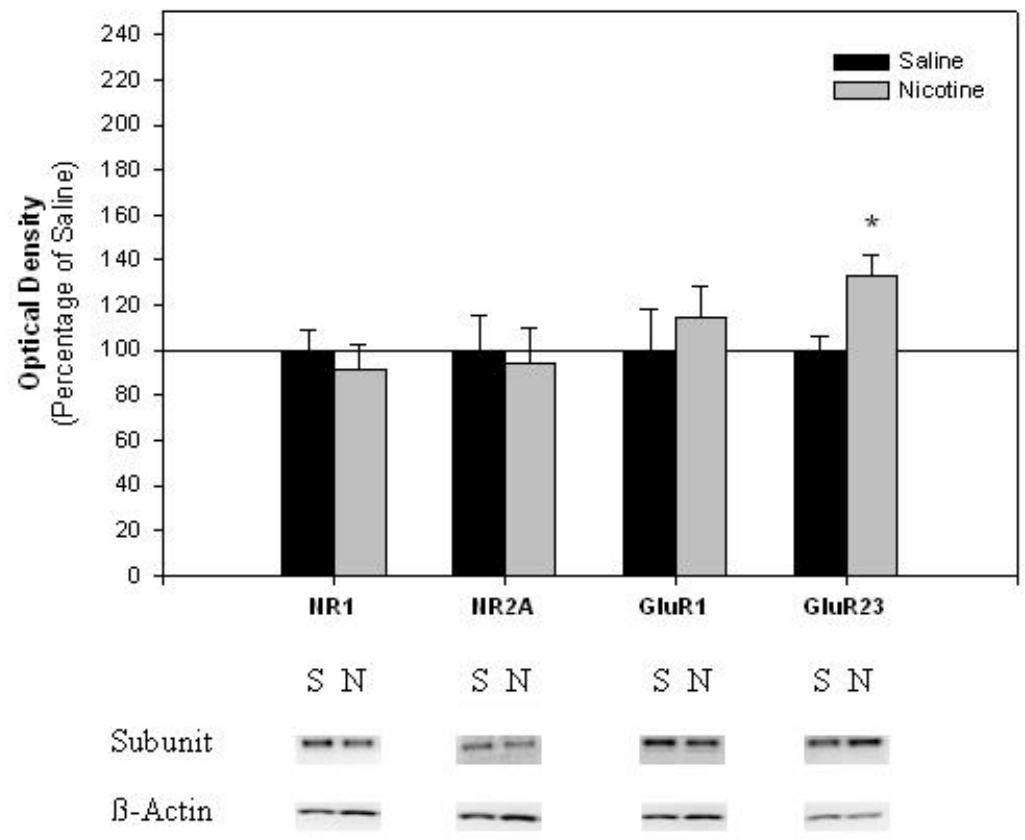

Figure 2.3. Nicotine self-administration (18 days) increased GluR2/3 subunit immunoreactivity within the ventral tegmental area (VTA) of rat brain. Nicotine increased GluR2/3 subunit levels in this region by $34 \%$ (nicotine $n=7$, saline $n=6$ ). Representative blots comparing saline $(\mathrm{S})$ vs nicotine $(\mathrm{N})$ treated animals are shown for each subunit. $*$, significantly different from saline; $p<0.05$, unpaired t-test. 


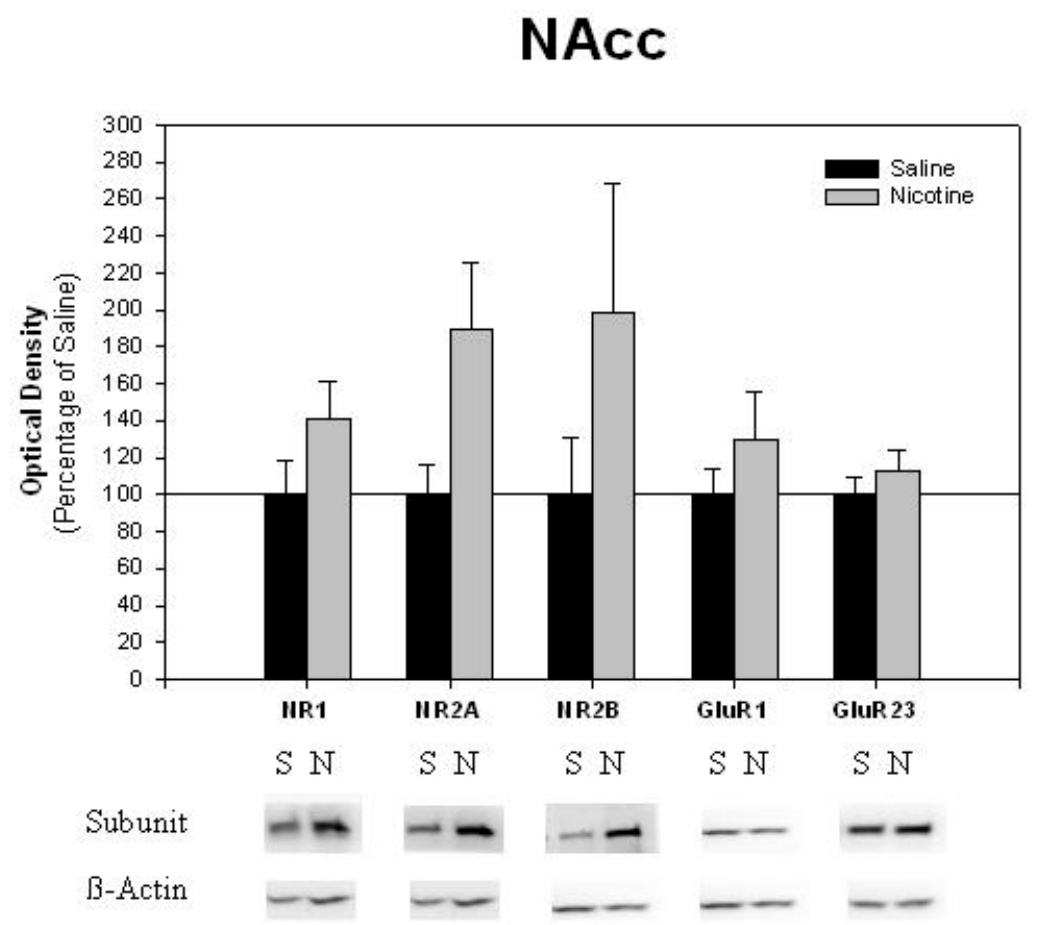

Figure 2.4. Nicotine self-administration (18 days) produced no statistically significant alterations in any of the subunits within the nucleus accumbens (NAcc) shell of rat brain. Representative blots comparing saline $(\mathrm{S})$ vs nicotine $(\mathrm{N})$ treated animals are shown for each subunit (nicotine $n=7$, saline $n=6$ ). 


\section{ST}

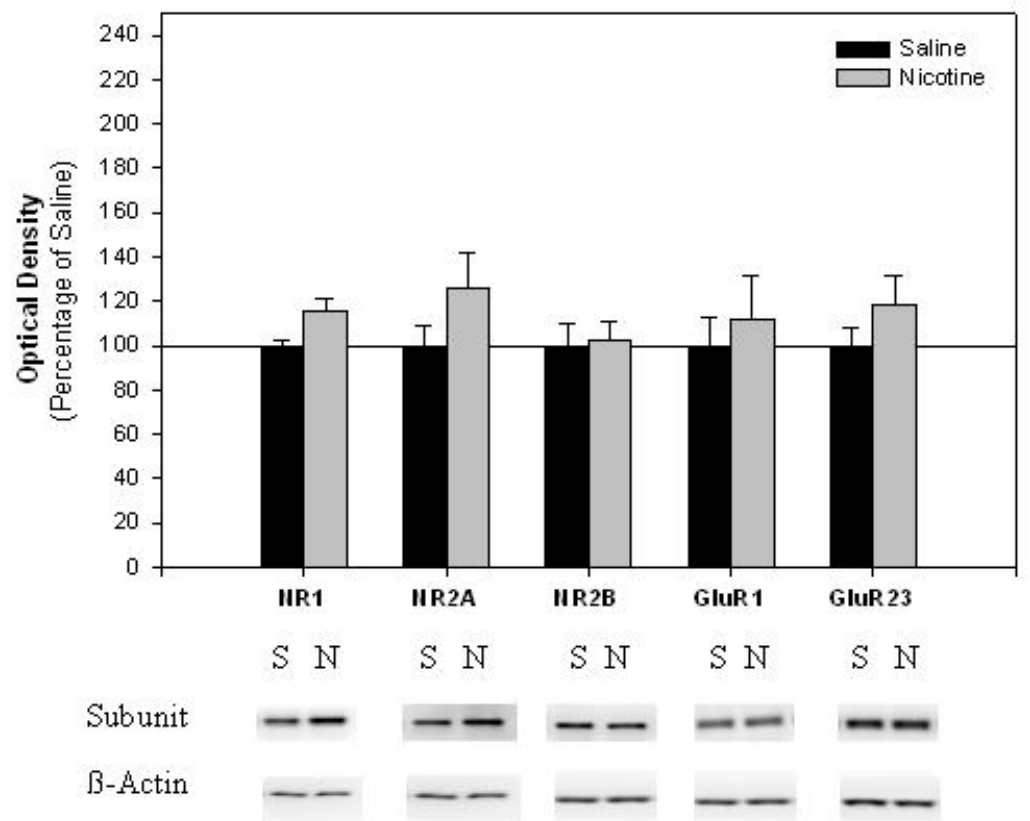

Figure 2.5. Nicotine self-administration (18 days) produced no statistically significant alterations in any of the subunits within the striatum (ST) of rat brain. Representative blots comparing saline $(\mathrm{S})$ vs nicotine $(\mathrm{N})$ treated animals are shown for each subunit (nicotine $n=7$, saline $n=6$ ). 
The relatively large variance in NR2A levels in NAcc (Figure 2.4) and the statistical tendency toward a difference between treatment groups $(p=0.06)$ prompted a second experiment. NR2A was unaffected by nicotine SA (immuno- blot optical density, expressed as a percentage of the saline group: $100 \pm 12.6$ vs $109.6 \pm 27.5 \%$ (values are mean \pm SEM) for saline vs nicotine, respectively; $\mathrm{p}>0.05, \mathrm{n}=6$ per treatment group). For comparison, NR1 determinations were also repeated. As previously reported, no

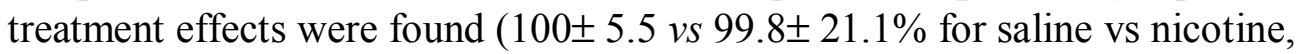
respectively; $\mathrm{p}>0.05, \mathrm{n}=6$ per treatment group).

\subsection{Discussion}

In rats chronically self-administering nicotine, several glutamate receptor subunits were up-regulated within the mesocorticolimbic pathway. These include NR2A and NR2B receptor subunits within PFC and GluR2/3 subunits within VTA. These findings demonstrate that chronic nicotine SA selectively modulates ionotropic glutamate receptor subunit expression levels, suggesting that glutamatergic neurotransmission is altered within the mesocorticolimbic pathway.

One study determined the response of ionotropic glutamate receptor mRNA levels, but not protein subunits, to chronic treatment with nicotine in vivo. It indicated that chronic nicotine exposure during postnatal days 8- 12 significantly increased NR2A mRNA levels in auditory cortex, whereas thalamic NR2B mRNA levels declined (Hsieh et al., 2002). This finding suggests that chronic exposure to nicotine affects the transcription of specific ionotropic glutamate receptor subunits in certain brain regions. However, subunit expression was only studied during the period of rat postnatal brain maturation equivalent to the third trimester of human brain development.

Several drugs of abuse have been shown to impact the function of ionotropic glutamate receptors. A recent study suggested that glutamatergic neurotransmission was altered after a single exposure to several drugs of abuse (Saal et al., 2003). In that study, 24- $30 \mathrm{~h}$ after in vivo exposure (i.p. injection) to nicotine, morphine, ethanol, cocaine or amphetamine, the strength of excitatory synapses on dopamine neurons was enhanced, reflecting an increase of AMPA/NMDA EPSC (Saal et al., 2003). However, the molecular mechanism(s) underlying these synaptic effects on midbrain dopamine neurons was not identified. The time interval between drug exposure and the detection of increased synaptic strength was probably sufficient for increased ionotropic glutamate subunit expression to have occurred.

One may postulate that chronic exposure to abused drugs can induce an increase in AMPA receptor subunit expression. This is supported by experiments showing that chronic cocaine treatment up-regulated GluR1 and NR1 subunit levels in VTA, and that escalating doses of morphine also enhanced the expression of GluR1 subunits in VTA (Fitzgerald et al., 1996). In addition, after 3 days of withdrawal from repeated administration of cocaine or amphetamine, the direct application of AMPA onto VTA dopamine neurons resulted in increased glutamate responsiveness of these neurons 
(Zhang et al., 1997). Consistent with these findings, in the current study, chronic nicotine SA increased GluR $2 / 3$ subunit levels by $30 \%$ in VTA. This finding suggests that there might be a larger pool of recycling GluR2/3 subunits in VTA neurons since these subunits are known to recycle constitutively, which is important for the maintenance of basal synaptic activity (Passafaro et al., 2001; Shi et al., 2001). We also speculate that the AMPA/NMDA EPSC ratio may be increased if more GluR2/3 subunits reside in the plasma membrane. Interestingly, immunocytochemical studies have shown that GluR2/3 subunits were found in $63 \%$ of tyrosine hydroxylase $(\mathrm{TH})$ - positive VTA neurons, whereas GluR1 subunits were in $20 \%$ (Chen et al., 2001). Thus, the up-regulated GluR2/3 subunits induced by chronic nicotine SA might be present in 'silent synapses', which had no AMPA receptors prior to nicotine SA, and in the GluR1/TH doublepositive neurons, as well as GABAergic interneurons within VTA.

All drugs of abuse appear to enhance dopamine secretion in the NAcc (Berridge and Robinson, 1998). Nicotine does so in part through presynaptic $\alpha 7$ subunit-containing nicotinic receptors, located on glutamatergic terminals, which synapse on dopaminergic neurons within VTA (Mansvelder and McGehee, 2000). Nicotine stimulates glutamate release, increasing dopaminergic neurotransmission (Mansvelder and McGehee, 2000). Since the primary excitatory inputs to VTA originate in the PFC (Picciotto and Corrigall, 2002), enhanced excitatory input based on PFC- dependent mechanisms also may be a factor. We found that PFC NR2A and NR2B subunit levels were up-regulated by approximately $67 \%$ and $83 \%$, respectively, after nicotine SA. As the dendrites and soma of PFC glutamatergic neurons, which project to VTA, themselves receive glutamatergic inputs (Steketee, 2003), it is conceivable that the increased expression of PFC NMDA receptors containing NR2A and NR2B enhance the excitatory glutamatergic input to VTA. Similarly, a previous study found that chronic ethanol exposure increased NMDA (i.e., NR1, NR2A and NR2B) and AMPA (i.e., GluR2/3) receptor subunit levels in primary cortical cultures, suggesting that increased activity of PFC glutamatergic neurons may be a mechanism common to multiple drugs of abuse (Chandler et al., 1999).

Previous studies indicate that availability of NR2 subunits may be a limiting variable in the expression of intact NMDA receptors on the plasma membrane, as about $50 \%$ of NR1 is located in cytoplasmic pools while approximately $90 \%$ of NR2B is located at the cell surface (Hall and Soderling, 1997). Although the NR2B subunit appears to be constitutively trafficked to the synapse, NR2A overexpresson leads to its synaptic incorporation in an activity- dependent manner (Barria and Malinow, 2002). Collectively, theses observations suggest that the nicotine SA- induced increase in PFC NR2A and 2B are likely to be incorporated in functional NMDA receptors.

Compared with VTA, NAcc may be less sensitive to the excitatory effects of glutamate, as evident in single cell recordings in vivo, made on the fifth day of treatment with cocaine or amphetamine (White et al., 1995). The overall current response of VTA dopamine neurons to glutamate was significantly elevated in amphetamine and cocaine treated rats, whereas this was not observed in NAcc neurons (White et al., 1995). Consistent with this, we did not observe significant changes in the expression of either NMDA or AMPA receptor subunits in the dopamine terminal regions (e.g., NAcc and 
ST) of animals chronically self-administering nicotine; NR2A showed only a tendency to increase in NAcc. This indicates the regional specificity of the changes in glutamate receptor subunit expression induced by chronic nicotine SA. Although these regional changes in glutamate receptor subunit expression may impact neuronal function, perhaps underlying alterations in the glutamatergic responsiveness of VTA dopamine neurons exposed to drugs of abuse, additional studies beyond Western immunoblotting are necessary to elucidate the implications of the present findings. Furthermore, other adaptive changes (e.g., post-translational changes such as phosphorylation) may occur in some regions where protein levels were unaffected (White et al., 1995).

To our knowledge, this is the first report of altered ionotropic glutamate receptor subunit expression during chronic nicotine SA. Based on these findings, we hypothesize that there exists a hyperglutamatergic state within the mesocorticolimbic pathway during chronic nicotine SA (Kalivas, 2004). This may be involved in aspects of drug SA, such as sensitization, that reflect the occurrence of increasing neuronal plasticity with duration of exposure (Wolf et al., 2004). Further investigation will be needed to evaluate the neurochemical changes underlying this hypothesis. In summary, chronic nicotine SA selectively up-regulated NR2A and NR2B receptor subunits in PFC and GluR2/3 subunit in VTA. These findings may implicate hyperglutamatergic neurotransmission within the mesocorticolimbic dopaminergic circuitry. 


\section{Chapter 3. Neuroadaptive Changes in the Mesocortical Glutamatergic System during Chronic Nicotine Self- Administration and after Extinction in Rats}

\subsection{Introduction}

Drug abuse is associated with molecular and cellular neuroadaptation within the mesocorticolimbic system (Koob, 1992; Nestler, 1992; Hyman et al., 2006). This system includes the medial prefrontal cortx ( $\mathrm{mPFC})$, which provides the primary glutamatergic excitatory input to subcortical areas such as the nucleus accumbens (NAcc) and ventral tegmental area (VTA), and the VTA whose dopaminergic efferents regulate NAcc medium spiny neurons and mPFC pyramidal neurons (Sesack and Pickel, 1992; Taber et al., 1995; Omelchenko and Sesack, 2007). mPFC is instrumental to many dimensions of drug abuse including the expression of behavioral sensitization to psychostimulants (Wolf et al., 1995; Li and Wolf, 1997; Li et al., 1999; Li et al., 1999) and drug-primed reinstatement of drug-seeking behavior (McFarland et al., 2003). Within the MPFC and VTA, glutamatergic neurotransmission adapts to nicotine, reflecting changes in the expression and function of nicotinic acetylcholine receptors (nAChRs) and ionotropic glutamate receptors (iGluRs) (Mansvelder and McGehee, 2000; Mansvelder and McGehee, 2002; Saal et al., 2003; Wang et al., 2007).

Nicotine activates VTA nAChRs that stimulate dopamine release in the NAcc, which is essential for nicotine self-administration (SA) (Corrigall et al., 1992). Glutamatergic efferents from $\mathrm{mPFC}$ have an important role in the regulation of NAcc dopamine (DA) levels (Sesack and Pickel, 1992; Taber et al., 1995). For example, blockade of ionotropic glutamate receptors in the VTA, decreased basal NAcc dopamine by $30 \%$ and blocked DA release evoked by $\mathrm{mPFC}$ stimulation (Taber et al., 1995). Recent studies indicate that these neurochemical changes are mediated by a polysynaptic glutamatergic pathway from mPFC that probably innervates the lateral dorsal tegmental nucleus (LDTg) and pedunculopontine tegmental nucleus (PPTg), which in turn projects glutamatergic fibers to VTA mesoaccumbens DA neurons (Taber et al., 1995; Carr and Sesack, 2000; Omelchenko and Sesack, 2005). In addition, mPFC pyramidal neurons selectively innervate VTA mesocortical DA neurons (Carr and Sesack, 2000), which in turn affect pyramidal neuron activity (Harte and O'Connor, 2004). Thus, the activation of both polysynaptic and monosynaptic glutamatergic pathways, which originate in $\mathrm{mPFC}$, appears to regulate VTA glutamate levels. The polysynaptic pathway that includes the PPTg appears to be involved in nicotine SA (Corrigall et al., 2001).

To evaluate the significance of enhanced mPFC and VTA ionotropic glutamate receptor subunit expression during and after chronic nicotine SA (Wang et al., 2007), we first determined the effects of mPFC NMDA on glutamate neurotransmission by using dual probe microdialysis to measure extracellular glutamate levels in both $\mathrm{mPFC}$ and VTA. The expression of ionotropic glutamate receptor subunits was also determined after 10 days of extinction from chronic nicotine SA. In addition, we investigated the potential 
roles of the up-regulated $\mathrm{mPFC}$ and VTA ionotropic glutamate receptors in the maintenance of nicotine SA by microinjecting the NMDA or AMPA receptor antagonists, 2-amino-5-phosphonopentanoic acid (AP-5) or 1,2,3,4-tetrahydro-6-nitro-2,3-dioxobenzo[f]quinoxaline-7-sulfonamide (NBQX), into these respective regions and measuring lever press behavior in rats that chronically self-administered nicotine compared to sucrose pellets.

\subsection{Materials and methods}

\subsubsection{Materials}

(-)-Nicotine hydrogen tartrate (doses expressed as free base), AP-5 and NBQX were purchased from Sigma-Aldrich (St. Louis, MO). Sodium dihydrogen phosphate monohydrate, EDTA, methanol, and phosphoric acid were obtained from Fisher Scientific Co. (Fair Lawn, NJ). Cellulose fiber tubing was purchased from Spectrum (Laguna Hills, CA), and silica tubing (outer diameter, $148 \mu \mathrm{m}$; inner diameter, $73 \mu \mathrm{m}$; TSP 075150) was obtained from Polymicron Technologies Inc. (Phoenix, AZ). Operant chambers, circuit boards, interface modules and SA software for chronic nicotine SA and sucrose SA were purchased from Coulbourn Instruments (Allentown, PA) and Med Associates Inc. (Georgia, VT), respectively.

\subsubsection{Animals and surgeries}

Adult male Lewis rats (250-270 g, Harlan, Madison, WI) were given access ad libitum to standard rat chow and water. Rats were individually housed on a 12-h reversed light cycle (off at 10 A.M., on at 10 P.M.). After $7 \mathrm{~d}$ of housing under these conditions, rats were anesthetized with xylazine-ketamine (13 and $87 \mathrm{mg} / \mathrm{kg}$ b.wt., i.m.; SigmaAldrich), and chronic guide cannulae (20-gauge) were stereotaxically implanted into the ipsilateral mPFC and VTA for the in vivo microdialysis experiment according to the coordinates of Paxinos and Watson (Paxinos and Watson, 1986). For microinjection experiments, chronic microinjection cannulae (26-gauge) were stereotaxically implanted into the bilateral mPFC or VTA according to the coordinates of Paxinos and Watson (Paxinos and Watson, 1986). Coordinates from bregma with flat skull were: mPFC, AP +3.0 mm, DV -3.0 mm, ML +0.6 mm; VTA, AP -5.95 mm, DV -7.3 mm, ML +0.9 mm. Three-five $d$ thereafter, the jugular vein was cannulated under xylazine-ketamine; animals recovered for $3 \mathrm{~d}$ before nicotine self-administration began, while animals used in the sucrose SA experiment recovered for 7 days after stereotaxic surgery before the onset of sucrose SA training. All procedures were conducted in accordance with NIH Guidelines concerning the Care and Use of Laboratory Animals and were approved by the Animal Care and Use Committee of the University of Tennessee Health Science Center. 


\subsubsection{Procedures for chronic nicotine self-administration and extinction followed by microdialysis}

Nicotine self-administration was performed according to our protocols published previously (Valentine et al., 1997; Wang et al., 2007). Briefly, immediately following acute surgical recovery, animals were placed into operant chambers where they resided for the duration of the study. After a $3 \mathrm{~d}$ recovery interval, rats were randomly assigned to treatment groups and jugular lines were filled with either $1 \mathrm{mM}$ nicotine in $200 \mathrm{U} / \mathrm{ml}$ heparinized saline or heparin saline alone. The operant chamber contained two levers positioned $5 \mathrm{~cm}$ above the floor; a green cue light $1 \mathrm{~cm}$ above each lever signaled the availability of nicotine. One lever was randomly assigned as the active lever, which triggered the pump to deliver one $50 \mu \mathrm{l}$ bolus injection of nicotine over $0.81 \mathrm{sec}$ per 300 g body weight $(0.03 \mathrm{mg} / \mathrm{kg})$ or saline; pressing the inactive lever had no consequence. Without prior training, shaping or food deprivation, rats were allowed to acquire and maintain self-administration behavior throughout consecutive $23 \mathrm{~h}$ sessions for $18 \mathrm{~d}$, and a microdialysis experiment began on d 19. Specifically, the animals were removed from their operant chamber at 9 A.M. and transferred to the microdialysis apparatus approximately $3 \mathrm{~h}$ before the first baseline sample was collected.

A second microdialysis experiment was conducted after $10 \mathrm{~d}$ of extinction from nicotine SA. In this study, after $18 \mathrm{~d}$ of nicotine SA the $10 \mathrm{~d}$ extinction phase was initiated; pressing the active lever had no programmed consequence and the cue lights were not illuminated. On d 11 of extinction, a microdialysis experiment was performed on a group of rats that were naïve to microdialysis.

\subsubsection{In vivo microdialysis procedure}

Concentric microdialysis probes (1.5 $\mathrm{mm}$ for VTA, $2.0 \mathrm{~mm}$ for $\mathrm{mPFC}$; MW cutoff 13,000 Da, outer diameter $235 \mu \mathrm{m}$ ) were constructed in our laboratory, as reported previously (Fu et al., 2000). The recovery efficiencies for glutamate by $1.5 \mathrm{~mm}$ and 2.0 $\mathrm{mm}$ probes were $4.7 \pm 0.5 \%(\mathrm{n}=4)$ and $7.5 \pm 0.6 \%(\mathrm{n}=5)$, respectively, and for GABA by $1.5 \mathrm{~mm}$ and $2.0 \mathrm{~mm}$ probes were $6.6 \pm 0.6 \%(\mathrm{n}=3)$ and $8.7 \pm 0.4 \%(\mathrm{n}=3)$, respectively. Microdialysis was performed as described previously (Fu et al., 2000). Briefly, on d 19 at 9:00 A.M. during their active (dark) phase, rats were moved into the alert-rat microdialysis chambers (CMA, Chelmsford, MA) located within a dark isolation room, lit by a red safe-light; microdialysis probes were inserted into both $\mathrm{mPFC}$ and VTA guide cannulae. Following insertion, probes were perfused ( $2 \mu 1 / \mathrm{min})$ with Kreb's Ringer Buffer (KRB; $147 \mathrm{mM} \mathrm{NaCl}, 4.0 \mathrm{mM} \mathrm{KCl}$, and $3.4 \mathrm{mM} \mathrm{CaCl}_{2}$ in polished water; $0.2 \mu \mathrm{m}$ filter sterilized and degassed) for $2 \mathrm{~h}$. Thereafter, 20-min microdialysate samples were collected into plastic vials; three consecutive samples were collected to measure basal glutamate and GABA levels prior to drug administration. mPFC was sequentially perfused with the following solutions, each for $1 \mathrm{~h}$ : KRB, $200 \mu \mathrm{M}$ NMDA, KRB, 500 $\mu \mathrm{M}$ NMDA, KRB, $100 \mathrm{mM} \mathrm{KCl}$. 
At the end of each experiment, probe positions were verified by histological examination. Only data obtained from animals with probes in the correct position were used for analysis.

\subsubsection{HPLC-electrochemical analysis}

Mobile phase (100 mM sodium dihydrogen phosphate monohydrate in $15 \%$ methanol $\mathrm{v} / \mathrm{v}$ in polished water, $\mathrm{pH} 4.4$ with phosphoric acid) was perfused through a reverse-phase column (4.6 x 80 mm, $3 \mu \mathrm{m}$; model HR-80; ESA Inc., Chelmsford, USA) at $1.5 \mathrm{ml} / \mathrm{min}$, using an ESA Model 582 solvent delivery module. O-phthalaldehyde (OPA)/sulfite stock solution was made by dissolving $22 \mathrm{mg}$ of OPA in $0.5 \mathrm{ml}$ of ethanol, and then adding $0.5 \mathrm{ml}$ of $1 \mathrm{M}$ sodium sulfite and $9 \mathrm{ml}$ of $0.1 \mathrm{M}$ sodium tetraborate, $\mathrm{pH}$ 10.0. The working OPA/sulfite solution was prepared daily by diluting the stock OPA/sulfite solution with polished water. Glutamate and GABA were automatically derivatized, samples were maintained at $12^{\circ} \mathrm{C}$, and $27 \mu \mathrm{L}$ was injected on column by an ESA 542 autosampler. Electrochemical detection was performed at $-400 \mathrm{mV}$ (reduction electrode 1) and $+200 \mathrm{mV}$ (reduction electrode 2) with gain set at $200 \mathrm{nA}$ and guard cell at $350 \mathrm{mV}$. Similar to a previous report from our laboratory, the limits of detection for glutamate and GABA were $25 \mathrm{pg} /$ injection and $0.78 \mathrm{pg} /$ injection, respectively (Fu et al., 2000). Serially diluted standards containing known quantities of glutamate and GABA were included in each assay $\left(\mathrm{R}^{2}>0.98\right)$.

\subsubsection{Brain punches and tissue preparation}

On d 11 of extinction from chronic nicotine SA, animals were sacrificed to obtain brain tissues for western immunoblotting of ionotropic glutamate receptors. The brain punching procedure and tissue preparation were done according to an established protocol (Wang et al., 2007). Briefly, rats were anesthetized with isoflurane, decapitated, and brains were removed and stored at $-80{ }^{\circ} \mathrm{C}$. Frozen brain sections, $0.7 \mathrm{~mm}$ thickness, were obtained using arrays of 20 double-edge razor blades, designed and assembled in our laboratory. The mPFC and VTA were punched out according to the atlas of Paxinos and Watson (Paxinos and Watson, 1986) using a 20 gauge syringe adapter. Dissected tissues were immediately transferred to tubes embedded in dry ice and stored at $-80{ }^{\circ} \mathrm{C}$ until further processing. Dissected tissues were sonicated in $2 \%$ sodium dodecyl sulfate (SDS) solution for $30 \mathrm{sec}$ in an ice-water bath. Sonicated tissues were centrifuged at $800 \times \mathrm{g}, 5 \mathrm{~min}$. Protein concentrations were measured using the bicinchoninic acid assay (Pierce, Rockford, IL) with bovine serum albumin (BSA) as the standard.

\subsubsection{Western immunoblotting}

To determine whether the up-regulated expression of ionotropic glutamate receptors observed during chronic nicotine SA (Wang et al., 2007) persisted after $10 \mathrm{~d}$ of extinction, western immunoblotting was performed following the protocol published 
previously (Wang et al., 2007). Briefly, prepared tissue samples were run on $10 \%$ polyacrylamide gels and then transferred to PVDF membrane (Bio-Rad, Hercules, CA) at $4^{\circ} \mathrm{C}$ overnight. Membranes were blocked by $5 \%$ non-fat dried milk for $2 \mathrm{~h}$. Based on the molecular weight differences between NMDA receptor subunits, AMPA receptor subunits, and $\beta$ - actin, blots were carefully cut into three separate sections by visualizing the molecular weight markers: upper blots were used to detect NMDA receptor subunits, middle blots to detect AMPA receptor subunits, and lower blots to detect $\beta$-actin in order to evaluate protein loading. Blots then were incubated with the following antibodies: mouse anti-beta actin (1:1000; Sigma, St. Louis, MO), rabbit anti-glutamate receptor subtype 1 (GluR1; 1:1000; Upstate, Lake Placid, NY), rabbit anti-GluR 2/3 (1:1000; Upstate, Lake Placid, NY), goat anti-NMDA receptor subtype 1 (NR1; 1:400; Santa Cruz, CA) or goat anti-NR 2A (1:200; Santa Cruz) or rabbit anti-NR 2B (1:500; Upstate). Blots were then washed in TBST and incubated in HRP-conjugated secondary antibodies (1:3000 for beta actin, 1:1500 for all others). All antibodies were diluted in the same blocking solution and incubated for $2 \mathrm{~h}$ at room temperature or overnight at $4^{\circ} \mathrm{C}$. Target proteins were visualized by Supersignal (Pierce, Rockford, IL) followed by imaging using a Biorad ChemiDoc with quantitation by Quantity One (Bio-Rad, Hercules, CA).

\subsubsection{Sucrose self-administration}

In two separate experiments, we examined the effects of blocking mPFC NMDA receptors or VTA AMPA receptors on sucrose self-administration. Beginning $7 \mathrm{~d}$ after stereotaxic implantation of microinjection guide cannulae into mPFC or VTA, rats were food-deprived to $18 \mathrm{~g}$ of standard food pellets per $\mathrm{d}$ for the duration of the experiment and the first training session, using sucrose pellet reinforcement, was initiated $2 \mathrm{~d}$, thereafter. In each training session, rats were placed in an operant chamber for no longer than 60 min or until 100 sucrose pellets were obtained on an FR1 schedule. The operant chamber consisted of two levers positioned $7 \mathrm{~cm}$ above the floor; a yellow cue light $1 \mathrm{~cm}$ above each lever signaled the availability of sucrose pellets (45 mg, Research Diets, New Brunswick, NJ). One lever, randomly assigned as active, triggered the delivery of a sucrose pellet; pressing the inactive lever had no programmed consequence. The delivery of each sucrose pellet was followed by a $10 \mathrm{sec}$ time-out during which the yellow light above the active bar was not illuminated and sucrose pellets were not available. The animals acquired the behavior by d $10 \pm 0.97$. The sucrose-training phase continued for a total of $18 \mathrm{~d}$, in parallel to the number of days that animals self-administered nicotine in other experiments. On d 19, two separate experiments were conducted to determine the effects of the NMDA or AMPA receptor antagonists, AP-5 or NBQX, on sucrose reinforced operant behavior. 


\subsubsection{Intraparenchymal microinjections after chronic nicotine self-administration or sucrose self-administration}

We examined the effects of blocking either mPFC NMDA receptors or VTA AMPA receptors in separate groups of rats that self-administered either nicotine or sucrose. Rats were acclimated to the stress of brain microinjection by handling for $3 \mathrm{~min}$ prior to the start of each sucrose SA training or nicotine SA session. After $18 \mathrm{~d}$ of sucrose or nicotine SA, rats received microinjections in the mPFC or VTA on d 19,21 and 23, 10 min prior to the test session; they self-administered nicotine or sucrose on the intervening days. On the 3 test days, each rat that self-administered either nicotine or sucrose received a microinjection $(500 \mathrm{nl} / 120 \mathrm{sec})$ of KRB or AP-5 $(1.5 \mu \mathrm{g}$ or $5 \mu \mathrm{g}$, bilaterally) into the $\mathrm{mPFC}$ in a counterbalanced order. Similarly, separate groups of rats received microinjections of KRB or NBQX $(0.067 \mu \mathrm{g}$ or $0.25 \mu \mathrm{g}$, bilaterally) into the VTA.

To assess the efficacy of administering AP-5 into the $\mathrm{mPFC}$, a third microdialysis study was performed. Animals were randomly assigned to 2 groups in which either NMDA alone or AP-5 and NMDA (volumes and rates of delivery as previously defined) was microinjected into $\mathrm{mPFC}$. Beginning $10 \mathrm{~min}$ thereafter, VTA microdialysates were collected at 20 min intervals for measurements of glutamate.

\subsubsection{Locomotion}

To determine whether an intra-VTA microinjection of NBQX impaired movement, we evaluated the effects of intra-VTA NBQX on locomotion. Locomotor activity was measured by a Digiscan Micro Monitoring system (Omni-tech Electronics, Columbus, Ohio). This system consists of an activity box $(45 \times 24 \times 19 \mathrm{~cm})$ placed on a sensor-equipped aluminum frame within a sound-attenuating chamber. The frames contain 16 light beams and detectors, linked to a computer via a micro analyzer (Accuscan Instruments Inc., Columbus, Ohio). Movement is detected by a photocell when the animal interrupts an individual light beam. One day before an actual experiment, each animal was adapted to the behavioral chamber and injection procedure for 120 minutes. On experimental days 1, 3 and 5, when locomotor activity data were collected, each animal was placed in a test chamber $1 \mathrm{~h}$ before receiving a microinjection. Following injections, locomotor activity was recorded in $15 \mathrm{~min}$ bins for $2 \mathrm{~h}$.

\subsubsection{Data analysis}

To determine the specificity of nicotine SA, the number of daily active and inactive lever presses were compared within each treatment group (i.e., nicotine and saline) and between groups by two-way analysis of variance (ANOVA; SPSS 13.0, SPSS Inc., Chicago, IL). Data are expressed as mean \pm SEM.

For microdialysis experiments, the peak heights of each analyte were analyzed by PowerChrom (ADInstruments, Castle Hill, Australia) and concentrations were 
determined by interpolation of sample peak heights relative to standard curves. In each animal, the basal glutamate or GABA value was defined as the average of the three samples collected immediately prior to the administration of NMDA. Group data (mean \pm SEM) were expressed as a multiple of the basal glutamate or GABA level that was determined for each animal. All statistical analyses of glutamate and GABA levels were performed by two-way ANOVA with repeated measures (SPSS 13.0; SPSS Inc., Chicago, IL) with the exception of analysis of peak incremental responses, which used one-way ANOVA.

For western immunoblotting, each sample from a rat was run once on each of two gels. Optical density values for each lane were normalized to $\beta$-actin in order to control for variation in loading and transfer. For each sample, normalized values that had been averaged between the two blots were used to conduct statistical comparisons (unpaired student t-test) to identify treatment effects within a region for each subunit. The mean values of nicotine treatment groups were expressed as a percentage of saline \pm SEM. Treatment differences were considered significant at $\mathrm{p}<0.05$.

Lastly, the effects of AP-5 or NBQX on chronic nicotine or sucrose SA were examined by one-way ANOVA, followed by Fisher's LSD post hoc tests to detect differences between vehicle and drug effects. The effects of NBQX on locomotion were analyzed by two-way ANOVA with repeated measures.

\subsection{Results}

\subsubsection{Chronic Nicotine SA and extinction from nicotine SA}

Figure 3.1A shows the acquisition and maintenance of chronic nicotine SA by rats that were microdialyzed on d19. From 44 onward, active lever presses (FR1) in the nicotine SA group were approximately 3-4-fold higher than inactive presses (two-way ANOVA with repeated measures: $\mathrm{F}_{1,10}=38.89, \mathrm{p}<0.001$ ). In addition, the number of active lever presses was greater in the nicotine $S A$ than saline group $\left(\mathrm{F}_{1,10}=7.21, \mathrm{p}<0.05\right)$. There was no difference in active $v s$ inactive lever presses in the saline group $\left(\mathrm{F}_{1,10}=0.31\right.$, $\mathrm{p}>0.05)$.

The SA profiles of rats that were microdialyzed after extinction from chronic nicotine SA are shown in Figure 3.1B. Similar to panel A, during the first $18 \mathrm{~d}$, active lever presses were greater than inactive in the nicotine $S A$ group $\left(F_{1,6}=13.78, p<0.05\right)$, whereas no difference was observed in the saline group $\left(\mathrm{F}_{1,6}=0.22, \mathrm{p}>0.05\right)$. During the $10 \mathrm{~d}$ extinction period, active lever presses were no greater in the nicotine group compared to saline $\left(\mathrm{F}_{1,6}=3.71, \mathrm{p}>0.05\right)$. Furthermore, comparing the final $3 \mathrm{~d}$ of the extinction phase to that during nicotine $\mathrm{SA}$, the mean number of active lever presses were less (extinction vs nicotine SA: $23.9 \pm \pm 5.2$ vs $53.8 \pm 6.8$ active lever presses; $\left.\mathrm{F}_{1,6}=12.11, \mathrm{p}<0.05\right)$. 

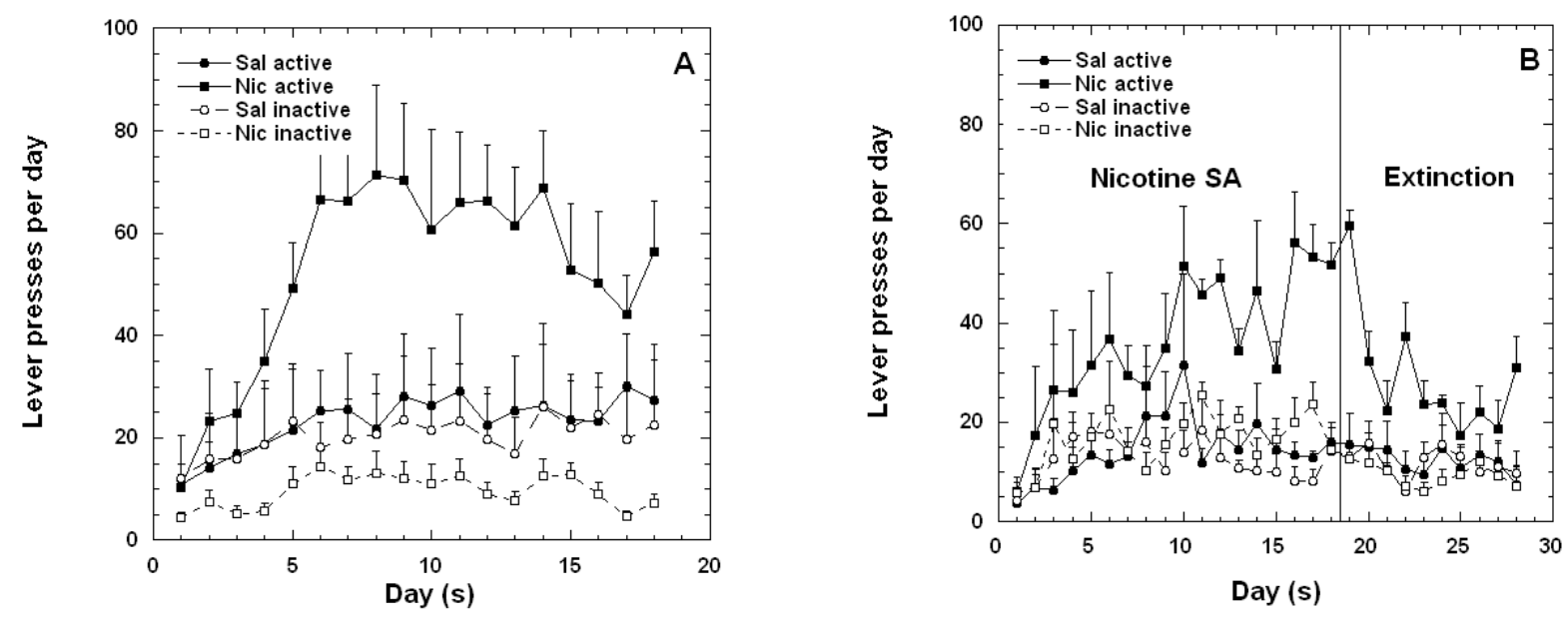

Figure 3.1. Lever press activity during chronic nicotine self-administration (SA) and extinction. (Panel A) Adult male Lewis rats acquired nicotine SA $(0.03 \mathrm{mg} / \mathrm{kg}$ b.wt./injection, iv) without prior training, priming or food deprivation when the drug was available $23 \mathrm{~h} / \mathrm{d}$ from d 1-18. Two way ANOVA with repeated measures revealed that active lever presses were greater than inactive presses in the nicotine (Nic) group $\left(\mathrm{F}_{1,10}=38.89, \mathrm{p}<0.001 ; \mathrm{n}=7\right)$, but not in the saline (Sal) group $\left(\mathrm{F}_{1,10}=0.31, \mathrm{p}>0.05 ; \mathrm{n}=5\right)$. Comparing treatment groups, active lever presses were greater in the Nic than Sal group $\left(F_{1,10}=6.79, p<0.05\right)$. (Panel B) A separate cohort of rats self-administered Nic or Sal for $18 \mathrm{~d}$, followed by $10 \mathrm{~d}$ of extinction. Two way ANOVA with repeated measures showed that, after extinction, active lever presses in the Nic group $(\mathrm{n}=4)$ were no longer different from the $\mathrm{Sal}(\mathrm{n}=4)$ group $\left(\mathrm{F}_{1,6}=3.71, \mathrm{p}>0.05\right)$. In addition, the mean number of active lever presses during the last $3 \mathrm{~d}$ of the late maintenance phase (d 16-18) were greater than during the final $3 \mathrm{~d}$ of the extinction period (e.g., $\mathrm{d} 26-28)\left(\mathrm{F}_{1,6}=12.11, \mathrm{p}>0.05\right)$. Data are expressed as mean $\pm \mathrm{SEM}$. 


\section{2. $\mathrm{mPFC}$ and VTA glutamate responsiveness to intra-mPFC NMDA during chronic nicotine SA}

Histological evaluation was performed to determine whether the membrane region of microdialysis probes and the tip of microinjection cannulae were within $\mathrm{mPFC}$ and VTA. Figure 3.2 shows the neuroanatomical location of these probes and cannulae from rats that were included in the data analyses.

On d 19 of nicotine and saline SA, two sequential doses of NMDA were administered via the $\mathrm{mPFC}$ microdialysis probe during dual probe (mPFC and VTA) microdialysis of freely moving rats. Chronic nicotine SA did not affect basal glutamate levels in the mPFC $(108.4 \pm 16.1$ vs $93.1 \pm 17.9 \mathrm{pg} / \mu 1$ for nicotine and saline SA, respectively; $\mathrm{p}>0.05)$ or VTA $(21.7 \pm 4.1$ s $20.9 \pm 3.7 \mathrm{pg} / \mu \mathrm{l}$ for nicotine and saline SA, respectively; $\mathrm{p}>0.05$ ). Figure $3.3 \mathrm{~A}$ shows that nicotine $\mathrm{SA}$ enhanced the $\mathrm{mPFC}$ glutamate release induced by $200 \mu \mathrm{M}$ NMDA (two-way ANOVA: $\mathrm{F}_{1,10}=6.76, \mathrm{p}<0.05$ ), but not by $500 \mu \mathrm{M}$ NMDA $\left(\mathrm{F}_{1,10}=0.91, \mathrm{p}>0.05\right)$. Further analysis of the same data, expressed as peak incremental glutamate responses (Figure 3.3B), showed that both nicotine SA and the dose of NMDA affected glutamate release (two-way ANOVA: $F_{1,10}=15.77, p<0.01$ and $\mathrm{F}_{1,10}=31.13, \mathrm{p}<0.001$, respectively). Additionally, nicotine SA amplified the effects of both $200 \mu \mathrm{M}$ and $500 \mu \mathrm{M}$ NMDA (one-way ANOVAs: $\mathrm{F}_{1,10}=9.71, \mathrm{p}<0.05$ and $\mathrm{F}_{1,10}=8.74, \mathrm{p}<0.05$, respectively). In contrast, the mPFC glutamate response to $100 \mathrm{mM}$ $\mathrm{KCl}$ was not affected by nicotine $\mathrm{SA}\left(\mathrm{F}_{1,10}=0.99, \mathrm{p}>0.05\right)$.

Figure 3.3C, shows the time course of glutamate release in the VTA in response to different concentrations of intra-mPFC NMDA or $100 \mathrm{mM} \mathrm{KCl}$ on d 19 of chronic nicotine SA. Nicotine SA amplified the release of VTA glutamate stimulated by $200 \mu \mathrm{M}$ and $500 \mu \mathrm{M}$ NMDA (two-way ANOVAs: $\mathrm{F}_{1,10}=6.36, \mathrm{p}<0.05$ and $\mathrm{F}_{1,10}=5.96, \mathrm{p}<0.05$, respectively). The peak incremental VTA glutamate responses (Figure 3.3D) were affected by nicotine $\mathrm{SA}$ and the dose of NMDA $\left(\mathrm{F}_{1,9}=32.21, \mathrm{p}<0.001\right.$ and $\mathrm{F}_{1,9}=18.73$, $\mathrm{p}<0.01$, respectively), but there was no interaction between these variables $\left(\mathrm{F}_{1,9}=2.33\right.$, $\mathrm{p}>0.05$ ). Additionally, the peak glutamate responses to $200 \mu \mathrm{M}$ and $500 \mu \mathrm{M}$ NMDA were both increased by nicotine SA (one-way ANOVAs: $200 \mu \mathrm{M}$ NMDA: $F_{1,9}=16.99$, $\mathrm{p}<0.01 ; 500 \mu \mathrm{M}$ NMDA: $\left.\mathrm{F}_{1,8}=16.67, \mathrm{p}<0.01\right)$. Lastly, nicotine SA did not affect the peak incremental glutamate response to $100 \mathrm{mM} \mathrm{KCl}\left(\mathrm{F}_{1,9}=0.17, \mathrm{p}>0.05\right)$. Therefore, chronic nicotine SA augmented glutamate responsiveness to NMDA in MPFC and VTA without affecting basal levels of glutamate in these regions.

\subsection{3. $\mathrm{mPFC}$ and VTA glutamate responsiveness to intra-mPFC NMDA after extinction from chronic nicotine $\mathrm{SA}$}

On $\mathrm{d} 11$ following the cessation of nicotine SA, animals were microdialyzed to assess the persistence of glutamate hyper-responsiveness to intra-mPFC NMDA. In both $\mathrm{mPFC}$ andVTA, basal glutamate levels were unaffected by prior nicotine SA (nicotine $v s$ saline SA: $102.0 \pm 18.8$ vs. $93.8 \pm 15.9 \mathrm{pg} / \mu \mathrm{l}$ in $\mathrm{mPFC}$ and $27.8 \pm 6.0$ vs. $34.0 \pm 10.0$ $\mathrm{pg} / \mu \mathrm{l}$ in VTA; $\mathrm{p}>0.05$ for both comparisons). 


\section{MPFC}

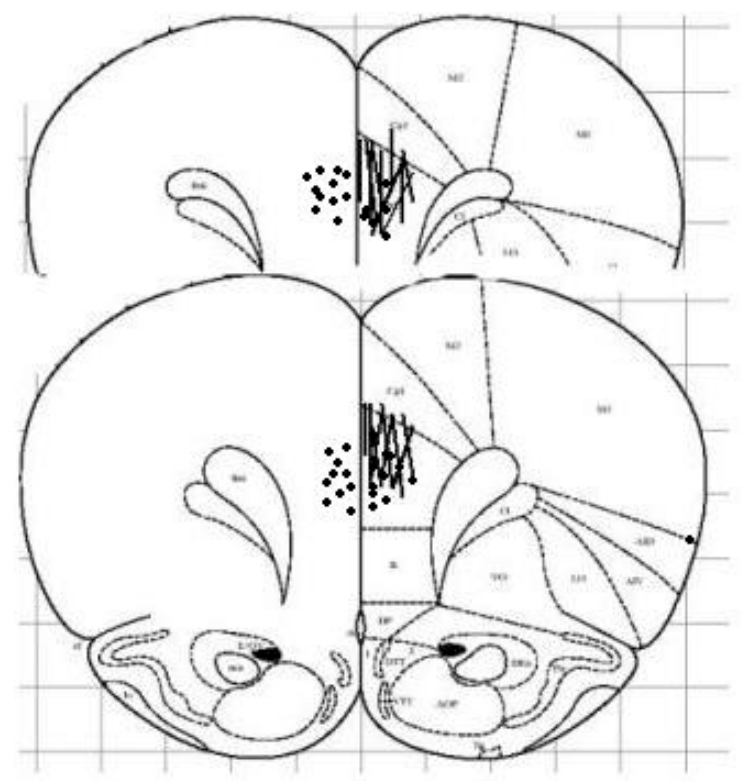

VTA

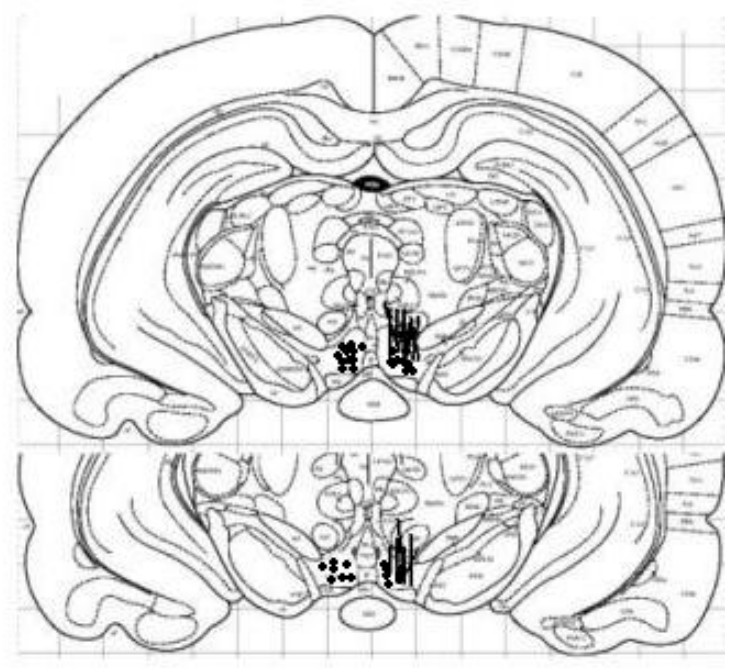

Figure 3.2. Schematic representation of the positions of all microdialysis probes and microinjection cannulae in the rat $\mathrm{mPFC}$ and VTA. Coronal brain sections were analyzed to verify the position of the membrane segment of each microdialysis probe and microinjection cannulae (the membrane regions of probes are indicated by line segments and the tips of microinjection cannulae by dots). All animals used in these investigations are represented. $\mathrm{mPFC}$ and VTA diagrams are $3.0 \mathrm{~mm}$ anterior and $5.95 \mathrm{~mm}$ posterior to bregma, respectively (adapted from Paxinos and Watson, 1986). 

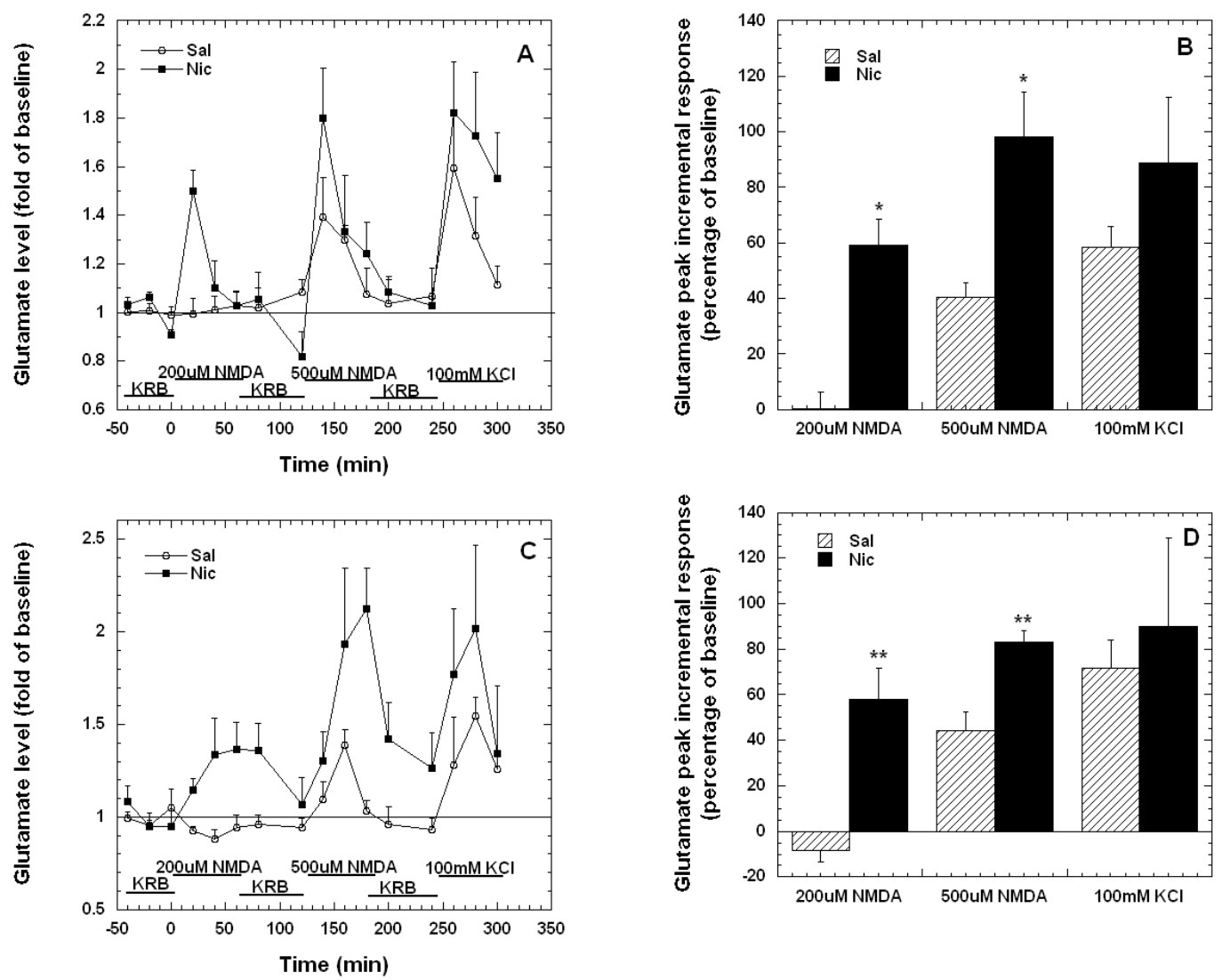

Figure 3.3. Chronic nicotine $\mathrm{SA}$ enhanced glutamate release in the $\mathrm{mPFC}$ and VTA induced by intra-mPFC NMDA (administered by reverse microdialysis). Glutamate was measured in microdialysates obtained concurrently from both brain regions. Basal levels of glutamate in $\mathrm{mPFC}$ and VTA were unaffected by nicotine SA. (Panel A) In $\mathrm{mPFC}$, glutamate responses to NMDA $(200 \mu \mathrm{M}$ and $500 \mu \mathrm{M})$ and $100 \mathrm{mM} \mathrm{KCl}$ were determined on d 19 of SA. Two-way ANOVA with repeated measures revealed that nicotine SA (Nic; $n=7)$ amplified the glutamate response to $200 \mu \mathrm{M}$ NMDA in comparison to saline $\mathrm{SA}(\mathrm{Sal} ; \mathrm{n}=5)\left(\mathrm{F}_{1,10}=6.76, \mathrm{p}<0.05\right)$. (Panel B) In $\mathrm{mPFC}$, the glutamate peak incremental responses were calculated from the data in panel A. One-way ANOVAs indicated that nicotine SA increased the peak incremental response induced by each dose of NMDA ( $200 \mu \mathrm{M}$ NMDA, $\mathrm{F}_{1,10}=9.71, \mathrm{p}<0.05 ; 500 \mu \mathrm{M}$ NMDA, $\mathrm{F}_{1,10}=8.74$, $\mathrm{p}<0.05$ ). (Panel C) In VTA, the glutamate responses to NMDA and $\mathrm{KCl}$ were determined on d 19 of SA. Nicotine SA (n=7) amplified the glutamate responses to $200 \mu \mathrm{M}$ and 500 $\mu \mathrm{M}$ NMDA $(\mathrm{n}=5)$ (two-way ANOVAs: $\mathrm{F}_{1,10}=6.36$, $\mathrm{p}<0.05$ for $200 \mu \mathrm{M}$ NMDA; $\mathrm{F}_{1,10}=5.96, \mathrm{p}<0.05$ for500 $\mu \mathrm{M}$ NMDA). (Panel D) In VTA, the glutamate peak incremental responses were calculated from the data in panel C. NMDA dosedependently stimulated glutamate release (two-way ANOVAs: $\mathrm{F}_{1,9}=18.73, \mathrm{p}<0.01$ ). The glutamate responses to both doses of NMDA were enhanced by nicotine SA (one-way ANOVAs: $200 \mu \mathrm{M}$ NMDA, $F_{1,9}=16.99, \mathrm{p}<0.01 ; 500 \mu \mathrm{M} \mathrm{NMDA}_{1,9}=16.67, \mathrm{p}<0.01$ ). Data are expressed as mean \pm SEM. ${ }^{*}, \mathrm{p}<0.05$; **, $\mathrm{p}<0.01$ comparing Nic vs. Sal SA at the same dose of NMDA. 
On 11 following the extinction of nicotine SA, Figure 3.4A shows that NMDAinduced glutamate release in $\mathrm{mPFC}$ was no longer enhanced by the prior selfadministration of nicotine. $\left(\mathrm{F}_{1,6}=0.50, \mathrm{p}>0.05\right)$. Additionally, the glutamate response was greater to $500 \mu \mathrm{M}$ than $200 \mu \mathrm{M}$ NMDA $\left(\mathrm{F}_{1,6}=16.91, \mathrm{p}<0.01\right)$. Similarly, in the VTA, Figure 3.4B shows the persistence of dose-dependent NMDA-induced glutamate release $\left(\mathrm{F}_{1,6}=18.86, \mathrm{p}<0.01\right)$ and the absence of enhancement by prior nicotine $\mathrm{SA}\left(\mathrm{F}_{1,6}=0.02\right.$, $\mathrm{p}>0.05)$. Hence, glutamate hyper-responsiveness was no longer detected in $\mathrm{mPFC}$ or VTA by d 11 after cessation of chronic nicotine SA.

\subsection{4. $\mathrm{mPFC}$ and VTA GABA responsiveness to intra-mPFC NMDA during chronic nicotine $\mathrm{SA}$}

GABA levels were measured to evaluate whether nicotine SA altered GABA levels in association with the foregoing changes in glutamate release. Chronic nicotine SA did not affect basal GABA levels in the mPFC or VTA (nicotine $v s$ saline SA: $1.1 \pm 0.3 v s 1.5 \pm 0.3 \mathrm{pg} / \mu \mathrm{l}$ in $\mathrm{mPFC}, \mathrm{p}>0.05 ; 1.2 \pm 0.3 v s 1.7 \pm 0.2 \mathrm{pg} / \mu \mathrm{l}$ in VTA, $\mathrm{p}>0.05$ ). Figure $3.5 \mathrm{~A}$ shows that mPFC GABA levels were stimulated by 200 and 500 $\mu$ M NMDA (200 $\mu$ M NMDA: $F_{1,14}=17.41, p<0.01 ; 500 \mu$ M NDA: $\left.F_{1,14}=6.18, p<0.05\right)$, but were not affected by nicotine $\mathrm{SA}\left(\mathrm{F}_{1,6}=0.45, \mathrm{p}>0.05\right)$ (data were combined from both treatment groups for analysis of NMDA effects in Figs. 3.5A,B). In contrast, VTA GABA (Figure 3.5B) was not increased by intra-mPFC NMDA (200 $\mu$ M NMDA: $\mathrm{F}_{1,10}=2.18, \mathrm{p}>0.05 ; 500 \mu \mathrm{M}$ NMDA: $\left.\mathrm{F}_{1,10}=3.09, \mathrm{p}>0.05\right)$. Intra-mPFC $\mathrm{KCl}$ stimulated GABA release in both regions $\left(\mathrm{F}_{1,14}=32.87, \mathrm{p}<0.001\right.$ for $\mathrm{mPFC} ; \mathrm{F}_{1,10}=19.56, \mathrm{p}<0.01$ for VTA); the data showing a 20 -fold increase is not illustrated in Figure 3.5A. In summary, nicotine SA had no effect on basal or NMDA induced GABA release in the mPFC or VTA.

\subsubsection{Expression of ionotropic glutamate receptor subunits in the mPFC and VTA after extinction from nicotine $\mathrm{SA}$}

We have previously reported that specific ionotropic glutamate receptor subunits, in $\mathrm{mPFC}$ and VTA, are up-regulated by chronic nicotine SA (Wang et al., 2007). To further evaluate the association between glutamate hyper-responsiveness and receptor subunit expression, these specific subunits were quantified by western immunoblotting of mPFC and VTA extracts obtained from animals after $10 \mathrm{~d}$ of extinction from nicotine SA. Representative gels from each treatment group are shown in Figure 3.6. NMDA receptor NR2A and NR2B subunits were both visualized as single bands at approximately $180 \mathrm{kDa}$. In contrast to the up-regulation of these subunits in $\mathrm{mPFC}$ during nicotine SA (Wang et al., 2007), after $10 \mathrm{~d}$ of extinction this was not longer evident (Figure 3.6A). The GluR1 and GluR2/3 subunits in VTA were each single bands visualized at $106 \mathrm{kDa}$ and $110 \mathrm{kDa}$, respectively (Figure 3.6B). The up-regulated expression of GluR2/3, previously reported during nicotine SA(Wang et al., 2007), did not persist after $10 \mathrm{~d}$ of extinction. Therefore, after extinction from chronic nicotine 

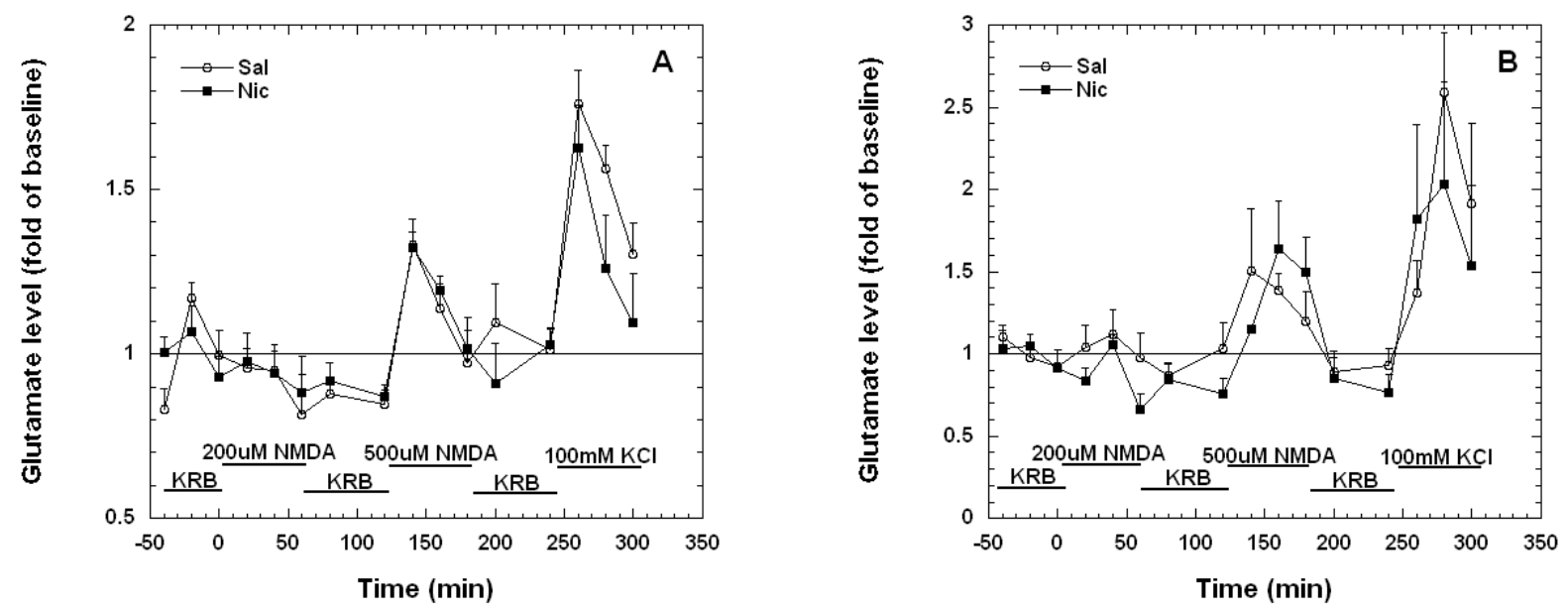

Figure 3.4. After extinction from chronic nicotine $\mathrm{SA}$, glutamate release in the mPFC (panel A) and VTA (panel B), induced by intra-mPFC NMDA, was not enhanced by prior nicotine SA. Glutamate was measured in concurrent microdialysates obtained from both brain regions. Basal levels of glutamate in $\mathrm{MPFC}$ and VTA were unaffected by prior nicotine SA. After $10 \mathrm{~d}$ of extinction, two way ANOVA with repeated measures indicated that both the $\mathrm{mPFC}$ and VTA glutamate responses induced by intra-mPFC NMDA ( 200 or $500 \mu \mathrm{M}$; administered by reverse microdialysis) or $\mathrm{KCl}$ were not affected by prior nicotine $\mathrm{SA}\left[\mathrm{mPFC}\right.$ (panel A): $\mathrm{F}_{1,6}=0.50, \mathrm{p}>0.05$; VTA (panel B): $\mathrm{F}_{1,6}=0.02, \mathrm{p}>0.05 ; \mathrm{n}=4$ for Nic and Sal]. 

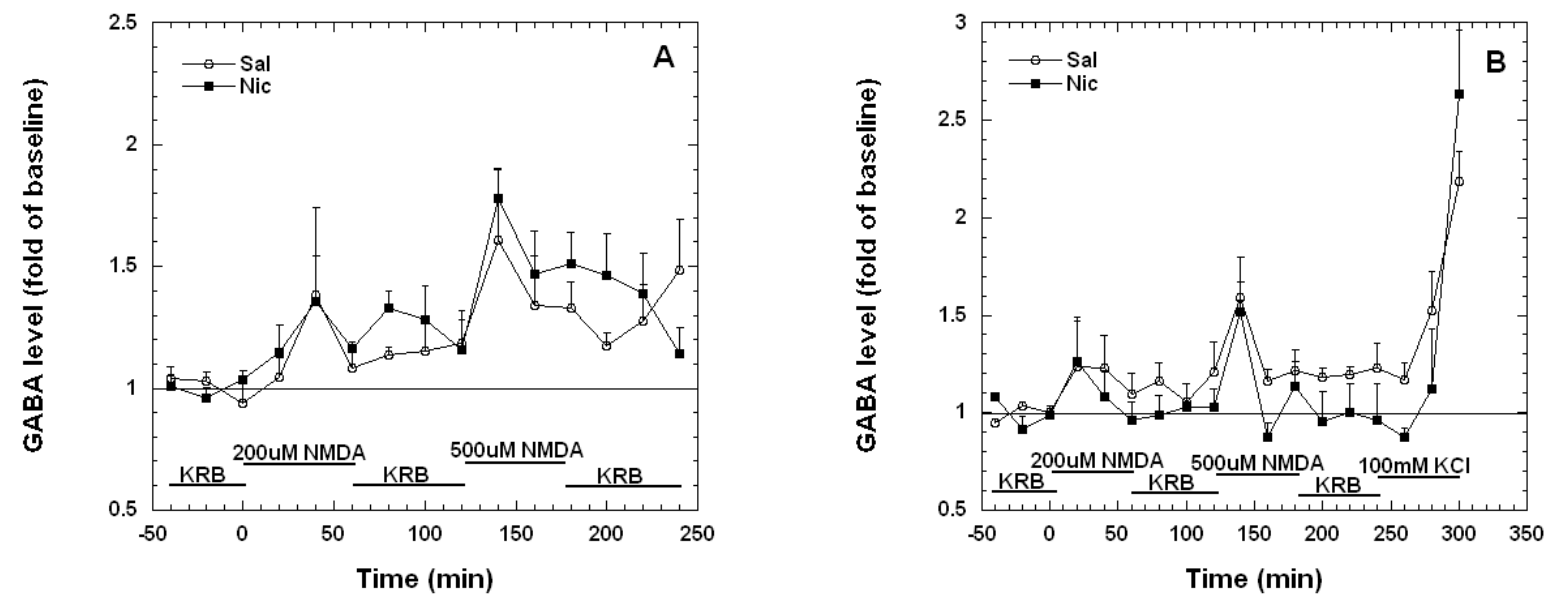

Figure 3.5. GABA responses in the mPFC (panel A) and VTA (panel B), induced by intra-mPFC NMDA, were not enhanced by prior nicotine SA. GABA levels were measured on the microdialysates obtained in the experiments shown in Figure 3. Two way ANOVA with repeated measures showed that the $\mathrm{mPFC}$ and VTA GABA responses to 200 or $500 \mu \mathrm{M}$ NMDA were not affected by nicotine SA [mPFC (panel A): $\mathrm{F}_{1,6}=0.45$, $\mathrm{p}>0.05$; VTA (panel B): $\mathrm{F}_{1,4}=0.51, \mathrm{p}>0.05 ; \mathrm{n}=3-5$ ). 

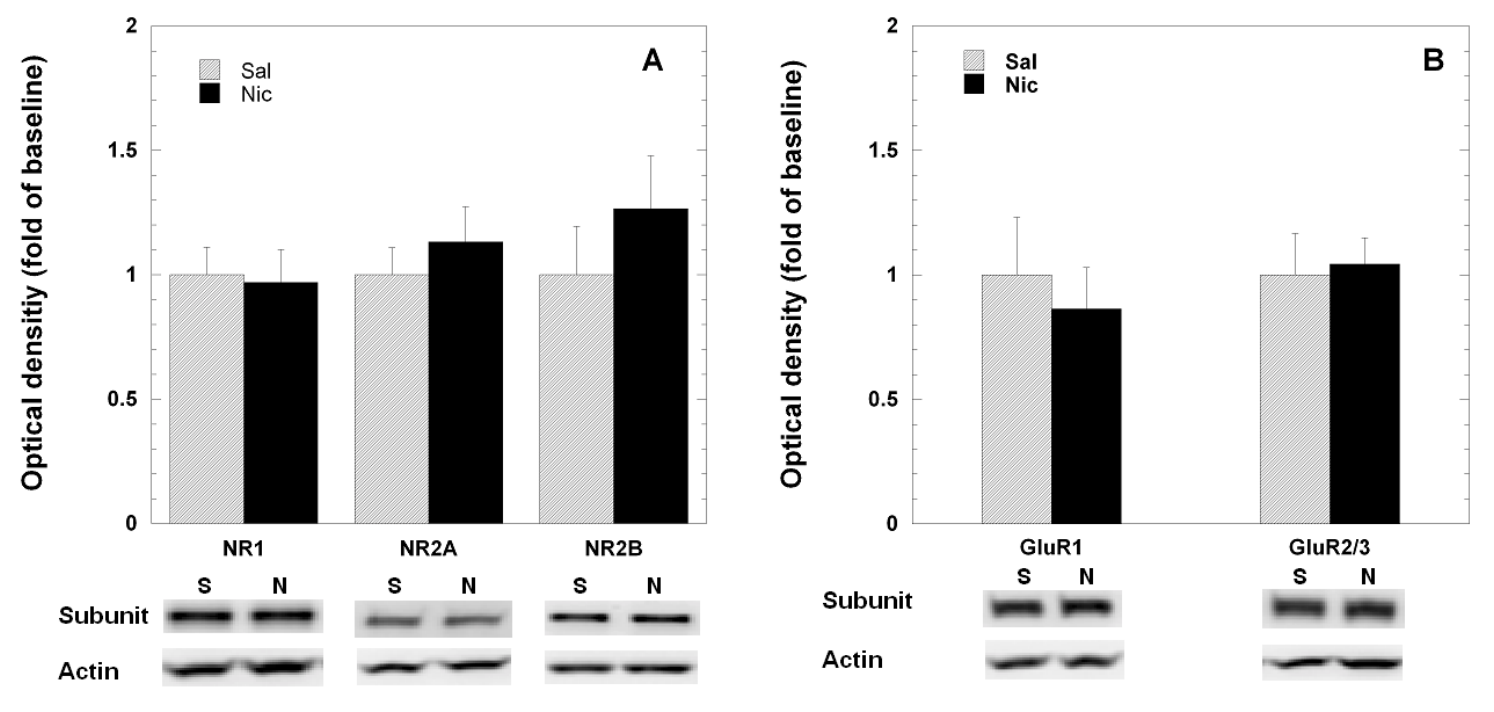

Figure 3.6. After extinction of nicotine SA, the mPFC and VTA levels of the ionotropic glutamate receptor subunits, NR1, NR2A, NR2B, GluR1 and GluR2/3 were unaffected by prior nicotine SA. Following regional brain microdissection and punching, tissue extracts were prepared in SDS, separated by electrophoresis, and then subjected to western immunoblotting and quantitation, as described in the Methods. For all subunits, data were normalized to $\beta$-actin levels and then converted to mean percentage of saline $( \pm$ SEM) for each subunit. Representative blots comparing saline $(S)$ vs. nicotine (N)-treated animals are shown for each subunit. (Panel A) In mPFC, the previously reported enhancement of NMDA receptor subunit expression (e.g., NR2A and NR2B) by nicotine SA was no longer detectable (unpaired student $t$-test, $p>0.05$ for each subunit; $n=4$ per group) (Wang, Chen et al., 2007). (Panel B) Similarly, in the VTA, the reported elevation of the AMPA receptor subunit, GluR2/3, during nicotine SA, was not detected after extinction (unpaired student t-test, $\mathrm{p}>0.05$ for each subunit; $n=4$ per group). 
SA, there was no longer evidence of enhanced glutamate receptor subunit expression in mPFC or VTA, nor glutamate hyper-responsiveness to the intra-mPFC administration of NMDA.

\subsubsection{The effects of blockade of mPFC NMDA receptors on the maintenance of chronic nicotine SA and sucrose SA}

To evaluate the potential role of $\mathrm{mPFC}$ NMDA receptors in the maintenance of chronic nicotine $\mathrm{SA}$ in comparison to sucrose SA, in two separate groups of animals, the NMDA receptor antagonist, AP-5 (1.5 or $5 \mu \mathrm{g} /$ side), was microinjected into mPFC bilaterally 10 min before the initiation of an SA session. On d 19, 21 and 23, during both nicotine SA and sucrose SA, AP-5 or KRB was administered in a counterbalanced order. The intra-mPFC microinjection of AP-5 had no effect on active lever presses or the time required to obtain 100 sucrose pellets during chronic nicotine SA ( $>0.05$; Figure 3.7A) and sucrose SA ( $p>0.05$; Figure 3.7B), respectively. In a separate experiment, the pharmacological efficacy of the foregoing microinjection of AP- 5 was validated by microdialyzing the VTA following bilateral intra-mPFC NMDA \pm AP-5 microinjection. AP-5 (5 $\mu \mathrm{g} /$ side $)$ abolished the release of glutamate in the VTA $\left(\mathrm{F}_{1,3}=31.84, \mathrm{p}<0.05\right.$; data not shown). Therefore, NMDA receptors located in the $\mathrm{mPFC}$ are not required to maintain chronic nicotine or sucrose SA.

\subsubsection{The effects of blockade of VTA AMPA receptors on the maintenance of chronic nicotine SA and sucrose SA}

We have reported that glutamate receptor subunits (e.g., GluR2/3) up-regulate in the VTA during chronic nicotine SA (Wang et al., 2007). Therefore, experiments were designed to evaluate whether the AMPA receptor antagonist, NBQX, reduced chronic nicotine SA in comparison to sucrose SA, in two separate groups of animals; NBQX $(0.067$ or $0.25 \mu \mathrm{g} / \mathrm{side})$ or KRB was microinjected into VTA prior to SA sessions on d 19,21 and 23 in each paradigm. NBQX $(0.25 \mu \mathrm{g} / \mathrm{side})$ reduced the number of active lever presses during nicotine SA (Figure 3.8A) and increased the time required to obtain 100 sucrose pellets during sucrose $\mathrm{SA}$ (Figure $3.8 \mathrm{~B})\left(\mathrm{F}_{2,27}=8.89, \mathrm{p}<0.01\right.$ for chronic nicotine $\mathrm{SA} ; \mathrm{F}_{2,15}=35.59, \mathrm{p}<0.001$ for sucrose $\mathrm{SA}$ ). To determine whether the reduction in operant responding reflected an impairment of locomotion, we tested the effect of the intra-VTA administration NBQX $(0.25 \mu \mathrm{g} /$ side) on locomotion (Figure 3.8C). Pre-treatment with NBQX had no effect on locomotion $\left(\mathrm{F}_{2,15}=0.04, \mathrm{p}>0.05\right)$. Therefore, AMPA receptors in the VTA are required for nicotine reinforced and sucrose reinforced operant behaviors. 

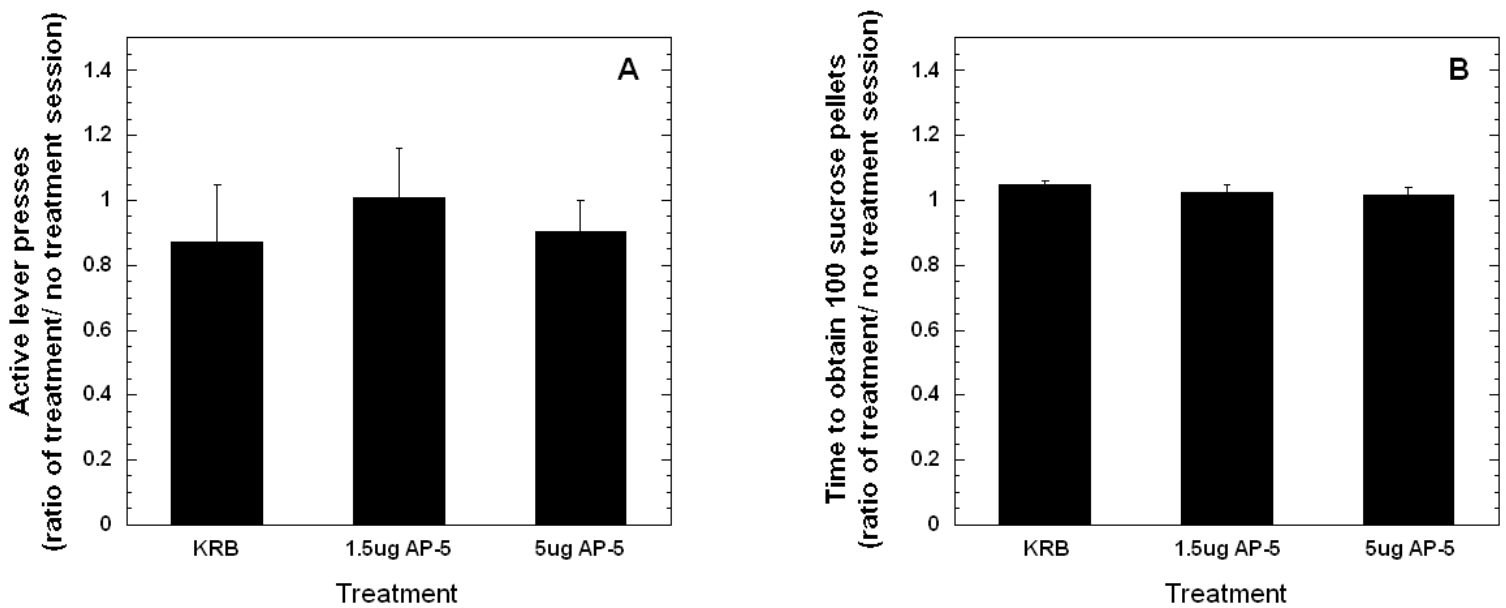

Figure 3.7. The NMDA receptor antagonist, AP-5, did not affect the maintenance of chronic nicotine or sucrose $\mathbf{S A}$. Using a counterbalanced design in two separate cohorts of animals (nicotine SA, panel A; sucrose SA, panel B), each animal received AP-5 (1.5 and $5 \mu \mathrm{g} /$ side) and KRB microinjected into $\mathrm{mPFC} 10 \mathrm{~min}$ before the initiation of daily test sessions on d 19, d21, and d 23, after $18 \mathrm{~d}$ of nicotine or sucrose SA. Animals selfadministered nicotine or sucrose on $\mathrm{d} 20$ and 22 without receiving $\mathrm{mPFC}$ injections. (Panel A) Active lever presses, expressed as the ratio of presses on the treatment day/presses on the preceding day without treatment, were not altered by AP-5 in the nicotine SA group (one way ANOVA: $F_{2,15}=0.25, p>0.05, n=6$ ). (Panel B) The time required to obtain 100 sucrose pellets in response to lever pressing (FR1) was expressed as the ratio of time required on the treatment day/time required on the preceding day without treatment. This ratio was not affected by intra-mPFC AP-5 (one way ANOVA, $\mathrm{F}_{2,25}=0.44, \mathrm{p}>0.05, \mathrm{n}=8-10$ per group). 

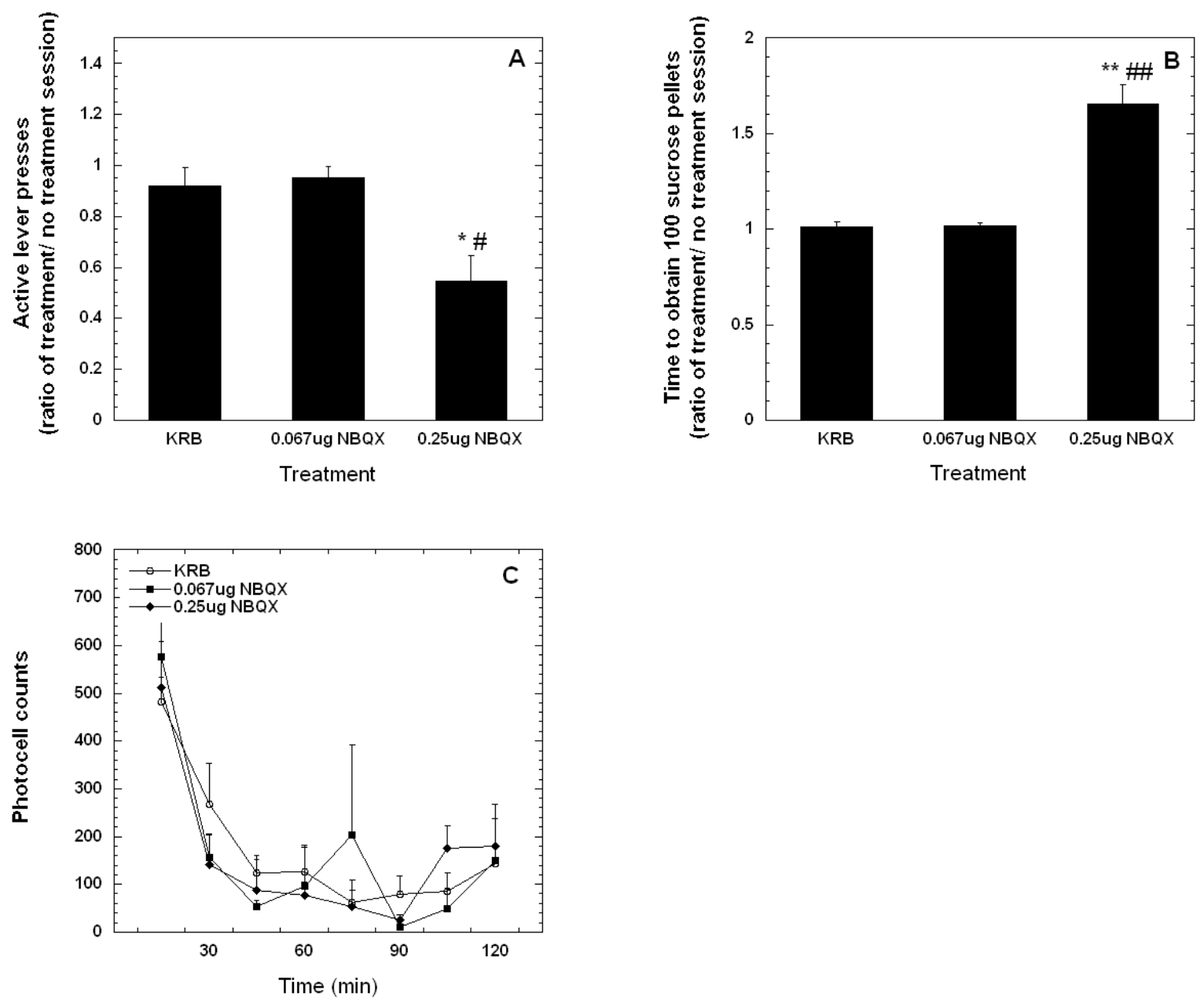

Figure 3.8. The AMPA receptor antagonist, NBQX, reduced active lever pressing during the maintenance phase of chronic nicotine and sucrose $\mathrm{SA}$ when administered into the VTA. Using a counterbalanced design in two separate cohorts of animals (nicotine SA, panel A; sucrose SA, panel B), each animal received NBQX (0.067 and $0.25 \mu \mathrm{g} /$ side) and KRB microinjected into VTA $10 \mathrm{~min}$ before the initiation of daily test sessions on d 19, d21, and d 23, after $18 \mathrm{~d}$ of nicotine or sucrose SA. Animals selfadministered nicotine or sucrose on d 20 and 22 without receiving VTA injections. (Panel A) Active lever presses, expressed as the ratio of presses on the treatment day/presses on the preceding day without treatment, were reduced by intra-VTA NBQX at $0.25 \mu \mathrm{g} / \mathrm{side}$ (one way ANOVA, $\mathrm{F}_{2,27}=8.90, \mathrm{p}<0.01, \mathrm{n}=10$ ). (Panel B) The time required to obtain 100 sucrose pellets in response to lever pressing (FR1) was expressed as the ratio of time required on the treatment day/time required on the preceding day without treatment. This ratio was increased by intra-VTA NBQX at $0.25 \mu \mathrm{g} / \mathrm{side}$ (one way ANOVA, $\mathrm{F}_{2,15}=35.60$, $\mathrm{p}<0.001, \mathrm{n}=6$ ). (Panel C) Intra-VTA NBQX did not affect locomotion (two way ANOVA with repeated measures, $\left.\mathrm{F}_{2,15}=0.04, \mathrm{p}>0.05\right)$. ${ }^{*}, \mathrm{p}<0.01,{ }^{* *}, \mathrm{p}<0.001$, compared to intramPFC or -VTA microinjection of KRB; \#, $\mathrm{p}<0.01$, \#\#, $\mathrm{p}<0.001$, compared to intra-mPFC or -VTA microinjection of NBQX $0.067 \mu \mathrm{g} /$ side. 


\subsection{Discussion}

Though many of the neurochemical and molecular effects of nicotine have been well studied, the drug's long-term neuroadaptive effects, specifically within the mesocortical glutamatergic pathway, are largely undefined. Based on recent evidence showing that NMDA and AMPA receptors are up-regulated in the mPFC and VTA, respectively, during chronic nicotine SA (Wang et al., 2007), we hypothesized that there may exist a hyperglutamatergic state of neurotransmission between the $\mathrm{mPFC}$ and VTA during chronic nicotine SA. The current study revealed enhanced glutamate responsiveness to stimulation with NMDA, not only at the site of perfusion within the $\mathrm{mPFC}$ but also from $\mathrm{mPFC}$ afferents to the VTA during the late maintenance phase of chronic nicotine SA (day 18). Enhanced glutamate responsiveness was only demonstrable during nicotine SA, and was undetectable after extinction. Furthermore, the previously reported up-regulation of ionotropic glutamate receptors did not persist after extinction. Behavioral studies indicated that the NMDA receptors in the $\mathrm{mPFC}$ were not required to maintain chronic nicotine SA, while VTA AMPA receptors were required. Blockade of AMPA receptors in the VTA decreased nicotine reinforced and sucrose reinforced operant behaviors without affecting the locomotion. Thus, neuroadaptive changes involving ionotropic glutamate receptors and NMDA receptor-dependent glutamate neurotransmission between $\mathrm{mPFC}$ and VTA are induced by nicotine SA. VTA AMPA receptors are necessary for the maintenance of nicotine SA.

The glutamatergic regulation of VTA dopamine neurons undergoes adaptive changes that are involved in the reinforcing effects of nicotine (Fagen et al., 2003). Nicotine stimulates dopamine secretion in the NAcc, an effect common to all drugs of abuse, in part by increasing glutamate release in the VTA, which in turn induces burst firing of dopamine neurons in the VTA (Grenhoff et al., 1986; Svensson et al., 1990; Mansvelder and McGehee, 2002). Nicotine induces long-term potentiation (LTP) of these dopamine neurons that depends on pre- and post-synaptic changes (Mansvelder and McGehee, 2000). The present study showed that $200 \mu \mathrm{M}$ NMDA, perfused into mPFC by microdialysis, significantly stimulated glutamate release in the $\mathrm{mPFC}$ and VTA of nicotine self-administering rats, but not controls. Although a concentration of $500 \mu \mathrm{M}$ NMDA induced glutamate release in the MPFC and VTA of both treatment groups, glutamate levels were significantly greater in the nicotine SA group. Thus, depending on the dose of NMDA, concurrent nicotine SA either potentiated or amplified glutamate release. In the VTA, these were not local effects of NMDA, indicating that nicotine SA had effected a state of enhanced glutamate neurotransmission.

Enhanced glutamate neurotransmission has been observed after extinction of cocaine and amphetamine. Ten d after 5 daily cocaine treatments, an injection of cocaine stimulated significantly more NAcc glutamate release in animals pretreated with the drug compared to saline (Reid and Berger, 1996). Similarly, intra-VTA AMPA stimulated more glutamate release in the VTA and NAcc of rats withdrawn from repeated daily injections of amphetamine than saline (Giorgetti et al., 2001). Thus, relatively few exposures to a psychostimulant are sufficient to induce a lasting state of enhanced glutamate responsiveness to the drug or to AMPA. Apparently, the amplification of 
glutamate responses to intra-VTA AMPA is indirect, mediated by the enduring effects of amphetamine on AMPA-stimulated dopamine and/or GABA neurotransmission in NAcc and VTA (Giorgetti et al., 2001).

The mechanism(s) whereby nicotine SA potentiates or amplifies NMDAstimulated glutamate secretion in $\mathrm{mPFC}$ and VTA are unknown, although the increased expression of NR2A and NR2B subunits and possibly NMDA receptors, expressed on pyramidal neurons in mPFC (Huntley et al., 1994), is likely to be involved. If the increase in $\mathrm{mPFC}$ NMDA receptor expression occurs on pyramidal neurons and is proportional to that reported previously for the subunits (Wang et al., 2007), the sensitivity to intramPFC NMDA would increase significantly; this would be likely to account for the amplification and possibly the potentiation of glutamate release during nicotine SA. Notwithstanding this, the potential role of GABA is worthy of consideration. GABA interneurons in mPFC inhibit pyramidal neurons (Tzschentke and Schmidt, 2000), and several reports have shown that GABA neurotransmission is affected by nicotine. Chronic injections of nicotine abolished the inhibitory effect of $\mathrm{GABA}_{\mathrm{B}}$ receptors on electrically evoked dopamine release in the VTA (Amantea and Bowery, 2004). In the $\mathrm{PFC}, \mathrm{GABA}_{\mathrm{B}}$ receptor $\mathrm{mRNA}$ was reduced by chronic exposure to nicotine, suggesting that nicotine SA might diminish the inhibitory regulation of pyramidal neurons (Li et al., 2004). If this occurs, one might expect this defect to spare GABA interneurons, since basal and NMDA-stimulated GABA release in $\mathrm{mPFC}$ was unaffected by nicotine SA in the present study. In contrast, a reduction in the expression of $\mathrm{GABA}_{\mathrm{B}}$ receptors on $\mathrm{PFC}$ GABA interneurons would probably be accompanied by increased basal GABA levels and enhanced NMDA-induced GABA release. Inhibitory regulation of $\mathrm{mPFC}$ pyramidal neurons has been demonstrated in experiments showing that stimulation of VTA glutamate release by intra-mPFC $\mathrm{KCl}$ was enhanced by blockade of $\mathrm{mPFC} \mathrm{GABA}_{\mathrm{B}}$ receptors (Harte and O'Connor, 2005). Thus, it is conceivable that both enhanced expression of NMDA receptors and reduced expression (or function) of GABA receptors on $\mathrm{mPFC}$ pyramidal neurons are responsible for the enhanced release of glutamate by NMDA.

After extinction from chronic nicotine SA, we no longer detected the augmentation of NMDA-induced glutamate release in mPFC or VTA. Furthermore, the increased expression of ionotropic glutamate receptor subunits in these regions had reverted to control levels. The reversal of adaptive changes in neurotransmission and receptor expression is consistent with findings from several psychostimulant studies showing that cellular and neurochemical adaptations are often transient. For example, decreased levels of VTA $\mathrm{G}_{\mathrm{i} \alpha}$ and $\mathrm{G}_{\mathrm{\alpha} \alpha}$ proteins, detectable at $1 \mathrm{~h}$ or $6 \mathrm{~h}$ after repeated injections of cocaine, were not present by $24 \mathrm{~h}$ (Nestler et al., 1990; Striplin and Kalivas, 1992). In a neurochemical study, AMPA-stimulated glutamate release in the VTA and NAcc was only augmented at $3 \mathrm{~d}$, but not $14 \mathrm{~d}$, after a regimen of repeated amphetamine treatments (Giorgetti et al., 2001). Finally, an electrophysiological study showed that the AMPA-induced firing of VTA dopamine neurons in vitro was enhanced at 3 but not $14 \mathrm{~d}$ following repeated exposure to cocaine or amphetamine (Zhang et al., 1997). The latter two studies indicate that the enhanced VTA glutamate neurotransmission following exposure to psychostimulants is transient and involves AMPA receptors, which were up- 
regulated in our previous study (Wang et al., 2007). Taken together, these studies all demonstrate that repeated exposure to psychostimulant drugs commonly induce molecular and neurochemical adaptations involving glutamate and dopamine neurotransmission in the mesolimbic system.

Since we found that glutamate receptor expression and glutamate neurotransmission were enhanced on d 19 of nicotine SA, studies were designed to determine whether NMDA and AMPA receptor antagonists impaired nicotine SA at this time. AP-5, an NMDA receptor antagonist, was microinjected bilaterally into the mPFC, where NMDA receptor subunit expression was increased (Wang et al., 2007); neither nicotine SA nor sucrose-reinforced operant behavior was affected. These observations are consistent with reports showing that blockade of NMDA receptors within the $\mathrm{MPFC}$ impaired the acquisition but not the expression of appetitive instrumental learning (Baldwin et al., 2000; Baldwin et al., 2002). Subsequently, NBQX, an AMPA receptor antagonist, was microinjected into the VTA, where GluR2/3 receptor subunit expression was increased (Wang et al., 2007); at the higher dose of NBQX, both nicotine SA and sucrose reinforced operant behavior was significantly inhibited. These observations suggest that VTA AMPA receptors are required for the maintenance of both operant behaviors. Thus, drug-induced neuroplasticity, involving the up-regulation of VTA GluR2/3-containing AMPA receptors, may not underlie the efficacy of NBQX. Thus, we hypothesize that VTA AMPA receptors may generally be required to maintain these appetitive operant behaviors.

AMPA receptors in the VTA are involved in behavioral, neurochemical and electrophysiological changes induced by chronic exposure to abused drugs. These receptors in the VTA are required for the initiation of behavioral sensitization to cocaine (Licata et al., 2004), and although the blockade of AMPA receptors by systemically administered antagonists failed to inhibit the expression of behavioral sensitization to these psychostimulants ( $\mathrm{Li}$ et al., 1997), such blockade was effective against ethanol sensitization (Broadbent et al., 2003). VTA AMPA receptors are also necessary for the initiation of morphine conditioned place preference (CPP) (Harris et al., 2004). Neurochemically, repeated exposure to amphetamine has been shown to augment the release of mesolimbic dopamine and glutamate by intra-VTA AMPA (Giorgetti et al., 2001). Electrophysiologically, AMPA receptors have been shown to stimulate and enhance burst firing of VTA dopamine neurons (Meltzer et al., 1997). Burst firing of dopamine neurons has been shown to be more efficient than single spike firing in the enhancement of phasic dopamine release and the transduction of salient stimuli (Chergui et al., 1993; Overton and Clark, 1997; Schultz et al., 1997). Nicotine increased the burst firing of VTA DA neurons (Grenhoff et al., 1986; Svensson et al., 1990) by stimulating glutamate release through presynaptic $\alpha 7$ receptors in VTA (Schilstrom et al., 2003). Additionally, a single injection of nicotine or other drugs of abuse (e.g. cocaine, damphetamine, ethanol) induced an increase in the strength of synapses on VTA dopamine neurons (i.e. increased AMPAR/NMDAR excitatory postsynaptic currents) (Saal et al., 2003). This is consistent with our report showing up-regulation of VTA GluR2/3, but not NMDA receptors, in the VTA during chronic nicotine SA (Wang et al., 2007). Taken together, these studies indicate that VTA AMPA receptors appear to manifest enhanced 
responsiveness and/or expression induced by various classes of abused drugs. These changes in function and expression of VTA AMPA receptors have been associated with enhanced dopamine and glutamate neurotransmission, and may be involved in behavioral sensitization and conditioned place preference to specific drugs of abuse. The current study indicates that VTA AMPA receptors are also involved in the maintenance of nicotine and food reinforced operant behavior.

These studies demonstrate the enhancement of glutamate neurotransmission in mPFC and VTA due to mPFC stimulation by NMDA during chronic nicotine SA. Based on recent neuroanatomical studies, the VTA glutamate measured herein may reflect two sources: direct glutamatergic projections from $\mathrm{mPFC}$ to VTA and glutamatergic fibers from LDTg and PPTg to VTA, activated by mPFC glutamatergic efferents to LDTg and PPTg (Tzschentke and Schmidt, 2000; Omelchenko and Sesack, 2005; Omelchenko and Sesack, 2007). This enhancement of glutamate neurotransmission is a transient adaptive change that correlates with the up-regulation of ionotropic glutamate receptors, requires coincident nicotine SA, and may specifically depend on the enhanced mPFC expression of NMDA receptors containing NR2A and NR2B subunits. Blockade of NMDA receptors in the $\mathrm{mPFC}$ did not affect the maintenance of chronic nicotine SA, while inhibition of VTA AMPA receptors decreased both nicotine and sucrose SA, indicating that VTA AMPA receptors may generally be required to maintain appetitive operant behavior. 


\section{Chapter 4. Discussion}

\subsection{The cellular distribution of up-regulated mPFC NMDA receptors and VTA AMPA receptors}

In the current study, we demonstrated that, after $18 \mathrm{~d}$ of nicotine SA, the expression of mPFC NR2A and NR2B receptor subunits was increased by $67 \%$ and $83 \%$, respectively, and the VTA GluR2/3 subunit was up-regulated by $34 \%$ (Wang et al., 2007). These nicotine-SA-induced increases in mPFC NR2A and $2 B$ subunits are likely to be incorporated into functional NMDA receptors, since previous studies indicate that the availability of NR2 subunits, but not the NR1 subunit, may be a limiting variable in the expression of intact NMDA receptors on the plasma membrane (see Chapter 2 Discussion). However, the functional significance of these up-regulated ionotropic glutamate receptors is largely unknown. Therefore, we examined the hypothesis that there may exist a hyperglutamatergic state of neurotransmission between $\mathrm{mPFC}$ and VTA. In rats self-administering nicotine for $18 \mathrm{~d}$, intra-mPFC perfusion of NMDA induced glutamate release in the $\mathrm{MPFC}$ and VTA that was potentiated or amplified compared to rats self-administering saline. Thus, it is plausible that up-regulation of ionotropic glutamate receptors within the $\mathrm{mPFC}$ and VTA may be one of the underlying mechanisms that contributes to the enhanced glutamate neurotransmission between these brain regions, depending upon their expression by specific types of neurons.

It has been reported that NMDA receptor subunits are differentially distributed in the brain at cellular, laminar (e.g. layer V of prefrontal cortex) and regional levels (Huntley et al., 1994). The expression pattern of NMDA receptors on a certain type(s) of neuron (e.g. glutamatergic pyramidal neuron or GABAergic interneuron) influences its functional effects on that neuron and on the microcircuitry within a particular brain region; this determines the effects of NMDA receptors on local neurotransmitter release (Huntley et al., 1994). Therefore, the up-regulated mPFC NMDA receptors, specifically the NR2A and NR2B subunits, might concentrate on a certain type of mPFC neuron, resulting in enhanced glutamate release in VTA when NMDA is administered into the mPFC. If the up-regulation of $\mathrm{mPFC}$ NMDA receptor expression occurs on pyramidal neurons and is proportional to that reported previously for the subunits (Wang et al., 2007), responsiveness to intra-mPFC NMDA would be expected to increase significantly; this most likely accounts for the observed amplification and possibly the potentiation of glutamate release during nicotine SA. In contrast, if the up-regulated NR2A and NR2B subunits were expressed by NMDA receptors located primarily on GABAergic interneurons in the $\mathrm{mPFC}$, then basal GABA levels would be expected to increase and glutamate secretion, induced by intra-mPFC NMDA would decrease during nicotine SA. However, we did not find an increase in basal or NMDA-stimulated GABA release. Thus, the up-regulated NR2A and NR2B subunits are most likely expressed by NMDA receptors located on $\mathrm{mPFC}$ pyramidal neurons.

In addition to the up-regulation of NMDA receptors, the GABA system may have a role in enhancing the $\mathrm{mPFC}$ glutamate response to NMDA during nicotine SA. GABA 
interneurons in mPFC inhibit pyramidal neurons (Tzschentke and Schmidt, 2000), and accumulating evidence has shown that GABA neutrotransmission is affected by nicotine (Amantea and Bowery, 2004; Li et al., 2004; Harte and O'Connor, 2005). For example, $\mathrm{mPFC} \mathrm{GABA}_{\mathrm{B}}$ receptors are known to be involved in the inhibitory regulation of $\mathrm{mPFC}$ pyramidal neurons, since intra-mPFC KCl-stimulated glutamate release in the VTA was enhanced by blockade of mPFC GABA $\mathrm{B}_{\mathrm{B}}$ receptors (Harte and O'Connor, 2005). In addition, after chronic nicotine administration, reduced sensitivity of $\mathrm{GABA}_{\mathrm{B}}$ receptors was found in the VTA (Amantea and Bowery, 2004), which may apply to the mPFC, as well. This reduced sensitivity of $\mathrm{GABA}_{\mathrm{B}}$ receptors may be due to reduced expression. Indeed, it has been shown that $\mathrm{mPFC} \mathrm{GABA}_{\mathrm{B}}$ receptor mRNA was reduced by chronic exposure to nicotine, indicating that nicotine $\mathrm{SA}$ might reduce the inhibitory regulation of pyramidal neurons, if the level of postsynaptic $\mathrm{GABA}_{\mathrm{B}}$ receptors decreased (Li et al., 2004). In our studies of nicotine $S A$, one would expect that presynaptic $G_{A B} A_{B}$ receptors on GABA interneurons were spared from such changes, since basal and NMDAstimulated GABA release in the $\mathrm{mPFC}$ were unaffected by nicotine $\mathrm{SA}$. Indeed, upon intra-mPFC infusion of a $\mathrm{GABA}_{\mathrm{B}}$ receptor agonist baclofen, glutamate levels in the $\mathrm{mPFC}$ were increased in the cocaine sensitized animals but not in the saline control animals, in contrast, GABA releases were reduced to similar extents, indicating the functions of postsynaptic and/or heterosynaptic $\mathrm{GABA}_{\mathrm{B}}$ receptors were impaired without affecting presynaptic $\mathrm{GABA}_{\mathrm{B}}$ autoreceptors after repeated cocaine exposure (Jayaram and Steketee, 2004) Thus, it is most likely that the increased expression of NMDA receptors and, perhaps, the reduced expression (or function) of $\mathrm{GABA}_{\mathrm{B}}$ receptors on $\mathrm{mPFC}$ pyramidal neurons, is responsible for the enhanced $\mathrm{mPFC}$ glutamate release induced by intra-mPFC NMDA.

In the VTA, AMPA receptors have been found on both GABA and DA neurons (Paquet et al., 1997). In the current study, we found that VTA GluR2/3 subunits of the AMPA receptor were up-regulated by $34 \%$ and VTA glutamate release was enhanced in rats self-administering nicotine for $18 \mathrm{~d}$. Anterograde and retrograde track-tracing studies done by Sesack et al., imply that mPFC glutamatergic neurons affect VTA GABA neurons through monosynaptic and polysynaptic connections to VTA (Carr and Sesack, 2000; Omelchenko and Sesack, 2005; Omelchenko and Sesack, 2007). Thus, changes in the activity of mPFC glutamatergic neurons will affect the excitability of VTA GABA neurons directly or indirectly (Tzschentke and Schmidt, 2000). VTA GABA release consists of somatodendritic and synaptic GABA from VTA GABA projection neurons and from GABA interneurons, respectively. Since we found that neither basal nor intramPFC NMDA-stimulated GABA secretion was affected by nicotine SA, it is unlikely that the up-regulated VTA GluR2/3 receptor subunit resides on the plasma membrane of GABA interneurons. This would most likely have increased basal and NMDA-stimulated VTA GABA release during nicotine SA, since GABA interneurons synapse with glutamatergic efferents from mPFC (Carr and Sesack, 2000). Our experiments do not address the potential for increased GluR2/3 expression by mesoaccumbens or mesocortical GABA neurons.

VTA dopamine neurons are affected by various drugs of abuse, including nicotine. A single injection of various drugs of abuse (e.g. cocaine, d-amphetamine, 
ethanol, nicotine) increased the strength of synapses on VTA dopamine neurons (i.e. increased AMPAR/NMDAR excitatory postsynaptic currents) (Saal et al., 2003), indicating that an alteration in the function or expression of synaptic AMPA and/or NMDA receptors on VTA dopamine neurons might be a common mechanism shared by several abused drugs. Although the underlying molecular mechanism(s) was not identified, enhanced AMPA receptor subunit expression upon chronic exposure to drugs of abuse appears to be a common mechanism (Fitzgerald et al., 1996; Wang et al., 2007).

AMPA receptor GluR2/3 subunits are expressed by a majority of VTA dopamine neurons. An immunocytochemical study has shown that GluR2/3 subunits were found on $63 \%$ of tyrosine hydroxylase positive neurons in the VTA. In comparison, GluR1 subunits were found on only $20 \%$ of these neurons (Chen et al., 2001). The up-regulated expression of GluR2/3 subunits in the VTA during chronic nicotine SA suggests there might be a larger pool of recycling of GluR2/3 subunits in VTA neurons, since these subunits are known to recycle constitutively which is important for the maintenance of basal synaptic activity (Passafaro et al., 2001; Shi, Hayashi et al., 2001). Thus, we postulate that the AMPAR/NMDAR EPSC ratio would be increased if more GluR2/3 subunits reside on the plasma membrane of VTA DA neurons during chronic nicotine SA.

\subsection{Monosynaptic and polysynaptic glutamatergic connections between MPFC and VTA}

The present study revealed enhanced glutamate neurotransmission between mPFC and VTA based on our observation of amplified or potentiated glutamate responsiveness in the VTA to stimulation by intra-mPFC NMDA. Recent evidence indicates that $\mathrm{mPFC}$ pyramidal neurons innervate VTA DA neurons via monosynaptic or polysynaptic connections (Carr and Sesack, 2000; Omelchenko and Sesack, 2005; Omelchenko and Sesack, 2007). Neuroanatomical tracing studies have demonstrated that mPFC pyramidal efferent neurons selectively innervate subpopulations of VTA dopamine and GABA neurons via monosynaptic connections. mPFC glutamatergic efferent pyramidal neurons synapse selectively on mesocortical but not mesoaccumbens DA neurons, whereas mesoaccumbens GABA neurons and not mesocortical GABA neurons are innervated by mPFC pyramidal neurons (Carr and Sesack, 2000).

The monosynaptic type of innervation between $\mathrm{mPFC}$ and VTA could not explain an in vivo microdialysis study showing that blockade of ionotropic glutamate receptors, using intra-VTA antagonists, decreased basal NAcc dopamine by $30 \%$ and blocked DA release evoked by mPFC stimulation (Taber et al., 1995). This study suggests that a polysynaptic glutamatergic pathway exists. Indeed, tract-tracing along with triple labeling revealed that the aforementioned DA release in the NAcc is most likely mediated by a polysynaptic glutamatergic pathway from $\mathrm{mPFC}$ that innervates the laterodorsal tegmental nucleus (LDTg) and pedunculopontine tegmental nucleus (PPTg), which in turn projects glutamatergic fibers to VTA mesoaccumbens DA neurons (Carr and Sesack, 2000; Omelchenko and Sesack, 2005). Furthermore, using vesicular glutamate transporter 
subtype 1 and 2 (VGlut1 and VGlut2), as markers for cortical and subcortical glutamate sources, respectively, it was demonstrated that VGlut2+ axon terminals were more highly expressed in VTA than VGlut1+ terminals (Omelchenko and Sesack, 2007). This suggests that the predominant source of glutamate to the VTA is from subcortical areas (Omelchenko and Sesack, 2007), possibly from LDTg and PPTg (Omelchenko and Sesack, 2005).

The polysynaptic connection between mPFC and VTA via LDTg and/or PPTg might play a major role in intra-mPFC NMDA-stimulated glutamate release in VTA during chronic nicotine SA. A study combining anterograde and retrograde tract-tracing of LDTg projections to VTA neurons found that LDTg glutamatergic projections preferentially excite mesoaccumbens DA neurons (Omelchenko and Sesack, 2005). Thus, the polysynaptic connection between mPFC and VTA, specifically via LDTg, may be important for regulating VTA mesoaccumbens DA neuron activity. However, further experiments are needed to clarify the role of LDTg and/or PPTg in the amplification of NMDA-induced glutamate neurotransmission between $\mathrm{mPFC}$ and VTA during chronic nicotine SA. For example, a study that blocked ionotropic glutamate receptors in the LDTg and/or PPTg and stimulated with intra-mPFC NMDA during chronic nicotine SA would indicate to what extent LDTg and/or PPTg are involved.

In summary, the activation of both polysynaptic and monosynaptic glutamatergic $\mathrm{mPFC}$ efferents appears to regulate VTA glutamate levels. The polysynaptic efferents, possibly via LDTg and or PPTg, may be more important in regulating VTA mesoaccumbens DA neuron activity. Such efferents may also be the major sources of the enhanced VTA glutamate, although further experiments are required to test this hypothesis.

\subsection{The potential functional significance of enhanced glutamate neurotransmission during chronic nicotine SA}

The reinforcing effects of nicotine, the major psychoactive component in cigarette smoke, depend on dopamine secretion in the mesolimbic system of the brain (Corrigall and Coen, 1989; Corrigall et al., 1992). Recent reports have shown that nicotine acutely stimulates VTA glutamate release through presynaptic nAChRs, possibly $\alpha 7$ subunits, which in turn excites VTA DA neurons (Fu et al., 2000; Mansvelder and McGehee, 2000). It has been shown that dopamine release in the NAcc at least partly depends on VTA ionotropic glutamate receptors. For example, nicotine-stimulated NAcc DA release is reduced after intra-VTA infusion of the NMDA receptor antagonists (AP-5 or CGS19755), suggesting NMDA receptors in the VTA are required (Schilstrom et al., 1998; Fu et al., 2000). VTA DA neurons receive glutamatergic innervation from mPFC and subcortical areas. Thus, it is conceivable that nicotine also may increase the activity of VTA dopamine neurons by activating glutamatergic inputs from these areas, which might be a mechanism in addition to its direct stimulating effect. Indeed, the glutamatedependent burst firing of VTA DA neurons is well documented (see discussion below). 
Consistent with this, in the present study, we found that NMDA-stimulated glutamate neurotransmission within $\mathrm{MPFC}$ and VTA was enhanced during chronic nicotine SA.

VTA dopamine neurons exhibit a "tonic, irregular activity pattern" (Omelchenko and Sesack, 2005), consisting of single spikes and bursts of action potentials (Overton and Clark, 1997), from recordings of naïve animals in vivo and in vitro (Sanghera et al., 1984; Grace and Onn, 1989). Compared with single spike activity, burst firing activity is more efficient at transducing salient environmental stimuli (Overton and Clark, 1997; Schultz et al., 1997; Berridge and Robinson, 1998) and enhancing phasic DA release (Chergui et al., 1993). The fluctuation of phasic DA levels in the mPFC and NAcc may signal future expectancy (Schultz et al., 1997; Omelchenko and Sesack, 2005), or attentional shifting (Redgrave et al., 1999). Nicotine has been shown to increase the firing rate and burst firing of VTA DA neurons (Nisell et al., 1996; Fisher et al., 1998), contributing to phasic DA release. However, in studies of VTA slice preparations, nicotine did not induce burst firing (Calabresi et al., 1989), suggesting that nicotinestimulated glutamate release from cortical and subcortical areas is critical for the effect of nicotine on VTA DA neuronal burst firing activity in vivo. Indeed, burst firing of VTA DA neurons is critically dependent on mPFC glutamate inputs (Svensson et al., 1990). Chemical (Murase et al., 1993) or electrical (Gariano and Groves, 1988; Tong et al., 1996) stimulation of mPFC increases burst firing of VTA DA neurons.

In line with these observations, in the present study, chronic nicotine SA induced enhanced glutamate release in the $\mathrm{mPFC}$ and VTA in response to intra-mPFC NMDA. This increase in VTA glutamate release may stimulate more burst firing by VTA DA neurons due to enhanced NMDA receptor sensitivity. Indeed, the spontaneous burst firing of VTA DA neurons are largely determined by the level of activation of NMDA receptors on these neurons (Schilstrom et al., 2003). Activation of the NMDA receptor has been shown to be important for the induction of burst firing activity in the VTA; nicotine does not induce burst firing in VTA DA neurons in vitro. In contrast, NMDA is still capable of doing so (Johnson et al., 1992). However, NMDA receptors can only be activated when neurons are depolarized to remove the $\mathrm{Mg}^{2+}$ blockade. Thus, the enhanced intra-mPFC NMDA-stimulated glutamate neurotransmission in the VTA may increase the responsiveness of NMDA receptors on VTA DA neurons by increasing the probability of depolarization, which would remove the $\mathrm{Mg}^{2+}$ blockade. If the up-regulated GluR2/3 subunit resides on plasma membrane of VTA DA neurons, the synaptic strength (i.e. the AMPAR/NMDAR EPSC) also could be enhanced, which may further contribute to burst firing of VTA DA neurons during chronic nicotine SA.

\subsection{Enhanced glutamate neurotransmission implicated in behavioral paradigms affected by chronic administration of drugs of abuse}

Behavioral sensitization refers to an enhanced motor-stimulant response to most abused drugs including cocaine, amphetamine and nicotine, after repeated or intermittent exposure (Steketee, 2005). Evidence indicates that blockade of ionotropic glutamate receptors prevents initiation or expression of behavioral sensitization to different drugs of 
abuse (see Chapter 3 Discussion). In addition, it has been shown that repeated exposure to psychostimulants (e.g. cocaine or amphetamine) induces enhanced glutamate responsiveness within the mesocorticolimbic system to drugs or to ionotropic glutamate receptor agonists that were administered under the same treatment paradigms that induced behavioral sensitization. For example, enhanced glutamate neurotransmission has been observed after cessation of cocaine and amphetamine injections. Ten $\mathrm{d}$ after 5 daily cocaine treatments, an injection of cocaine stimulated significantly more NAcc glutamate release in animals pretreated with the drug compared to saline (Reid and Berger, 1996). Similarly, intra-VTA AMPA stimulated more glutamate release in the VTA and NAcc of rats withdrawn from repeated daily injections of amphetamine than saline (Giorgetti et al., 2001). Functionally, this enhanced glutamate neurotransmission might contribute to increased VTA DA neuron responsiveness to glutamate, as measured by a current-response curve (White et al., 1995) which specifically involves AMPA receptors (Zhang et al., 1997). Additionally, enhanced VTA AMPA receptor expression after repeated exposure of drugs of abuse has been documented extensively (see Chapter 2 Discussion). NAcc surface AMPA receptor expression was increased $21 \mathrm{~d}$ after the last injection in cocaine-sensitized animals, but not in rats that did not develop behavioral sensitization. This suggests that up-regulation of NAcc AMPA receptor surface expression might contribute to behavioral sensitization (Boudreau and Wolf, 2005). In line with these evidences, we found a $34 \%$ increase in the GluR2/3 subunit and enhanced glutamate neurotransmission in the VTA. The precise functional significance of this finding is unknown, however, we demonstrated that VTA AMPA receptors were required for the maintenance of both chronic nicotine SA and sucrose SA operant behaviors, suggesting VTA AMPA receptors may be generally required for the maintenance of these appetitive operant behaviors.

In drug abuse research, reinstatement models, characterized by the cue-, stress- or drug priming-induced reinstatement of unreinforced lever pressing behavior that was previously extinguished, are used to understand relapse to drug seeking behavior in human addicts (Shaham et al., 2003). Enhanced glutamate neurotransmission within the pathway from $\mathrm{mPFC}$ to NAcc core has been shown to be the key mediator of various forms of relapse to cocaine seeking, such as cocaine-priming induced (McFarland et al., 2003) and footshock-induced cocaine seeking behavior (McFarland et al., 2004). In cocaine priming-induced reinstatement, increased glutamate release in the NAcc core after a cocaine-priming injection was only found in cocaine SA animals, and not in the yoked saline or yoked cocaine group. Moreover, the enhanced glutamate release in the NAcc core was not associated with food-induced food seeking behavior nor lever responding per se. This indicates a positive and specific association between enhanced glutamate neurotransmission and cocaine-induced reinstatement of cocaine seeking behavior (McFarland et al., 2003). In addition, enhanced DA release in NAcc core was found in all groups (e.g. yoked saline, yoked cocaine, cocaine SA) after the cocainepriming injection, suggesting that enhanced DA release might be a secondary effect of reinstatement of cocaine seeking behavior, rather than the primary cause (McFarland et al., 2003). Furthermore, when dorsal PFC was inactivated by microinfusion of the $\mathrm{GABA}_{\mathrm{B}}$ agonist, baclofen, and the $\mathrm{GABA}_{\mathrm{A}}$ agonist, muscimol, the enhanced glutamate release in the NAcc core and cocaine-induced reinstatement of cocaine seeking behavior 
were abolished concurrently, suggesting that the source of the elevated glutamate secretion in the NAcc core after cocaine challenge was the dorsal PFC (activity dependent) (McFarland et al., 2003). Similarly, footshock-induced cocaine seeking behavior was also associated with increased glutamate release in the NAcc core via dorsal PFC pwrojections (McFarland et al., 2004). Overall, these observations suggest that enhanced glutamate neurotransmission between $\mathrm{mPFC}$ and NAcc core underlies cocaine-priming and footshock-induced cocaine reinstatement.

In current study, we hypothesized that mPFC NMDA or VTA AMPA receptors, which up-regulated during chronic nicotine SA, might be required to maintain operant responding behavior during late maintenance phase of chronic nicotine SA. Data suggested that $\mathrm{mPFC}$ NMDA receptors might not be required to maintain nicotine or sucrose SA, which is consistent with the notion that blockade of NMDA receptors within the $\mathrm{mPFC}$ impaired the acquisition but not the expression of appetitive instrumental learning (Baldwin et al., 2000; Baldwin et al., 2002). In contrast, NBQX, an AMPA receptor antagonist, was microinjected into the VTA, where GluR2/3 receptor subunit expression was increased (Wang et al., 2007); at the higher dose of NBQX, both nicotine $\mathrm{SA}$ and sucrose reinforced operant behavior was significantly inhibited. Moreover, this inhibitory effect of NBQX on operant behavior was not due to an inhibitory effect on locomotion. These observations suggest that VTA AMPA receptors are required for the maintenance of both operant behaviors.

\subsection{Conclusion}

In current study, we demonstrated that, after $18 \mathrm{~d}$ chronic nicotine SA, iGluRs upregulated in the mesocortical regions, specifically, in the $\mathrm{mPFC}$ and VTA. The glutamate but not GABA release stimulated by intra-mPFC NMDA in the MPFC and VTA was potentiated or augmented in rats self-administered nicotine compared with rats selfadministered saline, indicating a hyperglutamatergic neurotransmission between $\mathrm{mPFC}$ and VTA. These neurochemical changes appeared to be transient: after $10 \mathrm{~d}$ of extinction, the enhanced glutamate neurotransmission and up-regulated iGluRs were no longer demonstrable. In addition, blockade of $\mathrm{mPFC}$ NMDA receptors did not affect the maintenance of chronic nicotine SA and sucrose SA. In contrast, blockade of VTA AMPA receptors inhibited the maintenance of both SA behaviors, suggesting VTA AMPA receptors may generally be required to maintain appetitive operant behavior. In summary, the current study demonstrated a transient neuroadaptive change in the mesocortical glutamate system during chronic nicotine SA, and such changes might contribute to the augmented mesocorticolimbic dopamine neurotransmission which is common to all drugs of abuse. 


\section{List of References}

Adell, A. and Artigas, F. (2004) The somatodendritic release of dopamine in the ventral tegmental area and its regulation by afferent transmitter systems. Neurosci

Biobehav Rev, 28, 415-31.

Alkondon, M. and Albuquerque, E. X. (1993) Diversity of nicotinic acetylcholine receptors in rat hippocampal neurons. I. Pharmacological and functional evidence for distinct structural subtypes. J Pharmacol Exp Ther, 265, 1455-73.

Alkondon, M., Pereira, E. F. and Albuquerque, E. X. (1998) alpha-bungarotoxin- and methyllycaconitine-sensitive nicotinic receptors mediate fast synaptic transmission in interneurons of rat hippocampal slices. Brain Res, 810, 257-63.

Alkondon, M., Pereira, E. F., Eisenberg, H. M. and Albuquerque, E. X. (1999) Choline and selective antagonists identify two subtypes of nicotinic acetylcholine receptors that modulate GABA release from CA1 interneurons in rat hippocampal slices. J Neurosci, 19, 2693-705.

Amantea, D. and Bowery, N. G. (2004) Reduced inhibitory action of a GABAB receptor agonist on $[3 \mathrm{H}]$-dopamine release from rat ventral tegmental area in vitro after chronic nicotine administration. BMC Pharmacol, 4, 24.

Andreoli, M., Tessari, M., Pilla, M., Valerio, E., Hagan, J. J. and Heidbreder, C. A. (2003) Selective antagonism at dopamine D3 receptors prevents nicotinetriggered relapse to nicotine-seeking behavior. Neuropsychopharmacology, 28, 1272-80.

Backstrom, P. and Hyytia, P. (2007) Involvement of AMPA/kainate, NMDA, and mGlu5 receptors in the nucleus accumbens core in cue-induced reinstatement of cocaine seeking in rats. Psychopharmacology (Berl).

Bacon, S. J., Headlam, A. J., Gabbott, P. L. and Smith, A. D. (1996) Amygdala input to medial prefrontal cortex (mPFC) in the rat: a light and electron microscope study. Brain Res, 720, 211-9.

Baldwin, A. E., Holahan, M. R., Sadeghian, K. and Kelley, A. E. (2000) N-methyl-Daspartate receptor-dependent plasticity within a distributed corticostriatal network mediates appetitive instrumental learning. Behav Neurosci, 114, 84-98.

Baldwin, A. E., Sadeghian, K. and Kelley, A. E. (2002) Appetitive instrumental learning requires coincident activation of NMDA and dopamine D1 receptors within the medial prefrontal cortex. J Neurosci, 22, 1063-71. 
Barria, A. and Malinow, R. (2002) Subunit-specific NMDA receptor trafficking to synapses. Neuron, 35, 345-53.

Berendse, H. W., Galis-de Graaf, Y. and Groenewegen, H. J. (1992) Topographical organization and relationship with ventral striatal compartments of prefrontal corticostriatal projections in the rat. J Comp Neurol, 316, 314-47.

Berridge, K. C. and Robinson, T. E. (1998) What is the role of dopamine in reward: hedonic impact, reward learning, or incentive salience? Brain Res Brain Res Rev, 28, 309-69.

Blokhina, E. A., Kashkin, V. A., Zvartau, E. E., Danysz, W. and Bespalov, A. Y. (2005) Effects of nicotinic and NMDA receptor channel blockers on intravenous cocaine and nicotine self-administration in mice. Eur Neuropsychopharmacol, 15, 219-25.

Boudreau, A. C. and Wolf, M. E. (2005) Behavioral sensitization to cocaine is associated with increased AMPA receptor surface expression in the nucleus accumbens. $J$ Neurosci, 25, 9144-51.

Broadbent, J., Kampmueller, K. M. and Koonse, S. A. (2003) Expression of behavioral sensitization to ethanol by DBA/2J mice: the role of NMDA and non-NMDA glutamate receptors. Psychopharmacology (Berl), 167, 225-34.

Broide, R. S. and Leslie, F. M. (1999) The alpha7 nicotinic acetylcholine receptor in neuronal plasticity. Mol Neurobiol, 20, 1-16.

Brower, V. G., Fu, Y., Matta, S. G. and Sharp, B. M. (2002) Rat strain differences in nicotine self-administration using an unlimited access paradigm. Brain Res, 930, $12-20$.

Brown, R. W. and Kolb, B. (2001) Nicotine sensitization increases dendritic length and spine density in the nucleus accumbens and cingulate cortex. Brain Res, 899, 94100 .

Buczek, Y., Le, A. D., Wang, A., Stewart, J. and Shaham, Y. (1999) Stress reinstates nicotine seeking but not sucrose solution seeking in rats. Psychopharmacology (Berl), 144, 183-8.

Buisson, B. and Bertrand, D. (2001) Chronic exposure to nicotine upregulates the human (alpha)4((beta)2 nicotinic acetylcholine receptor function. J Neurosci, 21, 181929.

Buisson, B. and Bertrand, D. (2002) Nicotine addiction: the possible role of functional upregulation. Trends Pharmacol Sci, 23, 130-6. 
Calabresi, P., Lacey, M. G. and North, R. A. (1989) Nicotinic excitation of rat ventral tegmental neurones in vitro studied by intracellular recording. Br J Pharmacol, 98, $135-40$.

Capriles, N., Rodaros, D., Sorge, R. E. and Stewart, J. (2003) A role for the prefrontal cortex in stress- and cocaine-induced reinstatement of cocaine seeking in rats. Psychopharmacology (Berl), 168, 66-74.

Carlezon, W. A., Jr. and Nestler, E. J. (2002) Elevated levels of GluR1 in the midbrain: a trigger for sensitization to drugs of abuse? Trends Neurosci, 25, 610-5.

Carr, D. B. and Sesack, S. R. (2000) GABA-containing neurons in the rat ventral tegmental area project to the prefrontal cortex. Synapse, 38, 114-23.

Carr, D. B. and Sesack, S. R. (2000) Projections from the rat prefrontal cortex to the ventral tegmental area: target specificity in the synaptic associations with mesoaccumbens and mesocortical neurons. J Neurosci, 20, 3864-73.

Cartier, G. E., Yoshikami, D., Gray, W. R., Luo, S., Olivera, B. M. and McIntosh, J. M. (1996) A new alpha-conotoxin which targets alpha3beta2 nicotinic acetylcholine receptors. J Biol Chem, 271, 7522-8.

Cartmell, J. and Schoepp, D. D. (2000) Regulation of neurotransmitter release by metabotropic glutamate receptors. J Neurochem, 75, 889-907.

Centers for Disease Control. (2002) Annual smoking-attributable mortality, years of potential life lost, and economic costs--United States, 1995-1999. MMWR Morb Mortal Wkly Rep, 51, 300-3.

Centers for Disease Control. (2005) Annual smoking-attributable mortality, years of potential life lost, and productivity losses--United States, 1997-2001. MMWR Morb Mortal Wkly Rep, 54, 625-8.

Chandler, L. J., Norwood, D. and Sutton, G. (1999) Chronic ethanol upregulates NMDA and AMPA, but not kainate receptor subunit proteins in rat primary cortical cultures. Alcohol Clin Exp Res, 23, 363-70.

Chen, L. W., Wei, L. C., Lang, B., Ju, G. and Chan, Y. S. (2001) Differential expression of AMPA receptor subunits in dopamine neurons of the rat brain: a double immunocytochemical study. Neuroscience, 106, 149-60.

Chergui, K., Charlety, P. J., Akaoka, H., Saunier, C. F., Brunet, J. L., Buda, M., Svensson, T. H. and Chouvet, G. (1993) Tonic activation of NMDA receptors causes spontaneous burst discharge of rat midbrain dopamine neurons in vivo. Eur J Neurosci, 5, 137-44. 
Churchill, L., Swanson, C. J., Urbina, M. and Kalivas, P. W. (1999) Repeated cocaine alters glutamate receptor subunit levels in the nucleus accumbens and ventral tegmental area of rats that develop behavioral sensitization. J Neurochem, $\mathbf{7 2}$, 2397-403.

Conde, F., Maire-Lepoivre, E., Audinat, E. and Crepel, F. (1995) Afferent connections of the medial frontal cortex of the rat. II. Cortical and subcortical afferents. J Comp Neurol, 352, 567-93.

Corrigall, W. A. (1999) Nicotine self-administration in animals as a dependence model. Nicotine Tob Res, 1, 11-20.

Corrigall, W. A. and Coen, K. M. (1989) Nicotine maintains robust self-administration in rats on a limited-access schedule. Psychopharmacology (Berl), 99, 473-8.

Corrigall, W. A. and Coen, K. M. (1991) Opiate antagonists reduce cocaine but not nicotine self-administration. Psychopharmacology (Berl), 104, 167-70.

Corrigall, W. A. and Coen, K. M. (1991) Selective dopamine antagonists reduce nicotine self-administration. Psychopharmacology (Berl), 104, 171-6.

Corrigall, W. A. and Coen, K. M. (1994) Nicotine self-administration and locomotor activity are not modified by the 5-HT3 antagonists ICS 205-930 and MDL 72222. Pharmacol Biochem Behav, 49, 67-71.

Corrigall, W. A., Coen, K. M., Adamson, K. L., Chow, B. L. and Zhang, J. (2000) Response of nicotine self-administration in the rat to manipulations of mu-opioid and gamma-aminobutyric acid receptors in the ventral tegmental area.

Psychopharmacology (Berl), 149, 107-14.

Corrigall, W. A., Coen, K. M., Zhang, J. and Adamson, K. L. (2001) GABA mechanisms in the pedunculopontine tegmental nucleus influence particular aspects of nicotine self-administration selectively in the rat. Psychopharmacology (Berl), 158, 190-7.

Corrigall, W. A., Coen, K. M., Zhang, J. and Adamson, L. (2002) Pharmacological manipulations of the pedunculopontine tegmental nucleus in the rat reduce selfadministration of both nicotine and cocaine. Psychopharmacology (Berl), 160, 198-205.

Corrigall, W. A., Franklin, K. B., Coen, K. M. and Clarke, P. B. (1992) The mesolimbic dopaminergic system is implicated in the reinforcing effects of nicotine. Psychopharmacology (Berl), 107, 285-9.

Cox, B. M., Goldstein, A. and Nelson, W. T. (1984) Nicotine self-administration in rats. Br J Pharmacol, 83, 49-55. 
Dani, J. A. and Bertrand, D. (2007) Nicotinic Acetylcholine Receptors and Nicotinic Cholinergic Mechanisms of the Central Nervous System. Annu Rev Pharmacol Toxicol, 47, 699-729.

Dani, J. A. and De Biasi, M. (2001) Cellular mechanisms of nicotine addiction. Pharmacol Biochem Behav, 70, 439-46.

Dani, J. A., Ji, D. and Zhou, F. M. (2001) Synaptic plasticity and nicotine addiction. Neuron, 31, 349-52.

Davenport, K. E., Houdi, A. A. and Van Loon, G. R. (1990) Nicotine protects against mu-opioid receptor antagonism by beta-funaltrexamine: evidence for nicotineinduced release of endogenous opioids in brain. Neurosci Lett, 113, 40-6.

Dewey, S. L., Brodie, J. D., Gerasimov, M., Horan, B., Gardner, E. L. and Ashby, C. R., Jr. (1999) A pharmacologic strategy for the treatment of nicotine addiction. Synapse, 31, 76-86.

Di Chiara, G. (2000) Role of dopamine in the behavioural actions of nicotine related to addiction. Eur J Pharmacol, 393, 295-314.

Di Chiara, G. and Imperato, A. (1988) Drugs abused by humans preferentially increase synaptic dopamine concentrations in the mesolimbic system of freely moving rats. Proc Natl Acad Sci U S A, 85, 5274-8.

Erb, S., Hitchcott, P. K., Rajabi, H., Mueller, D., Shaham, Y. and Stewart, J. (2000) Alpha-2 adrenergic receptor agonists block stress-induced reinstatement of cocaine seeking. Neuropsychopharmacology, 23, 138-50.

Fagen, Z. M., Mansvelder, H. D., Keath, J. R. and McGehee, D. S. (2003) Short- and long-term modulation of synaptic inputs to brain reward areas by nicotine. Ann $N$ Y Acad Sci, 1003, 185-95.

Fattore, L., Cossu, G., Martellotta, M. C. and Fratta, W. (2002) Baclofen antagonizes intravenous self-administration of nicotine in mice and rats. Alcohol Alcohol, 37, 495-8.

Ferrari, R., Le Novere, N., Picciotto, M. R., Changeux, J. P. and Zoli, M. (2002) Acute and long-term changes in the mesolimbic dopamine pathway after systemic or local single nicotine injections. Eur J Neurosci, 15, 1810-8.

Fisher, J. L., Pidoplichko, V. I. and Dani, J. A. (1998) Nicotine modifies the activity of ventral tegmental area dopaminergic neurons and hippocampal GABAergic neurons. J Physiol Paris, 92, 209-13. 
Fitzgerald, L. W., Ortiz, J., Hamedani, A. G. and Nestler, E. J. (1996) Drugs of abuse and stress increase the expression of GluR1 and NMDAR1 glutamate receptor subunits in the rat ventral tegmental area: common adaptations among crosssensitizing agents. J Neurosci, 16, 274-82.

Forster, G. L. and Blaha, C. D. (2000) Laterodorsal tegmental stimulation elicits dopamine efflux in the rat nucleus accumbens by activation of acetylcholine and glutamate receptors in the ventral tegmental area. Eur J Neurosci, 12, 3596-604.

Frazier, C. J., Buhler, A. V., Weiner, J. L. and Dunwiddie, T. V. (1998) Synaptic potentials mediated via alpha-bungarotoxin-sensitive nicotinic acetylcholine receptors in rat hippocampal interneurons. $J$ Neurosci, 18, 8228-35.

Fu, Y., Matta, S. G., Brower, V. G. and Sharp, B. M. (2001) Norepinephrine secretion in the hypothalamic paraventricular nucleus of rats during unlimited access to selfadministered nicotine: An in vivo microdialysis study. J Neurosci, 21, 8979-89.

Fu, Y., Matta, S. G., Gao, W., Brower, V. G. and Sharp, B. M. (2000) Systemic nicotine stimulates dopamine release in nucleus accumbens: re-evaluation of the role of $\mathrm{N}$ methyl-D-aspartate receptors in the ventral tegmental area. J Pharmacol Exp Ther, 294, 458-65.

Fu, Y., Matta, S. G., Kane, V. B. and Sharp, B. M. (2003) Norepinephrine release in amygdala of rats during chronic nicotine self-administration: an in vivo microdialysis study. Neuropharmacology, 45, 514-23.

Fu, Y., Matta, S. G., Valentine, J. D. and Sharp, B. M. (1997) Adrenocorticotropin response and nicotine-induced norepinephrine secretion in the rat paraventricular nucleus are mediated through brainstem receptors. Endocrinology, 138, 1935-43.

Gabbott, P. L., Warner, T. A., Jays, P. R., Salway, P. and Busby, S. J. (2005) Prefrontal cortex in the rat: projections to subcortical autonomic, motor, and limbic centers. J Comp Neurol, 492, 145-77.

Gariano, R. F. and Groves, P. M. (1988) Burst firing induced in midbrain dopamine neurons by stimulation of the medial prefrontal and anterior cingulate cortices. Brain Res, 462, 194-8.

Giorgetti, M., Hotsenpiller, G., Ward, P., Teppen, T. and Wolf, M. E. (2001) Amphetamine-induced plasticity of AMPA receptors in the ventral tegmental area: effects on extracellular levels of dopamine and glutamate in freely moving rats. $J$ Neurosci, 21, 6362-9.

Giovino, G. A., Henningfield, J. E., Tomar, S. L., Escobedo, L. G. and Slade, J. (1995) Epidemiology of tobacco use and dependence. Epidemiol Rev, 17, 48-65. 
Goldberg, S. R., Spealman, R. D. and Goldberg, D. M. (1981) Persistent behavior at high rates maintained by intravenous self-administration of nicotine. Science, 214, 5735.

Gonzalez, C. L., Gharbawie, O. A., Whishaw, I. Q. and Kolb, B. (2005) Nicotine stimulates dendritic arborization in motor cortex and improves concurrent motor skill but impairs subsequent motor learning. Synapse, 55, 183-91.

Gotti, C., Carbonnelle, E., Moretti, M., Zwart, R. and Clementi, F. (2000) Drugs selective for nicotinic receptor subtypes: a real possibility or a dream? Behav Brain Res, 113, 183-92.

Grace, A. A. and Onn, S. P. (1989) Morphology and electrophysiological properties of immunocytochemically identified rat dopamine neurons recorded in vitro. $J$ Neurosci, 9, 3463-81.

Grenhoff, J., Aston-Jones, G. and Svensson, T. H. (1986) Nicotinic effects on the firing pattern of midbrain dopamine neurons. Acta Physiol Scand, 128, 351-8.

Groenewegen, H. J., Berendse, H. W., Wolters, J. G. and Lohman, A. H. (1990) The anatomical relationship of the prefrontal cortex with the striatopallidal system, the thalamus and the amygdala: evidence for a parallel organization. Prog Brain Res, 85, 95-116; discussion 116-8.

Grottick, A. J., Corrigall, W. A. and Higgins, G. A. (2001) Activation of 5-HT(2C) receptors reduces the locomotor and rewarding effects of nicotine. Psychopharmacology (Berl), 157, 292-8.

Guo, J. Z., Tredway, T. L. and Chiappinelli, V. A. (1998) Glutamate and GABA release are enhanced by different subtypes of presynaptic nicotinic receptors in the lateral geniculate nucleus. J Neurosci, 18, 1963-9.

Hall, R. A. and Soderling, T. R. (1997) Differential surface expression and phosphorylation of the N-methyl-D-aspartate receptor subunits NR1 and NR2 in cultured hippocampal neurons. J Biol Chem, 272, 4135-40.

Hanson, H. M., Ivester, C. A. and Morton, B. R. (1979) Nicotine self-administration in rats. NIDA Res Monogr, 70-90.

Harris, G. C. and Aston-Jones, G. (2003) Critical role for ventral tegmental glutamate in preference for a cocaine-conditioned environment. Neuropsychopharmacology, 28, 73-6.

Harris, G. C., Wimmer, M., Byrne, R. and Aston-Jones, G. (2004) Glutamate-associated plasticity in the ventral tegmental area is necessary for conditioning environmental stimuli with morphine. Neuroscience, 129, 841-7. 
Harte, M. and O'Connor, W. T. (2004) Evidence for a differential medial prefrontal dopamine D1 and D2 receptor regulation of local and ventral tegmental glutamate and GABA release: a dual probe microdialysis study in the awake rat. Brain Res, 1017, 120-9.

Harte, M. and O'Connor, W. T. (2005) Evidence for a selective prefrontal cortical GABA(B) receptor-mediated inhibition of glutamate release in the ventral tegmental area: a dual probe microdialysis study in the awake rat. Neuroscience, 130, 215-22.

Heidbreder, C. A., Gardner, E. L., Xi, Z. X., Thanos, P. K., Mugnaini, M., Hagan, J. J. and Ashby, C. R., Jr. (2005) The role of central dopamine D3 receptors in drug addiction: a review of pharmacological evidence. Brain Res Brain Res Rev, 49, 77-105.

Henningfield, J. E., Stapleton, J. M., Benowitz, N. L., Grayson, R. F. and London, E. D. (1993) Higher levels of nicotine in arterial than in venous blood after cigarette smoking. Drug Alcohol Depend, 33, 23-9.

Houdi, A. A., Pierzchala, K., Marson, L., Palkovits, M. and Van Loon, G. R. (1991) Nicotine-induced alteration in Tyr-Gly-Gly and Met-enkephalin in discrete brain nuclei reflects altered enkephalin neuron activity. Peptides, 12, 161-6.

Hsieh, C. Y., Leslie, F. M. and Metherate, R. (2002) Nicotine exposure during a postnatal critical period alters NR2A and NR2B mRNA expression in rat auditory forebrain. Brain Res Dev Brain Res, 133, 19-25.

Hudmon, K. S., Kilfoy, B. A. and Prokhorov, A. V. (2006) The epidemiology of tobacco use and dependence. Crit Care Nurs Clin North Am, 18, 1-11, xi.

Huntley, G. W., Vickers, J. C. and Morrison, J. H. (1994) Cellular and synaptic localization of NMDA and non-NMDA receptor subunits in neocortex: organizational features related to cortical circuitry, function and disease. Trends Neurosci, 17, 536-43.

Hurley, K. M., Herbert, H., Moga, M. M. and Saper, C. B. (1991) Efferent projections of the infralimbic cortex of the rat. J Comp Neurol, 308, 249-76.

Hyman, S. E., Malenka, R. C. and Nestler, E. J. (2006) Neural mechanisms of addiction: the role of reward-related learning and memory. Annu Rev Neurosci, 29, 565-98.

Imperato, A., Mulas, A. and Di Chiara, G. (1986) Nicotine preferentially stimulates dopamine release in the limbic system of freely moving rats. Eur J Pharmacol, 132, 337-8. 
Jay, T. M., Burette, F. and Laroche, S. (1996) Plasticity of the hippocampal-prefrontal cortex synapses. $J$ Physiol Paris, 90, 361-6.

Jay, T. M., Glowinski, J. and Thierry, A. M. (1989) Selectivity of the hippocampal projection to the prelimbic area of the prefrontal cortex in the rat. Brain Res, 505, $337-40$.

Jayaram, P. and Steketee, J.D. (2004) Effects of repeated cocaine on medial prefrontal cortical GABAB receptor modulation of neurotransmission in the mesocorticolimbic dopamine system. J Neurochem, 90, 839-47.

Jedema, H. P. and Moghaddam, B. (1994) Glutamatergic control of dopamine release during stress in the rat prefrontal cortex. $J$ Neurochem, 63, 785-8.

Johnson, S. W. and North, R. A. (1992) Two types of neurone in the rat ventral tegmental area and their synaptic inputs. $J$ Physiol, 450, 455-68.

Johnson, S. W., Seutin, V. and North, R. A. (1992) Burst firing in dopamine neurons induced by N-methyl-D-aspartate: role of electrogenic sodium pump. Science, 258, 665-7.

Jose Lanca, A., Sanelli, T. R. and Corrigall, W. A. (2000) Nicotine-induced fos expression in the pedunculopontine mesencephalic tegmentum in the rat. Neuropharmacology, 39, 2808-17.

Kalivas, P. W. (2004) Recent understanding in the mechanisms of addiction. Curr Psychiatry Rep, 6, 347-51.

Kalivas, P. W., Churchill, L. and Klitenick, M. A. (1993) GABA and enkephalin projection from the nucleus accumbens and ventral pallidum to the ventral tegmental area. Neuroscience, 57, 1047-60.

Kalivas, P. W., Duffy, P. and Barrow, J. (1989) Regulation of the mesocorticolimbic dopamine system by glutamic acid receptor subtypes. J Pharmacol Exp Ther, 251, $378-87$.

Kalivas, P. W. and McFarland, K. (2003) Brain circuitry and the reinstatement of cocaine-seeking behavior. Psychopharmacology (Berl), 168, 44-56.

Katz, B. and Miledi, R. (1973) The effect of alpha-bungarotoxin on acetylcholine receptors. Br J Pharmacol, 49, 138-9.

Kenny, P. J. and Markou, A. (2001) Neurobiology of the nicotine withdrawal syndrome. Pharmacol Biochem Behav, 70, 531-49. 
Kirch, D. G., Gerhardt, G. A., Shelton, R. C., Freedman, R. and Wyatt, R. J. (1987) Effect of chronic nicotine administration on monoamine and monoamine metabolite concentrations in rat brain. Clin Neuropharmacol, 10, 376-83.

Klitenick, M. A., DeWitte, P. and Kalivas, P. W. (1992) Regulation of somatodendritic dopamine release in the ventral tegmental area by opioids and GABA: an in vivo microdialysis study. J Neurosci, 12, 2623-32.

Koob, G. F. (1992) Drugs of abuse: anatomy, pharmacology and function of reward pathways. Trends Pharmacol Sci, 13, 177-84.

Koop, C. E. and Luoto, J. (1982) "The Health Consequences of Smoking: Cancer," overview of a report of the Surgeon General. Public Health Rep, 97, 318-24.

Kosowski, A. R. and Liljequist, S. (2004) The NR2B-selective N-methyl-D-aspartate receptor antagonist Ro 25-6981 [(+/-)-( $\left.\mathrm{R}^{*}, \mathrm{~S}^{*}\right)$-alpha-(4-hydroxyphenyl)-betamethyl-4-(phenylmethyl)-1-pipe ridine propanol] potentiates the effect of nicotine on locomotor activity and dopamine release in the nucleus accumbens. $J$ Pharmacol Exp Ther, 311, 560-7.

Lambe, E. K., Picciotto, M. R. and Aghajanian, G. K. (2003) Nicotine induces glutamate release from thalamocortical terminals in prefrontal cortex. Neuropsychopharmacology, 28, 216-25.

Lanca, A. J., Adamson, K. L., Coen, K. M., Chow, B. L. and Corrigall, W. A. (2000) The pedunculopontine tegmental nucleus and the role of cholinergic neurons in nicotine self-administration in the rat: a correlative neuroanatomical and behavioral study. Neuroscience, 96, 735-42.

Laviolette, S. R. and van der Kooy, D. (2003) Blockade of mesolimbic dopamine transmission dramatically increases sensitivity to the rewarding effects of nicotine in the ventral tegmental area. Mol Psychiatry, 8, 50-9, 9.

Le Moal, M. and Simon, H. (1991) Mesocorticolimbic dopaminergic network: functional and regulatory roles. Physiol Rev, 71, 155-234.

Le Novere, N. and Changeux, J. P. (1995) Molecular evolution of the nicotinic acetylcholine receptor: an example of multigene family in excitable cells. $J \mathrm{Mol}$ Evol, 40, 155-72.

Li, S. P., Park, M. S., Kim, J. H. and Kim, M. O. (2004) Chronic nicotine and smoke treatment modulate dopaminergic activities in ventral tegmental area and nucleus accumbens and the gamma-aminobutyric acid type B receptor expression of the rat prefrontal cortex. J Neurosci Res, 78, 868-79. 
Li, Y., Hu, X. T., Berney, T. G., Vartanian, A. J., Stine, C. D., Wolf, M. E. and White, F. J. (1999) Both glutamate receptor antagonists and prefrontal cortex lesions prevent induction of cocaine sensitization and associated neuroadaptations. Synapse, 34, 169-80.

Li, Y., Vartanian, A. J., White, F. J., Xue, C. J. and Wolf, M. E. (1997) Effects of the AMPA receptor antagonist NBQX on the development and expression of behavioral sensitization to cocaine and amphetamine. Psychopharmacology (Berl), 134, 266-76.

Li, Y. and Wolf, M. E. (1997) Ibotenic acid lesions of prefrontal cortex do not prevent expression of behavioral sensitization to amphetamine. Behav Brain Res, 84, 2859.

Li, Y., Wolf, M. E. and White, F. J. (1999) The expression of cocaine sensitization is not prevented by MK-801 or ibotenic acid lesions of the medial prefrontal cortex. Behav Brain Res, 104, 119-25.

Licata, S. C., Schmidt, H. D. and Pierce, R. C. (2004) Suppressing calcium/calmodulindependent protein kinase II activity in the ventral tegmental area enhances the acute behavioural response to cocaine but attenuates the initiation of cocaineinduced behavioural sensitization in rats. Eur J Neurosci, 19, 405-14.

Liechti, M. E. and Markou, A. (2007) Interactive effects of the mGlu5 receptor antagonist MPEP and the mGlu2/3 receptor antagonist LY341495 on nicotine selfadministration and reward deficits associated with nicotine withdrawal in rats. Eur J Pharmacol, 554, 164-74.

Lindvall, O. and Bjorklund, A. (1978) Anatomy of the dopaminergic neuron systems in the rat brain. Adv Biochem Psychopharmacol, 19, 1-23.

Liu, X., Caggiula, A. R., Yee, S. K., Nobuta, H., Poland, R. E. and Pechnick, R. N. (2006) Reinstatement of nicotine-seeking behavior by drug-associated stimuli after extinction in rats. Psychopharmacology (Berl), 184, 417-25.

Lu, L., Grimm, J. W., Shaham, Y. and Hope, B. T. (2003) Molecular neuroadaptations in the accumbens and ventral tegmental area during the first 90 days of forced abstinence from cocaine self-administration in rats. J Neurochem, 85, 1604-13.

Mansvelder, H. D. and McGehee, D. S. (2000) Long-term potentiation of excitatory inputs to brain reward areas by nicotine. Neuron, 27, 349-57.

Mansvelder, H. D. and McGehee, D. S. (2002) Cellular and synaptic mechanisms of nicotine addiction. $J$ Neurobiol, 53, 606-17. 
McDonald, A. J. (1996) Glutamate and aspartate immunoreactive neurons of the rat basolateral amygdala: colocalization of excitatory amino acids and projections to the limbic circuit. J Comp Neurol, 365, 367-79.

McFarland, K., Davidge, S. B., Lapish, C. C. and Kalivas, P. W. (2004) Limbic and motor circuitry underlying footshock-induced reinstatement of cocaine-seeking behavior. J Neurosci, 24, 1551-60.

McFarland, K., Lapish, C. C. and Kalivas, P. W. (2003) Prefrontal glutamate release into the core of the nucleus accumbens mediates cocaine-induced reinstatement of drug-seeking behavior. J Neurosci, 23, 3531-7.

McGehee, D. S., Heath, M. J., Gelber, S., Devay, P. and Role, L. W. (1995) Nicotine enhancement of fast excitatory synaptic transmission in CNS by presynaptic receptors. Science, 269, 1692-6.

Meltzer, L. T., Christoffersen, C. L. and Serpa, K. A. (1997) Modulation of dopamine neuronal activity by glutamate receptor subtypes. Neurosci Biobehav Rev, 21, 511-8.

Moghaddam, B. (1993) Stress preferentially increases extraneuronal levels of excitatory amino acids in the prefrontal cortex: comparison to hippocampus and basal ganglia. J Neurochem, 60, 1650-7.

Mosner, A., Kuhlman, G., Roehm, C. and Vogel, W. H. (1997) Serotonergic receptors modify the voluntary intake of alcohol and morphine but not of cocaine and nicotine by rats. Pharmacology, 54, 186-92.

Murase, S., Grenhoff, J., Chouvet, G., Gonon, F. G. and Svensson, T. H. (1993) Prefrontal cortex regulates burst firing and transmitter release in rat mesolimbic dopamine neurons studied in vivo. Neurosci Lett, 157, 53-6.

Nakanishi, S., Nakajima, Y., Masu, M., Ueda, Y., Nakahara, K., Watanabe, D., Yamaguchi, S., Kawabata, S. and Okada, M. (1998) Glutamate receptors: brain function and signal transduction. Brain Res Brain Res Rev, 26, 230-5.

Nestler, E. J. (1992) Molecular mechanisms of drug addiction. $J$ Neurosci, 12, 2439-50.

Nestler, E. J., Terwilliger, R. Z., Walker, J. R., Sevarino, K. A. and Duman, R. S. (1990) Chronic cocaine treatment decreases levels of the $\mathrm{G}$ protein subunits Gi alpha and Go alpha in discrete regions of rat brain. J Neurochem, 55, 1079-82.

Nisell, M., Nomikos, G. G., Hertel, P., Panagis, G. and Svensson, T. H. (1996) Condition-independent sensitization of locomotor stimulation and mesocortical dopamine release following chronic nicotine treatment in the rat. Synapse, 22, 369-81. 
Nong, Y., Sorenson, E. M. and Chiappinelli, V. A. (1999) Fast excitatory nicotinic transmission in the chick lateral spiriform nucleus. J Neurosci, 19, 7804-11.

Oakman, S. A., Faris, P. L., Kerr, P. E., Cozzari, C. and Hartman, B. K. (1995) Distribution of pontomesencephalic cholinergic neurons projecting to substantia nigra differs significantly from those projecting to ventral tegmental area. $J$ Neurosci, 15, 5859-69.

Omelchenko, N. and Sesack, S. R. (2005) Laterodorsal tegmental projections to identified cell populations in the rat ventral tegmental area. J Comp Neurol, 483, 217-35.

Omelchenko, N. and Sesack, S. R. (2007) Glutamate synaptic inputs to ventral tegmental area neurons in the rat derive primarily from subcortical sources. Neuroscience, 146, $1259-74$.

Overton, P. G. and Clark, D. (1997) Burst firing in midbrain dopaminergic neurons. Brain Res Brain Res Rev, 25, 312-34.

Paquet, M., Tremblay, M., Soghomonian, J. J. and Smith, Y. (1997) AMPA and NMDA glutamate receptor subunits in midbrain dopaminergic neurons in the squirrel monkey: an immunohistochemical and in situ hybridization study. J Neurosci, 17, $1377-96$.

Parker, S. L., Fu, Y., McAllen, K., Luo, J., McIntosh, J. M., Lindstrom, J. M. and Sharp, B. M. (2004) Up-regulation of brain nicotinic acetylcholine receptors in the rat during long-term self-administration of nicotine: disproportionate increase of the alpha6 subunit. Mol Pharmacol, 65, 611-22.

Passafaro, M., Piech, V. and Sheng, M. (2001) Subunit-specific temporal and spatial patterns of AMPA receptor exocytosis in hippocampal neurons. Nat Neurosci, 4, $917-26$.

Paterson, N. E., Froestl, W. and Markou, A. (2004) The GABAB receptor agonists baclofen and CGP44532 decreased nicotine self-administration in the rat. Psychopharmacology (Berl), 172, 179-86.

Paterson, N. E., Froestl, W. and Markou, A. (2005) Repeated administration of the GABAB receptor agonist CGP44532 decreased nicotine self-administration, and acute administration decreased cue-induced reinstatement of nicotine-seeking in rats. Neuropsychopharmacology, 30, 119-28.

Paterson, N. E. and Markou, A. (2002) Increased GABA neurotransmission via administration of gamma-vinyl GABA decreased nicotine self-administration in the rat. Synapse, 44, 252-3. 
Paterson, N. E., Semenova, S., Gasparini, F. and Markou, A. (2003) The mGluR5 antagonist MPEP decreased nicotine self-administration in rats and mice. Psychopharmacology (Berl), 167, 257-64.

Paxinos, G. and Watson, C. (1986). The rat brain in stereotaxic coordinates. Academic Press, Sydney; Orlando.

Peto, R., Lopez, A. D., Boreham, J., Thun, M., Heath, C., Jr. and Doll, R. (1996) Mortality from smoking worldwide. Br Med Bull, 52, 12-21.

Picciotto, M. R. and Corrigall, W. A. (2002) Neuronal systems underlying behaviors related to nicotine addiction: neural circuits and molecular genetics. J Neurosci, 22, 3338-41.

Pidoplichko, V. I., DeBiasi, M., Williams, J. T. and Dani, J. A. (1997) Nicotine activates and desensitizes midbrain dopamine neurons. Nature, 390, 401-4.

Pierce, R. C., Reeder, D. C., Hicks, J., Morgan, Z. R. and Kalivas, P. W. (1998) Ibotenic acid lesions of the dorsal prefrontal cortex disrupt the expression of behavioral sensitization to cocaine. Neuroscience, 82, 1103-14.

Pierzchala, K., Houdi, A. A. and Van Loon, G. R. (1987) Nicotine-induced alterations in brain regional concentrations of native and cryptic Met- and Leu-enkephalin. Peptides, 8, 1035-43.

Pirot, S., Godbout, R., Mantz, J., Tassin, J. P., Glowinski, J. and Thierry, A. M. (1992) Inhibitory effects of ventral tegmental area stimulation on the activity of prefrontal cortical neurons: evidence for the involvement of both dopaminergic and GABAergic components. Neuroscience, 49, 857-65.

Pirot, S., Jay, T. M., Glowinski, J. and Thierry, A. M. (1994) Anatomical and electrophysiological evidence for an excitatory amino acid pathway from the thalamic mediodorsal nucleus to the prefrontal cortex in the rat. Eur J Neurosci, 6, 1225-34.

Pontieri, F. E., Tanda, G., Orzi, F. and Di Chiara, G. (1996) Effects of nicotine on the nucleus accumbens and similarity to those of addictive drugs. Nature, 382, 255-7.

Rahman, S., Zhang, J., Engleman, E. A. and Corrigall, W. A. (2004) Neuroadaptive changes in the mesoaccumbens dopamine system after chronic nicotine selfadministration: a microdialysis study. Neuroscience, 129, 415-24.

Redgrave, P., Prescott, T. J. and Gurney, K. (1999) Is the short-latency dopamine response too short to signal reward error? Trends Neurosci, 22, 146-51. 
Reid, M. S. and Berger, S. P. (1996) Evidence for sensitization of cocaine-induced nucleus accumbens glutamate release. Neuroreport, 7, 1325-9.

Risner, M. E. and Goldberg, S. R. (1983) A comparison of nicotine and cocaine selfadministration in the dog: fixed-ratio and progressive-ratio schedules of intravenous drug infusion. J Pharmacol Exp Ther, 224, 319-26.

Risso, F., Parodi, M., Grilli, M., Molfino, F., Raiteri, M. and Marchi, M. (2004) Chronic nicotine causes functional upregulation of ionotropic glutamate receptors mediating hippocampal noradrenaline and striatal dopamine release. Neurochem Int, 44, 293-301.

Robinson, T. E. and Kolb, B. (2004) Structural plasticity associated with exposure to drugs of abuse. Neuropharmacology, 47 Suppl 1, 33-46.

Roerig, B., Nelson, D. A. and Katz, L. C. (1997) Fast synaptic signaling by nicotinic acetylcholine and serotonin 5-HT3 receptors in developing visual cortex. $J$ Neurosci, 17, 8353-62.

Rose, J. E. and Corrigall, W. A. (1997) Nicotine self-administration in animals and humans: similarities and differences. Psychopharmacology (Berl), 130, 28-40.

Saal, D., Dong, Y., Bonci, A. and Malenka, R. C. (2003) Drugs of abuse and stress trigger a common synaptic adaptation in dopamine neurons. Neuron, 37, 577-82.

Sallette, J., Pons, S., Devillers-Thiery, A., Soudant, M., Prado de Carvalho, L., Changeux, J. P. and Corringer, P. J. (2005) Nicotine upregulates its own receptors through enhanced intracellular maturation. Neuron, 46, 595-607.

Sanghera, M. K., Trulson, M. E. and German, D. C. (1984) Electrophysiological properties of mouse dopamine neurons: in vivo and in vitro studies. Neuroscience, 12, 793-801.

Schilstrom, B., Fagerquist, M. V., Zhang, X., Hertel, P., Panagis, G., Nomikos, G. G. and Svensson, T. H. (2000) Putative role of presynaptic alpha $7 *$ nicotinic receptors in nicotine stimulated increases of extracellular levels of glutamate and aspartate in the ventral tegmental area. Synapse, 38, 375-83.

Schilstrom, B., Nomikos, G. G., Nisell, M., Hertel, P. and Svensson, T. H. (1998) Nmethyl-D-aspartate receptor antagonism in the ventral tegmental area diminishes the systemic nicotine-induced dopamine release in the nucleus accumbens. Neuroscience, 82, 781-9.

Schilstrom, B., Rawal, N., Mameli-Engvall, M., Nomikos, G. G. and Svensson, T. H. (2003) Dual effects of nicotine on dopamine neurons mediated by different nicotinic receptor subtypes. Int J Neuropsychopharmacol, 6, 1-11. 
Schultz, W., Dayan, P. and Montague, P. R. (1997) A neural substrate of prediction and reward. Science, 275, 1593-9.

Self, D. W. and Nestler, E. J. (1998) Relapse to drug-seeking: neural and molecular mechanisms. Drug Alcohol Depend, 51, 49-60.

Sesack, S. R. and Pickel, V. M. (1992) Prefrontal cortical efferents in the rat synapse on unlabeled neuronal targets of catecholamine terminals in the nucleus accumbens septi and on dopamine neurons in the ventral tegmental area. J Comp Neurol, 320, 145-60.

Sesack, S. R., Snyder, C. L. and Lewis, D. A. (1995) Axon terminals immunolabeled for dopamine or tyrosine hydroxylase synapse on GABA-immunoreactive dendrites in rat and monkey cortex. J Comp Neurol, 363, 264-80.

Seutin, V., Verbanck, P., Massotte, L. and Dresse, A. (1990) Evidence for the presence of $\mathrm{N}$-methyl-D-aspartate receptors in the ventral tegmental area of the rat: an electrophysiological in vitro study. Brain Res, 514, 147-50.

Shaham, Y., Shalev, U., Lu, L., De Wit, H. and Stewart, J. (2003) The reinstatement model of drug relapse: history, methodology and major findings. Psychopharmacology (Berl), 168, 3-20.

Shearman, E., Rossi, S., Sershen, H., Hashim, A. and Lajtha, A. (2005) Locally administered low nicotine-induced neurotransmitter changes in areas of cognitive function. Neurochem Res, 30, 1055-66.

Shi, S., Hayashi, Y., Esteban, J. A. and Malinow, R. (2001) Subunit-specific rules governing AMPA receptor trafficking to synapses in hippocampal pyramidal neurons. Cell, 105, 331-43.

Shippenberg, T. S. and Heidbreder, C. (1995) Sensitization to the conditioned rewarding effects of cocaine: pharmacological and temporal characteristics. $J$ Pharmacol Exp Ther, 273, 808-15.

Slotkin, T. A., Tate, C. A., Cousins, M. M. and Seidler, F. J. (2006) Prenatal nicotine exposure alters the responses to subsequent nicotine administration and withdrawal in adolescence: Serotonin receptors and cell signaling. Neuropsychopharmacology, 31, 2462-75.

Smith, S. S. and Fiore, M. C. (1999) The epidemiology of tobacco use, dependence, and cessation in the United States. Prim Care, 26, 433-61.

Spanagel, R. and Weiss, F. (1999) The dopamine hypothesis of reward: past and current status. Trends Neurosci, 22, 521-7. 
Steketee, J. D. (2003) Neurotransmitter systems of the medial prefrontal cortex: potential role in sensitization to psychostimulants. Brain Res Brain Res Rev, 41, 203-28.

Steketee, J. D. (2005) Cortical mechanisms of cocaine sensitization. Crit Rev Neurobiol, 17, 69-86.

Striplin, C. D. and Kalivas, P. W. (1992) Correlation between behavioral sensitization to cocaine and $G$ protein ADP-ribosylation in the ventral tegmental area. Brain Res, 579, 181-6.

Sugita, S., Johnson, S. W. and North, R. A. (1992) Synaptic inputs to GABAA and GABAB receptors originate from discrete afferent neurons. Neurosci Lett, 134, 207-11.

Summers, K. L. and Giacobini, E. (1995) Effects of local and repeated systemic administration of (-)nicotine on extracellular levels of acetylcholine, norepinephrine, dopamine, and serotonin in rat cortex. Neurochem Res, 20, 753-9.

Sun, W., Akins, C. K., Mattingly, A. E. and Rebec, G. V. (2005) Ionotropic glutamate receptors in the ventral tegmental area regulate cocaine-seeking behavior in rats. Neuropsychopharmacology, 30, 2073-81.

Sutton, M. A., Schmidt, E. F., Choi, K. H., Schad, C. A., Whisler, K., Simmons, D., Karanian, D. A., Monteggia, L. M., Neve, R. L. and Self, D. W. (2003) Extinction-induced upregulation in AMPA receptors reduces cocaine-seeking behaviour. Nature, 421, 70-5.

Svensson, T. H., Grenhoff, J. and Engberg, G. (1990) Effect of nicotine on dynamic function of brain catecholamine neurons. Ciba Found Symp, 152, 169-80; discussion 180-5.

Sziraki, I., Sershen, H., Benuck, M., Hashim, A. and Lajtha, A. (1998) Receptor systems participating in nicotine-specific effects. Neurochem Int, 33, 445-57.

Taber, M. T., Das, S. and Fibiger, H. C. (1995) Cortical regulation of subcortical dopamine release: mediation via the ventral tegmental area. $J$ Neurochem, $\mathbf{6 5}$, 1407-10.

Taber, M. T. and Fibiger, H. C. (1995) Electrical stimulation of the prefrontal cortex increases dopamine release in the nucleus accumbens of the rat: modulation by metabotropic glutamate receptors. $J$ Neurosci, 15, 3896-904.

Takahata, R. and Moghaddam, B. (1998) Glutamatergic regulation of basal and stimulusactivated dopamine release in the prefrontal cortex. $J$ Neurochem, 71, 1443-9. 
Takahata, R. and Moghaddam, B. (2000) Target-specific glutamatergic regulation of dopamine neurons in the ventral tegmental area. J Neurochem, 75, 1775-8.

Tempel, A. and Zukin, R. S. (1987) Neuroanatomical patterns of the mu, delta, and kappa opioid receptors of rat brain as determined by quantitative in vitro autoradiography. Proc Natl Acad Sci U S A, 84, 4308-12.

Tessari, M., Pilla, M., Andreoli, M., Hutcheson, D. M. and Heidbreder, C. A. (2004) Antagonism at metabotropic glutamate 5 receptors inhibits nicotine- and cocainetaking behaviours and prevents nicotine-triggered relapse to nicotine-seeking. Eur J Pharmacol, 499, 121-33.

Thierry, A. M., Blanc, G., Sobel, A., Stinus, L. and Golwinski, J. (1973) Dopaminergic terminals in the rat cortex. Science, 182, 499-501.

Thierry, A. M., Gioanni, Y., Degenetais, E. and Glowinski, J. (2000) Hippocampoprefrontal cortex pathway: anatomical and electrophysiological characteristics. Hippocampus, 10, 411-9.

Toda, S., Shen, H. W., Peters, J., Cagle, S. and Kalivas, P. W. (2006) Cocaine increases actin cycling: effects in the reinstatement model of drug seeking. J Neurosci, 26, 1579-87.

Tong, Z. Y., Overton, P. G. and Clark, D. (1996) Stimulation of the prefrontal cortex in the rat induces patterns of activity in midbrain dopaminergic neurons which resemble natural burst events. Synapse, 22, 195-208.

Tzschentke, T. M. (2001) Pharmacology and behavioral pharmacology of the mesocortical dopamine system. Prog Neurobiol, 63, 241-320.

Tzschentke, T. M. and Schmidt, W. J. (1999) Functional heterogeneity of the rat medial prefrontal cortex: effects of discrete subarea-specific lesions on drug-induced conditioned place preference and behavioural sensitization. Eur J Neurosci, 11, 4099-109.

Tzschentke, T. M. and Schmidt, W. J. (2000) Differential effects of discrete subareaspecific lesions of the rat medial prefrontal cortex on amphetamine- and cocaineinduced behavioural sensitization. Cereb Cortex, 10, 488-98.

Tzschentke, T. M. and Schmidt, W. J. (2000) Differential effects of quinolinic acid lesions of the medial prefrontal cortex on the expression of morphine- and dizocilpine- induced behavioural plasticity in the rat. Neurosci Lett, 283, 125-8.

Tzschentke, T. M. and Schmidt, W. J. (2000) Functional relationship among medial prefrontal cortex, nucleus accumbens, and ventral tegmental area in locomotion and reward. Crit Rev Neurobiol, 14, 131-42. 
Valentine, J. D., Hokanson, J. S., Matta, S. G. and Sharp, B. M. (1997) Selfadministration in rats allowed unlimited access to nicotine. Psychopharmacology (Berl), 133, 300-4.

Van Bockstaele, E. J. and Pickel, V. M. (1995) GABA-containing neurons in the ventral tegmental area project to the nucleus accumbens in rat brain. Brain Res, 682, 215 21.

Vezina, P. (1996) D1 dopamine receptor activation is necessary for the induction of sensitization by amphetamine in the ventral tegmental area. J Neurosci, 16, 241120.

Walaas, I. and Fonnum, F. (1979) The distribution and origin of glutamate decarboxylase and choline acetyltransferase in ventral pallidum and other basal forebrain regions. Brain Res, 177, 325-36.

Wang, B., Shaham, Y., Zitzman, D., Azari, S., Wise, R. A. and You, Z. B. (2005) Cocaine experience establishes control of midbrain glutamate and dopamine by corticotropin-releasing factor: a role in stress-induced relapse to drug seeking. $J$ Neurosci, 25, 5389-96.

Wang, F., Chen, H., Steketee, J. D. and Sharp, B. M. (2007) Upregulation of ionotropic glutamate receptor subunits within specific mesocorticolimbic regions during chronic nicotine self-administration. Neuropsychopharmacology, 32, 103-9.

Wang, T. and French, E. D. (1993) Electrophysiological evidence for the existence of NMDA and non-NMDA receptors on rat ventral tegmental dopamine neurons. Synapse, 13, 270-7.

Wang, T. and French, E. D. (1993) L-glutamate excitation of A10 dopamine neurons is preferentially mediated by activation of NMDA receptors: extra- and intracellular electrophysiological studies in brain slices. Brain Res, 627, 299-306.

Wang, T., O'Connor, W. T., Ungerstedt, U. and French, E. D. (1994) N-methyl-Daspartic acid biphasically regulates the biochemical and electrophysiological response of A10 dopamine neurons in the ventral tegmental area: in vivo microdialysis and in vitro electrophysiological studies. Brain Res, 666, 255-62.

Ward, J. M., Cockcroft, V. B., Lunt, G. G., Smillie, F. S. and Wonnacott, S. (1990) Methyllycaconitine: a selective probe for neuronal alpha-bungarotoxin binding sites. FEBS Lett, 270, 45-8.

Watkins, S. S., Koob, G. F. and Markou, A. (2000) Neural mechanisms underlying nicotine addiction: acute positive reinforcement and withdrawal. Nicotine Tob Res, 2, 19-37. 
Westerink, B. H., Kwint, H. F. and deVries, J. B. (1996) The pharmacology of mesolimbic dopamine neurons: a dual-probe microdialysis study in the ventral tegmental area and nucleus accumbens of the rat brain. $J$ Neurosci, 16, 2605-11.

White, F. J., Hu, X. T., Zhang, X. F. and Wolf, M. E. (1995) Repeated administration of cocaine or amphetamine alters neuronal responses to glutamate in the mesoaccumbens dopamine system. J Pharmacol Exp Ther, 273, 445-54.

Wise, R. A. (1996) Addictive drugs and brain stimulation reward. Annu Rev Neurosci, 19, 319-40.

Wise, R. A. (2006) Role of brain dopamine in food reward and reinforcement. Philos Trans $R$ Soc Lond B Biol Sci, 361, 1149-58.

Wolf, M. E., Dahlin, S. L., Hu, X. T., Xue, C. J. and White, K. (1995) Effects of lesions of prefrontal cortex, amygdala, or fornix on behavioral sensitization to amphetamine: comparison with N-methyl-D-aspartate antagonists. Neuroscience, 69, 417-39.

Wolf, M. E., Sun, X., Mangiavacchi, S. and Chao, S. Z. (2004) Psychomotor stimulants and neuronal plasticity. Neuropharmacology, 47 Suppl 1, 61-79.

Xi, Z. X. and Stein, E. A. (2002) Blockade of ionotropic glutamatergic transmission in the ventral tegmental area reduces heroin reinforcement in rat. Psychopharmacology (Berl), 164, 144-50.

Yamaguchi, T., Sheen, W. and Morales, M. (2007) Glutamatergic neurons are present in the rat ventral tegmental area. Eur J Neurosci, 25, 106-18.

Yamazaki, Y., Fujii, S., Jia, Y. and Sumikawa, K. (2006) Nicotine withdrawal suppresses nicotinic modulation of long-term potentiation induction in the hippocampal CA1 region. Eur J Neurosci, 24, 2903-16.

Zhang, X. F., Hu, X. T., White, F. J. and Wolf, M. E. (1997) Increased responsiveness of ventral tegmental area dopamine neurons to glutamate after repeated administration of cocaine or amphetamine is transient and selectively involves AMPA receptors. J Pharmacol Exp Ther, 281, 699-706.

Zhao, R., Chen, H. and Sharp, B. M. (2007) Nicotine-Induced Norepinephrine Release in Hypothalamic Paraventricular Nucleus and Amygdala Is Mediated by N-MethylD-aspartate Receptors and Nitric Oxide in the Nucleus Tractus Solitarius. $J$ Pharmacol Exp Ther, 320, 837-44. 


\section{Vita}

Fan Wang was born in Nanjing, China, on Dec 28, 1978, the son of Yongbo Wang and Jianping Li. He attended Nanjing No.9 High School, Nanjing, China, in 1994 and graduated in 1997. He entered China Pharmaceutical University in Nanjing, China, in September 1997 and completed Bachelor of Engineering degree in Biopharmacy. In August of 2001, he was admitted into University of Tennessee Health Science Center for his Ph.D. degree in Pharmacology. 